\title{
Impacts of Double-diffusive Processes on the Thermohaline Circulation
}

by

\author{
Jubao Zhang
}

B.S. University of Science and Technology of China, Hefei, China, (1990)

M.S. Inst. of Atmos. Phys., Chinese Academy of Sciences, Beijing, China, (1993)

Submitted in partial fulfillment of the

requirements for the degree of

$a$

Doctor of Philosophy

at the

MASSACHUSETTS INSTITUTE OF TECHNOLOGY

\begin{tabular}{|c|c|}
\hline 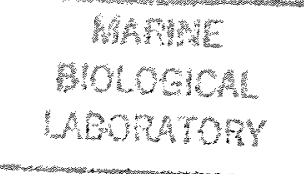 & WOODS HOLE OCEANOGRAPHIC INSTITUTION \\
\hline LIDRARY & August 1998 \\
\hline $\begin{array}{l}\text { WOODS HOLE, WhSS } \\
\text { W. H. O }\end{array}$ & (c) Jubao Zhang 1998 \\
\hline
\end{tabular}

Signature of Author

Joint Program in Physical Oceanography Massachusetts Institute of Technology

Woods Hole Oceanographic Institution August, 1998

Certified by

Raymond W. Schmitt

Senior Scientist

Thesis Supervisor

Accepted by

W. Brechner Owens

Chairman, Joint Committee for Physical Oceanography Massachusetts Institute of Technology Woods Hole Oceanographic Institution 


\title{
Impacts of Double-diffusive Processes on the Thermohaline Circulation by \\ Jubao Zhang
}

\author{
Submitted in partial fulfillment of the requirements for the degree of \\ Doctor of Philosophy at the Massachusetts Institute of Technology \\ and the Woods Hole Oceanographic Institution
}

August 7, 1998

\begin{abstract}
Double-diffusive processes are studied and parameterized, and their impacts on the oceanic thermohaline circulation are investigated by single-hemisphere numerical models and scaling analysis.

Scaling analysis on the thermohaline circulation has been done under three types of surface boundary conditions. (a) Under "relaxation" conditions, there is a two-thirds power law dependence of the meridional overturning rate (and the poleward heat transport) on the diapycnal diffusivity. For any given external forcing, there is only one equilibrium state for the thermohaline circulation. (b) Under "flux" boundary conditions, there is a half power law dependence of the meridional overturning rate on the diapycnal diffusivity. Only one mode is possible for given external forcing. (c) Under "mixed" boundary conditions, multiple equilibria become possible. For given thermal forcing, the existence of multiple equilibria depends on the relative contributions of diapycnal diffusivity and the hydrologic forcing. Numerical experiments are implemented to test the above scaling arguments. Consistent results have been obtained under the above three types of boundary conditions. These provide a basis for understanding how the thermohaline circulation depends on the diapycnal diffusivity, which we know is influenced by the double-diffusive processes of "salt fingering" and "diffusive layering" in some parts of the ocean.

In order to examine this issue, the double-diffusive processes are parameterized by diapycnal eddy diffusivities for heat and salt that are different and depend on the local density ratio, $R_{p}=\alpha T_{z} / \beta S_{z}$. A background diffusivity is applied to represent turbulent mixing in the stratified environment. The implementation of this double-diffusive parameterization in numerical models has significant impacts on the thermohaline circulation. (a) Under "relaxation" boundary conditions, the meridional overturning rate and the poleward heat transport are reduced, and water mass properties are also changed. Similar results are obtained under "flux" boundary conditions. (b) Under "mixed" boundary conditions, the critical freshwater flux for the existence of the thermal mode becomes smaller with the double-diffusive parameterization. The extent to which the thermohaline circulation is affected by double-diffusive processes depends on the magnitude of the freshwater forcing.
\end{abstract}

Thesis Supervisor: Raymond W. Schmitt, Title: Senior Scientist, Woods Hole Oceanographic Institution 


\section{Dedicated to}

\section{my parents, brothers and sisters}




\section{Acknowledgments}

First of all I would like to thank my advisor Ray Schmitt for his patient guidance and optimistic encouragement. Five years ago when I first arrived in Woods Hole, Ray led me to the beach, and on the road he showed me how to distinguish poison ivy from wild berry leaves. In the later years he taught me how to avoid the poisonous stuff in my thesis work. His deep insight into a wide range of scientific problems and his physical intuition have motivated and helped me in both scaling analysis and numerical work. Every time I submitted a report or a draft, Ray read it very carefully and then my poorly written stuff was much polished within a week. Also, I would like to thank him for many valuable, constructive, and joyful discussions, and his fatherhood experience. I have been fortunate to have the opportunity to work with Ray for the past five years.

I want to express my sincere gratitude to Rui Xin Huang, a critical member on my thesis committee. Xin introduced the thesis topic to me and became a collaborator ever since. His suggestions, criticisms, and comments on numerical models and the thermohaline circulation helped a lot. He generously allowed me to use his most updated SGI work station. Also, his wisdom, experience, friendship and competition on the Mahjong table made my study here not only educating but also entertaining. I would also like to thank other committee members John Toole, Jochem Marotzke and Glenn Flierl for their critical comments and suggestions. John read my draft thesis and two previous papers very carefully and greatly improved the writing. He also inspired me into checking the sensitivity of the thermohaline circulation to the Coriolis parameter which turned out to be a very interesting problem. I had many motivating discussions with Jochem at MIT, and Glenn always came up with a very satisfying answer whenever I had a question for him. Thanks to Mike Spall for serving as the chair of my defense. One significant memory in the Joint Program was the class with the master in lecturing, Joe Pedlosky. Joe also helped my thesis in many ways, and he has always been friendly and patient. Discussions with Carl Wunsch and John Marshall have been very helpful, even though John Marshall was not convinced of the importance of double diffusion. Ellyn Montgomery helped to guarantee the computer resources I needed. I am indebted to GFDL of Princeton University for providing different versions of ocean models, and especially Ron Pacanowski for helping to set up the model. 
I am grateful to my classmates and friends in the Joint Program and MIT. The teamwork in the courses and preparation for general exams, with my classmates Richard Wardle, Sandra Werner, Melissa Bowen and Dan Goldner, was a sweet memory, as well as their help and patience in my language development. Thanks to Richard for sharing support for Michael Jordan's Bulls and the New England Patriots, as well as our joy in France World Cup'98. Thanks to Sandra for her sincere friendship. Thanks to Misha Chechelnitsky for sharing his experience in resume writing. I would also like to thank Lous St. Laurent for providing me the NATRE data plot for my defense talk, and Brian Arbic for many pleasant discussions, and Jay Austin and Jamie Pringle for sharing the style files for the thesis writing. Shi Jiang has always been a good talker and listener over a wide range of topics for the past four years, from oceanography, meteorology to Wall street and Mahjong. I am grateful to Huai-Min Zhang, Wenjie $\mathrm{Hu}$, Min Jiang, Dezhang Chu, Xiaoou Tang, Zheng Liu for their help when I first entered the Joint Program. Lisan Yu, Bing Ni, Zhou Yu, Tony Lee, Xiaoli Wang have all been very helpful in my stay at MIT. I also thank my officemates Richard, Natasha and Rong for all the nice conversations and comfortable environment in room 54-1419.

In Boston and the Falmouth area I have many wonderful friends, among whom are Xiaohui Luo, Luping Zou, Fen Zhang, Minghui Zhang, Wei Hu, Xiangze Jin and Jiarui Dong. Their friendship and help made my life a lot easier and they were always there when I needed some help. Also, I am indebted to my host family, Bill Long and Judy Long, for introducing and sharing the American culture and hospitality, and many nice dinners.

I sincerely thank my wife, Yang Gao, for all her love, support, patience, understanding, sacrifice and strength. She should be rewarded a degree of PhT (Push husband Through). I also thank my son Ryan for all the joy and pride he brought to me. Most of all, my family have always been my spiritual spine over so many years, their love, care and courage have always accompanied me. It would be impossible for me to reach this far in academics without the selfless support of my parents, brothers and sisters.

This thesis is supported by a grant from the Ocean Sciences Division of the National Science Foundation, OCE94-15589. 


\section{Contents}

$\begin{array}{ll}\text { Abstract } & 2\end{array}$

$\begin{array}{ll}\text { Acknowledgments } & 4\end{array}$

1 Introduction $\quad 10$

1.1 Thermohaline Circulation and Climate . . . . . . . . . . 10

1.2 Double Diffusion in the Oceans . . . . . . . . . . . . . . . 11

1.3 Motivation ...................... 15

1.3.1 Scaling Analysis of Thermohaline Circulation . . . . . . 15

1.3.2 Double Diffusion and Thermohaline Circulation . . . . . . . 17

1.4 Overview . . . . . . . . . . . . . . . . . . . . 18

2 Scaling Analysis and Numerical Experiments on the Thermohaline $\begin{array}{ll}\text { Circulation } & 20\end{array}$

2.1 Introduction . . . . . . . . . . . . . . . . 20

2.2 Scaling Analysis . . . . . . . . . . . . . . . . . 22

2.2 .1 Equations ..................... 22

2.2.2 Relaxation Boundary Conditions . . . . . . . . . . . . 24 
2.2.3 Flux Boundary Conditions . . . . . . . . . . . 25

2.2.4 Mixed Boundary Conditions . . . . . . . . . . . . . 29

2.3 Description of the Numerical Model . . . . . . . . . . . . . . . . . . . 41

2.4 Numerical Experiments . . . . . . . . . . . . . . . . 45

2.4.1 General Sensitivity Experiments . . . . . . . . . . . . 45

2.4.2 Relaxation Boundary Conditions . . . . . . . . . . . . . 48

2.4.3 Flux Boundary Conditions . . . . . . . . . . . 53

2.4.4 Mixed Boundary Conditions . . . . . . . . . . 57

2.5 Discussion and Conclusions . . . . . . . . . . . . . . 67

3 Parameterization of Double-diffusive Processes and Scaling Analysis 69

3.1 Introduction . . . . . . . . . . . . . . . . . . 69

3.2 Parameterization of Double Diffusion . . . . . . . . . . . 70

3.2.1 Parameterization of Salt Fingering . . . . . . . . . 70

3.2.2 Parameterization of Diffusive Layering . . . . . . . . . . . . . 72

3.2.3 Non-double-diffusive Process . . . . . . . . . . . . . . 73

3.3 Scaling Analysis of the Impacts of Double Diffusion on the Thermohaline Circulation . . . . . . . . . . . . . . . 73

3.3.1 Relaxation Boundary Conditions . . . . . . . . . 76

3.3.2 Flux Boundary Conditions . . . . . . . . . . . . . . 81

3.3.3 Mixed Boundary Conditions . . . . . . . . . . . . . 82

3.4 Summary .............................. 85

4 Impacts of Double-diffusive Processes on Thermohaline Circulation $\begin{array}{ll}\text { under Restoring Boundary Conditions } & 87\end{array}$ 
4.1 Introduction . . . . . . . . . . . . . . . . . 87

4.2 Control Experiments . . . . . . . . . . . . . . . . . . 90

4.2 .1 The Numerical Experiments . . . . . . . . . . . . . 99 90

4.2.2 Diffusivity Variability in Run DDP . . . . . . . . . . . 91

4.2 .3 Meridional Overturning . . . . . . . . . . . . 93

4.2.4 Poleward Heat Transport . . . . . . . . . . . . . . 97

4.2 .5 Turner Angle . . . . . . . . . . . . . . . . . . . . . 99

4.2.6 Temperature and Salinity Distribution _. . . . . . . 102

4.3 Sensitivity Experiments _ . . . . . . . . . . . . . . 105

4.3.1 Parameter Sensitivity . . . . . . . . . . . . . . . 105

4.3.2 Sensitivity to Restraint $T_{z, c} \ldots \ldots \ldots \ldots \ldots$

4.3.3 Salt Fingering and Diffusive Layering Alone . . . . . . . 108

4.3.4 Sensitivity to the Vertical Resolution . . . . . . . . . . . 109

4.4 Summary . . . . . . . . . . . . . . . . . . 110

5 Impacts of Double-diffusive Processes on Thermohaline Circulation under Mixed Boundary Conditions 113

5.1 Introduction . . . . . . . . . . . . . . 113

5.2 Model Configuration . . . . . . . . . . . . . . . . . . . . 114

5.3 Quasi-Equilibrium Experiments _. . . . . . . . . . . . . 117

5.3.1 Two Control Experiments . . . . . . . . . . . . . 117

5.3.2 Parameter Sensitivity Experiments _. . . . . . . 120

5.4 Individual Experiments . . . . . . . . . . . . . . . . . 122

5.4.1 Weak Freshwater Forcing . . . . . . . . . . . . . . 122 
5.4.2 Intermediate Freshwater Forcing . . . . . . . . . . . . . 128

5.4 .3 Strong Freshwater Forcing . . . . . . . . . . . . . . . 133

5.5 Summary .............................. 137

6 Conclusions $\quad 139$

6.1 Summary . . . . . . . . . . . . . . . . . . . . 139

6.2 Future Work . . . . . . . . . . . . . . . . . . . . . 141

A Solution of the equation $R^{4}=F|R-1|$

A.1 Thermal Mode(s) Solution . . . . . . . . . . . . . . 143

A.2 Haline Mode(s) Solution . . . . . . . . . . . . . . 148

$\begin{array}{ll}\text { References } & 150\end{array}$ 


\section{Chapter 1}

\section{Introduction}

\subsection{Thermohaline Circulation and Climate}

Scientific, economic and even political interest in potential global climate changes and their impact on our environment have been increasing. The ocean is involved in the climate system primarily because it stores heat, water and carbon dioxide, moves them around on the earth, and exchanges these and other elements with the atmosphere. The search for an understanding of climate change, both past and present, has led directly to the ocean and especially to the ocean's thermohaline circulation. Variations in the stability or variability properties of the ocean's thermohaline circulation and hence its associated poleward transport of heat would have significant impact on both local and global climate. The ocean, with its large thermal stability and its potential to store both anthropogenic and natural greenhouse gases, serves as an important regulator of climate.

Data for the deep ocean are very sparse, reflecting the difficulty and expense of obtaining them. On the other hand, with the advent of a new generation of supercomputers, ocean general circulation models (OGCM) have become increasingly important tools to study the physics of the thermohaline circulation.

The thermohaline circulation is driven by heat and freshwater fluxes at the sea surface, thus the upper boundary conditions for temperature and salinity are very important. 
There are three types of upper boundary conditions in common use: (i) "relaxation" boundary conditions, i.e., both sea surface temperature (SST) and sea surface salinity (SSS) are restored to some prescribed values; (ii) "mixed" boundary conditions, i.e., SST is relaxed to the prescribed values and a virtual salt flux condition is used for SSS; and (iii) "flux" boundary conditions for both temperature and salinity. Previous work showed that the thermohaline circulation behaves qualitatively differently under these various upper boundary conditions. For example, under mixed boundary conditions, multiple equilibria can be obtained (Stommel, 1960; F. Bryan, 1986; Marotzke, 1990; Weaver and Hughes, 1992), and the existence of multiple equilibria depends on the magnitude and distribution of surface freshwater forcing.

The strength of the meridional overturning circulation (MOC) is directly related to the magnitude of the poleward heat transport and is thus of central concern to climate studies. The MOC rate is intimately bound up with the rate at which the ocean mixes in the vertical (or diapycnal) direction. F. Bryan (1987) found that the MOC in his general circulation model appeared to be proportional to $K^{1 / 3}$ under relaxation boundary conditions, where $K$ is the vertical diffusivity of density.

It has long been assumed that salt and heat are mixed at the same diapycnal rate in OGCMs. However, laboratory experiments and observations of double-diffusive processes in the ocean suggest that there is a significant difference in the rates at which salt and heat are mixed diapycnally. Model results indicate that this has a significant consequence for the thermohaline circulation. Gargett and Holloway (1992) assumed that $K_{T} \neq K_{S}$ as would be expected in mixing processes like double diffusion (where $K_{T}$ and $K_{S}$ are the vertical diffusivities of temperature and salinity respectively), and they found significant changes in the structure and strength of the thermohaline circulation. This thesis is aimed at understanding how a more accurate parameterization of double-diffusive processes can affect the thermohaline circulation, as well as exploring the influence of different upper boundary conditions in models.

\subsection{Double Diffusion in the Oceans}

The modern study of double-diffusive convection began with Melvin Stern's article on "The Salt Fountain and Thermohaline Convection" in 1960. In that paper, he showed 
how opposing stratifications of two component species could drive convection if their diffusivities differed. Reviews of double diffusion can be found in Turner (1973) and Schmitt (1994). There are two types of double-diffusive convection in the oceans:

(i) Salt fingers can form when warmer saltier water overlies colder fresher water, such that

$$
T_{z}>0, S_{z}>0,1<R_{\rho}<k_{t} / k_{s}
$$

where $R_{\rho}=\alpha T_{z} / \beta S_{z}$ is the density ratio, $k_{t}$ and $k_{s}$ are the molecular diffusivities of heat and salt respectively. The ratio of the heat and salt molecular diffusivities is about 100 , so even a very weak destabilizing salinity gradient can induce salt fingering. The instability appears as narrow column of up and down going fluid, exchanging heat laterally and allowing the salt to fall out.

(ii) When colder fresher water overlies warmer saltier water, with

$$
T_{z}<0, S_{z}<0,1>R_{\rho}>k_{s} / k_{t}
$$

diffusive layering occurs. In this case, heating from below drives convection in layers (similar to Rayleigh-Benard) and the salt stratification maintains the stability of thin, diffusive interfaces.

Conditions favorable for double diffusion are very common in the oceans. In the subtropics the ocean gains heat from the sun, and evaporation exceeds precipitation. Thus, in comparison with the deep ocean, the upper ocean is warm and salty; a stratification which favors salt fingering. Ingham (1966) reports that $90 \%$ of the main thermocline of the Atlantic Ocean has a density ratio less than 2.3. Schmitt (1990) finds that $95 \%$ of the upper kilometer in the Atlantic at $24^{\circ} \mathrm{N}$ is salt fingering favorable. In the western tropical North Atlantic an area of over 1 million $\mathrm{km}^{2}$ was found to contain strong thermohaline staircases in the main thermocline (Schmitt et al., 1987). Systematic, large-scale water mass changes within the staircase layers were observed, which cannot be explained by conventional mechanical turbulence, but fit nicely the enhanced salt flux expected from salt fingers. This location is characterized by a strong vertical salt gradient and a low value of the density ratio $R_{\rho}$. Microstructure observations (Gregg and Sanford, 1987; Lueck, 1987; Marmorino et al., 1987) revealed narrow band, limited amplitude structures of the predicted scale for salt fingers within the interfaces, and plume-like, convective structures within the 
mixed layers. The intensity of the microstructure indicates that an eddy diffusivity for salt of 1-2 $\mathrm{cm}^{2} \mathrm{~s}^{-1}$ must apply within the staircase (Schmitt, 1988; Kunze, 1990). While lower than originally expected, this is quite substantial compared to the weak background value of $0.07 \mathrm{~cm}^{2} \mathrm{~s}^{-1}$ predicted for the canonical Garret and Munk (1976) internal wave field (Gregg, 1989). Indeed, the vertical heat flux derived from the microstructure measurements is nearly $3 \mathrm{Wm}^{-2}$, which exceeds the flux estimated in a strongly turbulent, but weakly stratified, abyssal site near the Mid-Atlantic Ridge (Polzin et al., 1997). The salt finger mixing in this region of the Atlantic is thought to be responsible for the water mass conversion observed in the thermocline waters transiting from the South Atlantic to the Gulf Stream in the Florida Straits (Schmitz et al., 1993).

In addition to the strong vertical mixing inferred for the staircase regions such as the tropical Atlantic, Mediterranean Outflow and the Tyrhennian Sea, where the density ratio is less than 1.7, fingers are also thought to play some role in mixing in the broad central waters of the world ocean, where the density ratio is only slightly higher. Some contribution is very likely, since turbulence due to shear instability is infrequent, occurring only a few percent of the time (Polzin, 1996). Laboratory experiments have shown that fingers grow rapidly after a turbulent mixing event, when the density ratio is near the common oceanic value of 2 (Taylor, 1991). When fingers alone exist, there is a strong increase in laboratory fluxes as the density ratio drops below 2.0 (McDougall and Taylor, 1984). Gargett and Schmitt (1982) find microscale signature of fingers in the Central Waters of the North Pacific. Mack $(1985,1989)$ and Mack and Schoeberlein (1993) find salt finger signatures in microstructure data from the upper thermocline of the Sargasso Sea, when the local density ratio falls below 3.0. Marmorino (1987) also finds the distinct spectral signatures of salt fingers in data from the seasonal thermocline of the Sargasso Sea. Recent theoretical work of Shen and Schmitt (1995) provides a salt finger model spectrum which is in good agreement with the observed microstructure. It is based on the "dispersion relation" for salt fingers derived by Schmitt (1979) which shows that finger growth times are comparable to the local buoyancy period only when $R_{\rho}$ is less than 2 . Hamilton et al. (1989) report on salt finger signatures in microstructure data from the main thermocline of the eastern North Atlantic which appear to provide a significant vertical nutrient flux. Recently, St. Laurent and Schmitt (1998) examined data from the North Atlantic 
Tracer Release Experiment, and found that $K_{T}=(0.08 \pm 0.01) \mathrm{cm}^{2} \mathrm{~s}^{-1}$ and $K_{S}=$ $(0.13 \pm 0.01) \mathrm{cm}^{2} s^{-1}$ for the neutral surface at about $300 \mathrm{~m}$, where $K_{T}, K_{S}$ are the vertical eddy diffusivities for temperature and salinity respectively.

Due to surface cooling and excess precipitation, the surface water in polar and subpolar regions is colder and fresher than the subsurface water. As a result, the other type of double diffusion, diffusive convection (or layering), is possible. In this case, the heat content is the destabilizing component and more heat is mixed upward than salt. Though the initial process may appear as an oscillatory overstability (Stern, 1960; Shirtcliffe, 1967), the fully developed state is one of alternating convecting layers and high gradient sheets (Turner, 1973); hence the name. The characteristic staircase layers have long been observed beneath the ice in the Arctic Ocean (Neal et al., 1969; Neshyba et al., 1971). Padman and Dillon (1987, 1988) provide evidence for widespread diffusive staircases in both the Beaufort Sea and Canadian Basin. The Antarctic contains sites of strong diffusive layering as well. Muench et al. (1990) found numerous thermohaline staircases in the northwestern Weddell Sea during March 1986. Throughout an approximately $40,000 \mathrm{~km}^{2}$ study area, they observed that between 100 and $180 \mathrm{~m}$ depth, staircases with a typical scale of 1-5 m occurred almost everywhere. Between 180-500 m depth, staircases were observed over $\sim 50 \%$ of the study area, with layer thicknesses of $10-100 \mathrm{~m}$. Vertical heat fluxes of up to $15 \mathrm{Wm}^{-2}$ were estimated, which is significant in the upper ocean heat budget and may help maintain ice-free conditions. Robertson et al. (1995) also found that diapycnal fluxes in the Weddell Sea were dominated by diffusive convection. Similarly, Martinson (1990) found it to be important in a model of winter sea ice formation. Kelley $(1984,1990)$ has provided a parameterization of the mixing due to diffusive convection which derives from a successful scaling of the layer thicknesses in both the laboratory and ocean. Effective diffusivities are weaker than in the salt finger regime, consistent with differences between convective transports realized with fingers and conductive fluxes that characterize diffusive interfaces. 


\subsection{Motivation}

\subsubsection{Scaling Analysis of Thermohaline Circulation}

Stommel (1961) first recognized the possibility of multiple solutions of the thermohaline circulation in an idealized 2-box model. The sudden change associated with transition from one state of the thermohaline circulation to the other might be associated with catastrophic change in climate; thus, the thermohaline circulation has become an important topic during the past decade. Marotzke (1990) used a 2-box model similar to Stommel (1961) to investigate the dependence of the existence of multiple solutions upon the ratio of salt flux forcing versus thermal forcing; he found that there is an upper limit to the equivalent salt flux, above which only one thermal mode can exist. Similar results have been obtained in the $2 \times 2$ box model of Huang et al. (1992).

Box models provide a simple and elegant way to study the multiple equilibria of the thermohaline circulation under mixed boundary conditions (see review of Whitehead, 1995). However, these models make a strong assumption that the meridional velocity is proportional to the north-south density difference, whose linear proportionality coefficient can not be determined in connection with the real ocean or the parameters in the OGCMs. In addition, the diapycnal diffusivity is a central parameter for the behavior of the thermohaline circulation, but due to the strong numerical diffusion intrinsic to box models, the effects of diapycnal diffusivity cannot be examined.

The importance of the diapycnal diffusivity to the thermohaline circulation under relaxation boundary conditions has been discussed and widely examined in numerical models. Welander (1971) derived a scaling relationship for the zonal flow based on a vertical advective-diffusive balance and the thermal wind relation. F. Bryan (1987) postulated this to hold for the zonally averaged meridional flow as well, i.e, the meridional overturning should have a two-thirds power law dependence on the diapycnal diffusivity, even though he only got an approximate one-third power law in his numerical experiments. Marotzke (1997) limited diapycnal diffusion to act only in lateral boundaries and convection regions and found that when the vertical diffusivity $K$ is below $30 \mathrm{~cm}^{2} \mathrm{~s}^{-1}$, the meridional overturning strength is proportional to $K^{2 / 3}$. 
He went on to offer a theory relating the north-south density difference to the eastwest density difference; the latter is directly related to the shear of the meridional flow and thus the thermohaline circulation.

The thermohaline circulation under mixed boundary conditions has also been investigated. Weaver et. al. (1993) examined the effect of freshwater fluxes on the stability and variability of the thermohaline circulation. They found that the freshwater forcing is the dominant factor in determining the model's stability and internal variability. Increasing the relative importance of the freshwater flux versus the thermal forcing caused in turn, one stable steady state of the model, two stable states, one stable and one unstable equilibrium, or no stable steady states. In addition, Huang and Chou (1994) studied the parameter sensitivity of the saline circulation forced by freshwater flux alone. When the freshwater flux is increased from $0.01 \mathrm{~m}_{\text {year }}{ }^{-1}$ to $1 \mathrm{~m}_{\text {year }}{ }^{-1}$ with other parameters fixed, the system evolves from a steady state with no oscillations to a state of periodic oscillation whose frequency increases almost linearly with the amplitude of the freshwater flux. On the other hand, when the freshwater flux is fixed and the vertical mixing coefficient is increased from $0.5 \mathrm{~cm}^{2} \mathrm{~s}^{-1}$ to $2.5 \mathrm{~cm}^{2} \mathrm{~s}^{-1}$, the system evolves from a steady circulation to a state of single-period oscillation, a chaotic state, a single period state and finally to another chaotic state when the vertical mixing is larger than $2.0 \mathrm{~cm}^{2} \mathrm{~s}^{-1}$.

The thermohaline circulation in a double hemisphere model is investigated in Klinger and Marotzke (1998) and Marotzke and Klinger (1998) under different boundary conditions. They found that freshwater forcing is crucial for the stability and variability of the thermohaline circulation. Recently, Weber (1998) examined parameter sensitivity of a coupled atmosphere-ocean model under several types of upper boundary conditions. It was found that the magnitude of MOC basically scales with the half power law of the vertical diffusivity.

The importance of diapycnal mixing, and the relative lack of theoretical study of its role, motivated the scaling analysis under mixed boundary conditions in a single hemisphere model (Chapter 2). This analysis allows us to estimate the relative influence of diapycnal mixing, thermal forcing, freshwater forcing, the basin dimension and also the Coriolis parameter on the equilibrium of the thermohaline circulation. 


\subsubsection{Double Diffusion and Thermohaline Circulation}

In the following, it is shown that the large scale thermohaline circulation (thousands of kilometers in space and thousands of years in time) and small scale double-diffusive processes (centimeters to meters in space and minutes in time) are intimately related. The double-diffusive processes play a significant role in diapycnal mixing and water mass transformation, which will induce a response in the thermohaline circulation.

Gargett and Holloway (1992) investigated the sensitivity of the GFDL OGCM to a simple parameterization of double diffusion. They showed that the steady-state characteristics of low resolution GCMs used in climate studies are very sensitive to the ratio of the vertical eddy diffusivities for salinity and temperature $d=K_{S} / K_{T}$. With $0.5<d<2.0$, they found: (1) Major features of the steady-state model solutions vary greatly with $d$, including the magnitude and direction of the thermohaline circulation, as well as the intermediate and deep-water T/S properties and stability; (2) The effects on the model solutions are largely determined by the diffusivity ratio $d$, rather than the particular choice of $K_{S}$ and $K_{T}$ values; (3) The effects when $d \neq 1$ are nonlinear. Ruddick and Zhang (1989) found that the "salt oscillator" mechanism (Welander 1986) in a box model could be completely stabilized by incorporating salt fingers into the model. Recently, Gargett and Ferron (1996) found that multibox models with non-equal heat and salt diffusivities exhibited extended ranges of multiple equilibria, a different mode transition near present-day values of freshwater forcing magnitude, and the possibility of quasi-periodic oscillatory states compared to an equal diffusivity run. Similar parameterization for double-diffusive processes has also been used in the study of the formation and spread of the Mideterranean water (Spall, 1998; Gerdes et al. 1998). Climate models generally neglect the doublediffusive processes, so there is a need for expanded studies of their effects.

Since the Gargett and Holloway (1992) parameterizations of double diffusion are rather simple, one must be concerned that their studies might overestimate the impact of differential mixing. There is good evidence that the double-diffusive processes are most active only under certain hydrographic conditions. Although theoretically the vertical gradient of the unstable component is required to be about $1 \%$ of that of the stable component for both types of double diffusion to occur, only in places where $R_{\rho}$ is close to one, are salt fingers or diffusive layers actually observed. This 
suggests we need a more accurate parameterization of the occurrence and strength of double diffusion in numerical models. In this study, we adopt a parameterization for both types of double diffusion, in which the magnitudes of the vertical diffusivity of temperature and salinity are different and both strongly dependent on the local density ratio.

\subsection{Overview}

In this thesis, the thermohaline circulation and the effects of the double-diffusive processes are investigated theoretically and numerically.

In Chapter 2, a scaling analysis of the thermohaline circulation with uniform vertical mixing has been done under three types of upper boundary conditions, and tested using the Geophysical Fluid Dynamics Laboratory Modular Ocean Model 2(GFDL MOM2). Starting from the continuity equation, thermal-wind relation and the vertical advection-diffusion density balance, the strength of the MOC is found to depend upon the north-south surface density difference. Under the mixed boundary conditions, the north-south salinity difference (which accounts for part of the density difference), is directly related to the strength of MOC, through the salt conservation equation. As a result, multiple solutions are possible under mixed boundary conditions, and an algebraic relation is obtained to determine the existence of multiple equilibria, which depends on two uncertain parameters in the oceans: diapycnal diffusivity and freshwater forcing. Also, the stability of the solution is analyzed and comparison with the previous box model results are given.

Chapter 3 summarizes the parameterization for the double-diffusive processes (Schmitt 1981; Kelley, 1984, 1990), and the scaling analysis is used to investigate their impact on the thermohaline circulation under three types of boundary conditions. With the effective reduction in diapycnal density diffusivity by double-diffusive processes, a change in the thermohaline circulation is expected. With an assumption that the basin (vertical) density ratio is given by the surface horizontal density ratio, we can analyze the effects of the parameterization of double diffusion on the thermohaline circulation through scaling relations. Given the uncertainty of the variables associated with the double-diffusive parameterization, the sensitivity can be obtained analyti- 
cally, and a numerical evaluation is done by using GFDL MOM2. Chapter 3 gives the theoretical basis for the numerical experiments presented in Chapter 4 and Chapter 5 .

In Chapter 4, double diffusive mixing is implemented in GFDL MOM2 and its effect on the thermohaline circulation under relaxation boundary conditions is analyzed. Besides the quantitative difference on the MOC; the poleward heat transport, the water masses and the water column stability are also investigated. In particular, the underlying mechanisms for the changes are discussed.

Chapter 5 investigates the effects of double-diffusion on the thermohaline circulation under the mixed boundary conditions. Quasi-equilibrium experiments are used to investigate the critical freshwater forcing between runs with and without doublediffusion. The sensitivity to the variables in the parameterization of salt fingers is also investigated. Individual experiments with different fixed freshwater forcing are implemented, to investigate the impacts of double-diffusion over a range of parameter space.

Summaries and conclusions are presented in Chapter 6. 


\section{Chapter 2}

\section{Scaling Analysis and Numerical Experiments on the Thermohaline}

\section{Circulation}

\section{$2.1 \quad$ Introduction}

Box models and numerical models are the main tools available for study of the thermohaline circulation. In his simple and elegant box model, Stommel (1961) first recognized the possibility of multiple solutions of the thermohaline circulation. In a two-box one-hemisphere model, one box for the polar region and another for the equatorial region, he found that under certain conditions, there were three states possible: (1) a stable state which is thermally controlled (thermal mode), with a relative fast circulation, (2) a stable state which is salinity controlled (haline mode), with a relatively slow circulation and (3) an unstable state that is thermally controlled. F. Bryan (1986) proved the existence of multiple equilibria of the thermohaline circulation in a numerical general circulation model (GCM).

For the thermohaline circulation, the upper boundary conditions for temperature and salinity have been widely discussed. Since there is a strong feedback between the 
atmospheric temperature and sea surface temperature (SST), a relaxation boundary condition for the SST has been widely used (Haney, 1971):

$$
K \frac{\partial T}{\partial z}=\Gamma\left(T^{*}-T_{s}\right)
$$

where $K \partial T / \partial z$ is the surface heat flux carried by vertical diffusivity $K, T^{*}$ is the so called "apparent atmospheric temperature", which is determined by the sum of solar insolation, latent heat flux, sensible heat flux and turbulent heat flux, $T_{s}$ is SST, and $\Gamma$ is the inverse of the relaxation time, typically taken to be about ( 30 days $)^{-1}$ for a surface layer thickness of $50 \mathrm{~m}$. The strong feedback mechanism allows the SST to be maintained around the prescribed $T^{*}$.

Another option is the heat flux boundary condition on temperature:

$$
K \frac{\partial T}{\partial z}=\frac{Q}{\rho_{0} c_{p}} .
$$

Here $Q$ is the heat flux from atmosphere to ocean, $\rho_{0}$ and $c_{p}$ are density and the specific heat of sea water respectively.

For salinity, there are several choices. First we can relax the sea surface salinity to a prescribed distribution, analogous to temperature:

$$
K \frac{\partial S}{\partial z}=\Gamma_{S}\left(S^{*}-S_{s}\right) .
$$

This condition is very easy to use but it is hardly justified physically, because there is no such feedback between $S^{*}$ and $S_{s}$. The second way is to use a virtual salt flux condition for salinity, i.e, from evaporation minus precipitation and runoff $(e-p)$, we obtain an equivalent salt flux on the surface. The mean surface salt balance is thus (Stern, 1975):

$$
K \frac{\partial S}{\partial z}=(e-p) \bar{S}
$$

where $e-p$ is evaporation minus precipitation, including runoff, and $\bar{S}$ is the averaged salinity at the surface. This condition, an approximation to the freshwater flux condition, is being used in an increasing number of ocean models. Huang (1993) proposed a more realistic upper boundary condition, the so-called "natural" boundary condition on salinity. Due to the restraint of the free surface requirement, this kind of boundary condition is not discussed here, but the scaling argument should be similar to the virtual salt flux case. For simplicity, wind stress effects are neglected in the 
scaling argument and in most of the numerical experiments. We call the combination of (2.1) and (2.3) "relaxation" boundary conditions; (2.2) and (2.4) are called "flux" boundary conditions; and in comparison, (2.1) and (2.4) are called "mixed" boundary conditions.

Previous studies suggest that the freshwater flux and the diapycnal diffusivity are very important parameters to the thermohaline circulation, yet both are poorly known from observations. In the following, scaling analysis and numerical experiments are used to understand the sensitivity of the thermohaline circulation to these parameters. In Section 2.2, the scaling analyses of the thermohaline circulation under three different kinds of upper boundary conditions are provided. The numerical model used in the study is introduced in Section 2.3. In Section 2.4 numerical experiments are implemented to compare with the scaling results. Section 2.5 contains a summary and discussion.

\subsection{Scaling Analysis}

\subsubsection{Equations}

Similar to Welander (1971), we start from the following equations:

(i) the incompressible condition:

$$
\frac{\partial u}{\partial x}+\frac{\partial v}{\partial y}+\frac{\partial w}{\partial z}=0
$$

(ii) the thermal wind relation:

$$
\frac{\partial \vec{u}}{\partial z}=-\frac{g}{\rho_{0} f} \vec{k} \times \nabla \rho
$$

(iii) the vertical advective-diffusive balance of density:

$$
w \frac{\partial \rho}{\partial z}=K \frac{\partial^{2} \rho}{\partial z^{2}}
$$

where $\vec{u}=u \vec{i}+v \vec{j}$ and $w$ are the horizontal and vertical components of velocity respectively, $f$ is the Coriolis parameter, $\rho$ is the density, $K$ is the assumed uniform diapycnal diffusivity of density, and $g$ is the gravitational acceleration. 
Assumption (i) is used widely in oceanic theoretical and numerical studies, and (ii) comes from the geostrophic and hydrostatic relations, which are true for the largescale thermohaline circulation. Assumption (iii) has been invoked by Munk (1966) and justified by the correspondence of observed vertical profiles of salinity and temperature to the model (between 1 and $5 \mathrm{~km}$ depth); in addition, Munk and Wunsch (1998) have revisited this issue. This assumption is implied in the Stommel and Arons (1960) deep ocean circulation theory and was used in the scaling arguments of Welander (1971) and F. Bryan (1987).

From the above equations, we obtain the scaling relations:

$$
\begin{aligned}
U D & =W L \\
\frac{\triangle \rho}{\rho_{0}} & =\frac{f U L}{g D}, \\
D & =\frac{K}{W}
\end{aligned}
$$

where $U(L)$ and $W(D)$ are horizontal and vertical velocity (length) scales respectively, and $\triangle \rho$ is the north-south density difference at the ocean surface.

We can then define a vertical scale in terms of $\Delta \rho / \rho$ :

$$
D=\left[\frac{f K L^{2}}{g \triangle \rho / \rho_{0}}\right]^{1 / 3} .
$$

The strength of the meridional overturning $M=U D L$ is given by

$$
\begin{aligned}
M & =W L^{2} \\
& =\left[\frac{g \triangle \rho K^{2} L^{4}}{\rho_{0} f}\right]^{1 / 3} .
\end{aligned}
$$

Note that $M$ is determined by the meridional velocity $v$, which is related to the east-west density difference, rather than the north-south density difference. Since in the thermocline, the velocity components $u$ and $v$ are of the same order, and $u$ is determined by the north-south density difference, we infer that $v$ has the same order of magnitude. Marotzke (1997) provides a discussion in which the east-west density difference is closely related to the north-south surface density difference. However, he assumed vertical mixing only occurs in the lateral boundary regions which is different from the uniform mixing assumed here. To the lowest order, we can assume (2.12) is an acceptable estimate for the strength of the meridional overturning cell. 
The poleward heat transport is very important for climate studies; this can be scaled as follows:

$$
Q=C_{0} \rho_{0} c_{p} M \Delta T \propto K^{2 / 3},
$$

where $c_{p}$ is the specific heat under constant pressure, and the north-south temperature difference $\Delta T$ is used to represent the temperature difference between the deep and surface flows. We use both maximum $M$ and $\Delta T$ to estimate $Q$, however, in the real ocean (a single-hemisphere basin), maximum $M$ occurs with minimum $\Delta T$, and maximum $\Delta T$ lies in the minimum $M$ region. As a result, the maximum poleward heat transport is found in mid-latitudes. Thus, a constant factor $C_{0}$ is introduced here to represent this effect. For the simplest case, we can assume that $Q$ reaches its maximum at mid-latitudes where both $\Delta T$ and $M$ are assumed half of their maximum values respectively. This suggests an approximate value of $C_{0}=1 / 4$.

Furthermore, assuming a linear equation of state

$$
\rho=\rho_{0}(1-\alpha T+\beta S),
$$

the ratio $\triangle \rho / \rho_{0}$ in (2.11) and (2.12) can be replaced by

$$
\triangle \rho / \rho_{0}=|\alpha \triangle T-\beta \triangle S|,
$$

where $\triangle T$ and $\triangle S$ are north-south temperature and salinity difference respectively.

\subsubsection{Relaxation Boundary Conditions}

In most cases in the literature that used relaxation boundary conditions, both $\Gamma$ and $\Gamma_{s}$ were quite large, so that $\Delta T$ and $\Delta S$ can be approximated by $\Delta T^{*}$ and $\Delta S^{*}$ respectively, where $\Delta T^{*}$ and $\Delta S^{*}$ are the north-south temperature and salinity differences in the prescribed values $T^{*}$ and $S^{*}$. This assumption will be used in the following analysis.

We find it useful to define the horizontal density ratio:

$$
R=\frac{\alpha \triangle T}{\beta \triangle S} .
$$

In the present-day oceans, the water is cold and fresh in the polar regions, and warm and salty in the equatorial regions, thus temperature and salinity have opposite effects 
on the surface density difference. When $R>1$, the temperature dominates the density difference, and the densest water is formed and sinks in the polar regions. The thermohaline circulation is in the "thermal" mode. In contrast, when $R<1$, the salinity dominates the density difference, and the densest water is formed and sinks in the equatorial regions. This thermohaline circulation is in the "haline" mode.

Using (2.16), the depth scale, the meridional overturning rate, and the poleward heat transport become

$$
\begin{aligned}
D & =\left[\frac{f K L^{2} R}{g \alpha \Delta T|R-1|}\right]^{1 / 3}, \\
M & =\left[\frac{g \alpha \Delta T|R-1| K^{2} L^{4}}{f R}\right]^{1 / 3}, \\
Q & =C_{0} \rho_{0} c_{p} \Delta T M
\end{aligned}
$$

respectively.

With the relaxation boundary conditions, $\Delta T$ and $\Delta S$ are very close to the constant $\Delta T^{*}$ and $\Delta S^{*}$. As a result, the north-south density difference is nearly fixed, so there is only one thermohaline circulation mode. The above power laws have been discussed previously (Welander, 1971; F. Bryan, 1987), and are reproduced here as a first step for the case under mixed boundary conditions. In addition, the above conclusions will be examined in a numerical model in Section 2.4.

\subsubsection{Flux Boundary Conditions}

\section{North-south Salinity Difference}

Here we assume flux boundary conditions (2.2), (2.4). Unlike the relaxation boundary conditions, the north-south salinity (temperature) difference is no longer fixed, but rather, it depend on the strength of the meridional overturning rate, and thus becomes part of the solution we are pursuing.

Consider a two-box model of the ocean (Stommel, 1961), in which a polar box and an equatorial box are well mixed and connected by pipes at the top and the bottom. Defining $\bar{S}_{e}$ and $\bar{S}_{p}$ as salinities in the equatorial and polar boxes respectively, the 
salt conservation equations are

$$
\begin{aligned}
& \dot{\bar{S}}_{e}=\frac{\bar{E}_{e} \bar{S}}{H}-\frac{M_{0}}{H L^{2} / 2}\left(\bar{S}_{e}-\bar{S}_{p}\right) \\
& \dot{\bar{S}}_{p}=\frac{\bar{E}_{p} \bar{S}}{H}+\frac{M_{0}}{H L^{2} / 2}\left(\bar{S}_{e}-\bar{S}_{p}\right)
\end{aligned}
$$

where $\bar{E}_{e}, \bar{E}_{p}$ are the evaporation minus precipitation rates in the equatorial and polar box respectively, $L^{2}$ is the horizontal area of the whole ocean basin, and $M_{0}$ is the volume flux between the two boxes, $H$ is the depth of the ocean, $\bar{S}$ is the averaged salinity over the whole basin, and "." denotes the time derivative.

From the above two equations, we can derive an equation governing evolution of the salinity difference, $\Delta S_{1}=\bar{S}_{e}-\bar{S}_{p}$,

$$
\Delta \dot{S}_{1}=\frac{4}{H}\left(\frac{\bar{E}_{e}-\bar{E}_{p}}{4} \bar{S}-\frac{M_{0}}{L^{2}} \Delta S_{1}\right) .
$$

To obtain a more accurate estimate of the north-south salinity difference in the scaling analysis, we make the following assumptions:

$$
\begin{aligned}
\Delta S & =2 \Delta S_{1} \\
E_{e}-E_{p} & =2\left(\bar{E}_{e}-\bar{E}_{p}\right) \\
M & =2 M_{0}
\end{aligned}
$$

where $\Delta S, E_{e}-E_{p}$ and $M$ are used to represent the corresponding quantities in more complicated models, like numerical models, rather than that from a two-box model which is strongly averaged. The above extension from a box model to a more realistic model is based on simple linear assumptions, which may differ from the real situation, but as a scaling argument, we believe it reflects the lowest order approximation. Thus $\Delta S\left(E_{e}-E_{p}\right)$ is the salinity (freshwater) difference between northern and southern regions, and $M$ is the maximum of the meridional overturning. For convenience, we introduce

$$
E=\left(E_{e}-E_{p}\right) / 2
$$

to represent the magnitude of freshwater forcing. Then we have

$$
\Delta \dot{S}=\frac{2}{H}\left(E \bar{S}-\frac{M}{L^{2}} \Delta S\right)
$$


For a steady state, we obtain

$$
\Delta S_{0}=\frac{E L^{2}}{M} \bar{S}=\frac{\bar{S} E}{W}=\frac{\bar{S} E D}{K} .
$$

A similar scaling result has been obtained in Huang and Chou (1994). We can see that $\Delta S_{0}$ is proportional to the magnitude of the freshwater forcing and inversely proportional to the strength of the meridional overturning circulation rate.

Similarly, we obtain an expression for the north-south temperature difference

$$
\Delta \dot{T}=\frac{2}{H}\left(\frac{Q}{\rho_{0} c_{p}}-\frac{M}{L^{2}} \Delta T\right)
$$

For a steady state, we have

$$
\Delta T_{0}=\frac{Q L^{2}}{M \rho_{0} c_{p}}
$$

\section{Scaling Analysis and Solutions}

With (2.28) and (2.30), the north-south density difference becomes

$$
\begin{aligned}
\Delta \rho / \rho_{0} & =\alpha \Delta T_{0}-\beta \Delta S_{0} \\
& =\frac{L^{2}}{M}\left|\frac{\alpha Q}{\rho_{0} c_{p}}-\beta \bar{S} E\right| \\
& =\frac{\beta \bar{S} E L^{2}}{M}|R-1|
\end{aligned}
$$

where

$$
R=\frac{\alpha Q}{\rho_{0} c_{p} \beta \bar{S} E}
$$

is the density ratio.

Substituting (2.31) into (2.11) and (2.12), we have

$$
\begin{aligned}
D & =\left[\frac{f L^{2} K^{2}}{g \beta \bar{S} E|R-1|}\right]^{1 / 4}, \\
M & =\left[\frac{g L^{6} K^{2} \beta \bar{S} E|R-1|}{f}\right]^{1 / 4} .
\end{aligned}
$$

When $R=0$, i.e., there is freshwater forcing only, the above expressions reduce to those of Huang and Chou (1994). 
Therefore under flux boundary conditions, the magnitude of the meridional overturning is proportional to the half power law of the diapycnal diffusivity. Similar to the relaxation boundary conditions, when $R>1$, the thermal forcing dominates and the thermohaline circulation lies in a "thermal" mode. In contrast, when $R<1$, we have a "haline" circulation mode.

\section{Stability Analysis}

From equation (2.27) and (2.29), we can derive the equation for the north-south density difference, by utilizing a linear density equation:

$$
\begin{aligned}
\Delta \dot{\rho} & =-\alpha \Delta \dot{T}+\beta \Delta \dot{S} \\
& =\frac{2}{H}\left[\left(\beta E \bar{S}-\frac{\alpha Q}{\rho_{0} c_{p}}\right)-\frac{M}{L^{2}} \Delta \rho\right]
\end{aligned}
$$

We then separate the meridional overturning and north-south density difference into two parts respectively:

$$
\begin{aligned}
M & =M_{0}+M^{\prime}, \\
\Delta \rho & =\Delta \rho_{0}+\Delta \rho^{\prime},
\end{aligned}
$$

where $M_{0}$ and $\Delta \rho_{0}$ are the steady solutions discussed above, and $M^{\prime}$ and $\Delta \rho^{\prime}$ are perturbations.

From equation (2.12), we have

$$
\frac{M^{\prime}}{M_{0}}=\frac{\Delta \rho^{\prime}}{3 \Delta \rho_{0}}
$$

Substituting (2.35) - (2.37) into (2.34), we obtain

$$
\Delta \dot{\rho^{\prime}}=-\frac{8 M_{0}}{3 H L^{2}} \Delta \rho^{\prime}
$$

Therefore, the thermohaline circulation under flux boundary conditions is predicted to be stable. 


\subsubsection{Mixed Boundary Conditions}

Here we assume mixed boundary conditions $(2.1),(2.4)$. The north-south temperature difference is basically fixed by the prescribed values, as under relaxation boundary conditions. But the north-south salinity difference is determined by (2.27), the same as the flux boundary conditions.

\section{Scaling Analysis and Solutions}

With $\Delta S_{0}$ given in (2.28), we obtain:

$$
\frac{\triangle \rho}{\rho_{0}}=\left|\alpha \triangle T-\frac{\beta \bar{S} E D}{K}\right| .
$$

From this we can conclude that when

$$
\alpha \triangle T>\frac{\beta \bar{S} E D}{K},
$$

the density difference is thermally-dominant. Alternatively, when the thermal effect is less than the saline effect, the density difference is salt-dominant.

Substituting (2.39) into (2.11), we obtain a quartic equation for $D$

$$
\frac{f L^{2} K}{g D^{3}}=\left|\alpha \Delta T-\frac{\beta \bar{S} E D}{K}\right| .
$$

Since the salinity difference is now related to the vertical length scale, the relation between the depth scale and the external parameters becomes more complicated.

Introducing the following nondimensional variables:

$$
\begin{aligned}
F & =\frac{g K^{4}(\alpha \Delta T)^{4}}{f L^{2} E^{3}(\beta \bar{S})^{3}}, \\
R & =\frac{\alpha \Delta T K}{\beta \bar{S} E D},
\end{aligned}
$$

equation (2.41) becomes

$$
R^{4}=F|R-1| .
$$

Using (2.28), $R$ can be written as:

$$
R=\frac{\alpha \Delta T}{\beta \Delta S_{0}},
$$


which is the horizontal density ratio representing the relative contributions of salt and heat to the surface density. When $R>1$, the thermal forcing dominates and we have thermal modes; on the other hand, when $R<1$, the freshwater forcing dominates and we obtain a haline mode.

$F$ is a very complicated nondimensional number, and we can write it in the form of

$$
F=\left[R \frac{D}{D_{T}}\right]^{3}
$$

where $R$ is the horizontal density ratio, $D_{T}$ is the depth scale with only thermal forcing, i.e,

$$
D_{T}=\left[\frac{f L^{2} K}{g \alpha \Delta T}\right]^{1 / 3}
$$

and $D$ is the depth scale with both thermal and freshwater forcing. As shown in equation (2.44), $F$ is the only external parameter to determine $R$, thus it is essential for the behavior of the thermohaline circulation. Nondimensional number $F$ not only includes the comparison of freshwater forcing versus thermal forcing, but also the diapycnal diffusivity and basin dimensions in a complicated form.

First we discuss two limits:

(a) $R \gg 1$

In this case, freshwater forcing is negligible compared with the thermal forcing, and the problem reduces to a case where only thermal forcing under the relaxation boundary condition is applied in the model. Then equation (2.44) can be reduced to

$$
R^{3}=F
$$

Thus we have

$$
\begin{aligned}
R & =F^{1 / 3} \\
D & =\left[\frac{f L^{2} K}{g \alpha \Delta T}\right]^{1 / 3} \\
M & =\left[\frac{g \alpha \Delta T L^{4} K^{2}}{f}\right]^{1 / 3}
\end{aligned}
$$

This result is the same as we obtained under the relaxation boundary conditions with $\Delta S=0$. 
(b) $R \ll 1$

In this limit, thermal forcing is negligible compared with the freshwater forcing, then equation (2.44) is reduced to

$$
R^{4}=F
$$

Thus we obtain

$$
\begin{aligned}
R & =F^{1 / 4} \\
D & =\left[\frac{f L^{2} K^{2}}{g \beta \bar{S} E}\right]^{1 / 4} \\
M & =\left[\frac{g \beta \bar{S} E L^{6} K^{2}}{f}\right]^{1 / 4}
\end{aligned}
$$

This is identical with the results we got under flux boundary conditions with $Q=0$. Next, we solve the full equation (2.44) in the following two cases:

(i) Thermal mode(s) equations

$$
\left\{\begin{array}{l}
R^{4}-F R+F=0 \\
R>1
\end{array}\right.
$$

(ii) Haline mode(s) equations

$$
\left\{\begin{array}{l}
R^{4}+F R-F=0 \\
R<1
\end{array}\right.
$$

The detailed calculation and discussion can be found in the appendix; here we summarize the results.

The nature of the solution to equation (2.44) depends on the size of $F$ relative to a critical parameter:

$$
F_{c}=\frac{4^{4}}{3^{3}}=\frac{256}{27} .
$$

When $F>F_{c}$, two thermal modes and one haline mode are possible; when $F<F_{c}$, only one haline mode can exist.

The above solutions are based on $R$, and then we can calculate the meridional overturning by (2.12) and the poleward heat transport by (2.13). 
Assuming the thermal forcing is fixed $(\Delta T=$ const), (2.58) implies a critical value for the freshwater forcing:

$$
\begin{aligned}
E_{c}(K) & =\left[\frac{27 g(\alpha \Delta T)^{4} K^{2}}{256 f L^{2}(\beta \bar{S})^{3}}\right]^{1 / 3} \\
& =C K^{2 / 3}
\end{aligned}
$$

For a given value of $K$, there is an upper limit of freshwater forcing $E_{c}(K)$, beyond which no thermal mode can exist. Below this value two thermal modes, in addition to the haline mode, are possible for all given conditions.

The existence of an upper limit on the thermal mode can be understood as follows. The freshwater forcing acts as a brake on the thermally driven overturning circulation. For example, with $E>0$, we know the maximum meridional overturning is smaller than that without freshwater forcing, i.e.,

$$
M<\left[\frac{g \alpha \Delta T L^{4} K^{2}}{f}\right]^{1 / 3}=M_{T H},
$$

where $M_{T H}$ is the meridional overturning due to thermal forcing alone. Using (2.28),

$$
\begin{aligned}
\Delta S_{0} & =\frac{L^{2} \bar{S}}{M} E, \\
& >\frac{L^{2} \bar{S}}{M_{T H}} E .
\end{aligned}
$$

For a given thermal forcing $\Delta T, \Delta S_{0}$ increases with larger $E$; thus when $E$ is large enough, $\Delta S_{0}$ can overcome the density effect of $\Delta T$, eliminating the thermal mode.

We plot the critical line (2.60) in Figure 2.1 (a), where the parameters are taken as

$$
\begin{aligned}
\alpha & =2.0 \times 10^{-4} \mathrm{~K}^{-1} \\
\beta & =8.0 \times 10^{-4} \mathrm{psu}^{-1} \\
\Delta T & =20 \mathrm{~K} \\
\bar{S} & =35 \mathrm{psu} \\
f & =1.0 \times 10^{-4} \mathrm{~s}^{-1} \\
L & =6.0 \times 10^{6} \mathrm{~m} \\
g & =9.8 \mathrm{~m}^{2} \mathrm{~s}^{-1} .
\end{aligned}
$$


In the region below the critical curve, three modes are possible: two thermal modes and one haline mode, while in the region above it, only one haline mode can exist.

The meridional overturning rates corresponding to the thermal modes and haline mode are plotted in Figure 2.1 (b)-(d) respectively. For the thermal mode in Figure 2.1 (c) (as shown next, this is an unstable solution), the overturning rate depends strongly on the freshwater forcing while being nearly independent of the diapycnal diffusivity. For the other thermal mode in Figure 2.1 (d) (which will be proved to be a stable solution), the magnitude of the overturning is larger than that in the unstable thermal mode, and it is more sensitive to the magnitude of diapycnal diffusivity than the freshwater flux. The existence of the above two thermal modes depends on the relative relation of $K$ and $E$, as given in the critical relation (2.60). For the haline mode, it shows different features in different regions. In the multiple equilibria region, the magnitude of the thermohaline circulation is mainly a function of freshwater forcing, while in the single equilibrium region, it changes with both diapycnal diffusivity and the freshwater forcing. In the low diffusivity region, it changes mainly with diapycnal diffusivity. Note in the multiple equilibria region, the stable thermal mode is strongest.

Moreover, we can plot the dependence of the meridional overturning on $E$ and $K$ respectively (Figure 2.2). For a given $K=1 \mathrm{~cm}^{2} \mathrm{~s}^{-1}$, there is a bifurcation point at about $E=1 \mathrm{~m}$ year ${ }^{-1}$, beyond which only one haline mode is possible.

The above arguments are based on the assumption of a specified equator-pole temperature difference. However, since the atmosphere and oceans constitute a coupled system, the temperature difference $\Delta T$ could change. From (2.59), a change in $\Delta T$ will lead to a different critical relation between diapycnal diffusivity and hydrologic forcing. Past climatic states of the Earth may have had significantly different $\Delta T$ and $E$, and both must be considered in evaluating the possible modes of the thermohaline circulation. 
(a)

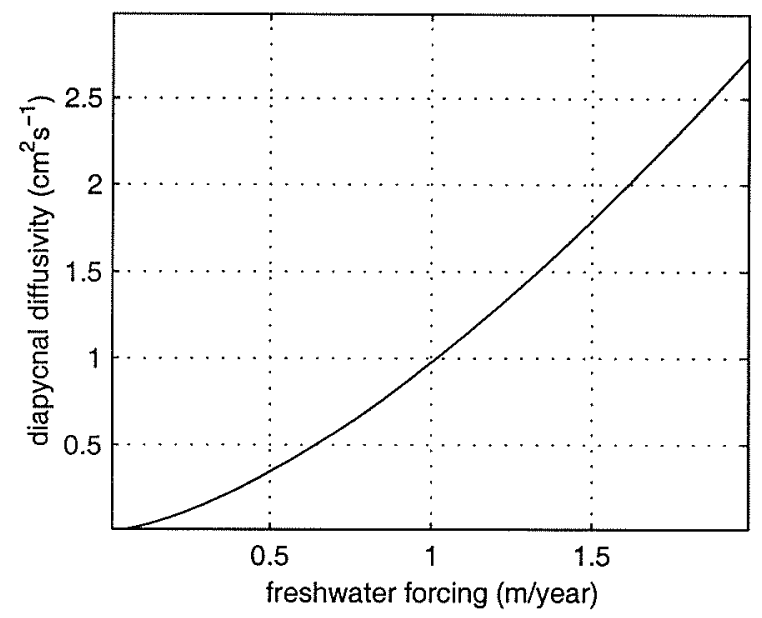

(c) Unstable Thermal Mode

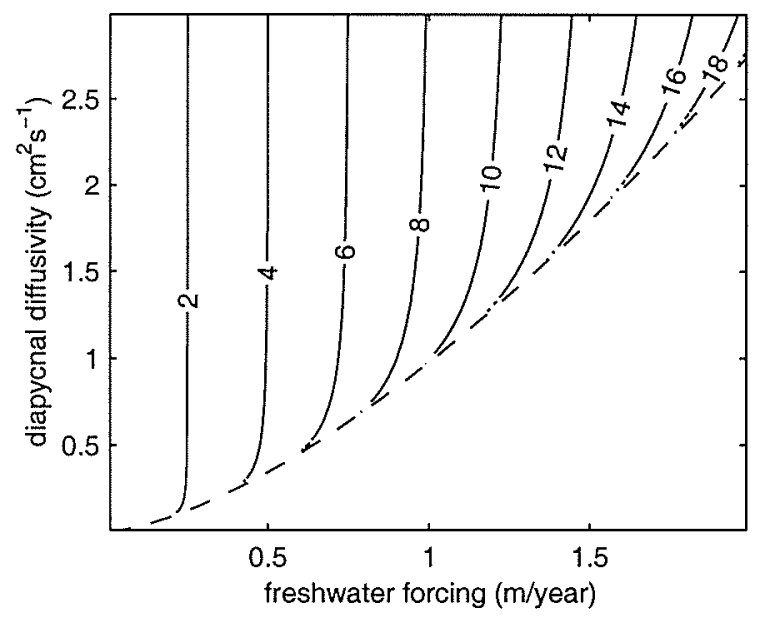

(b) Haline Mode

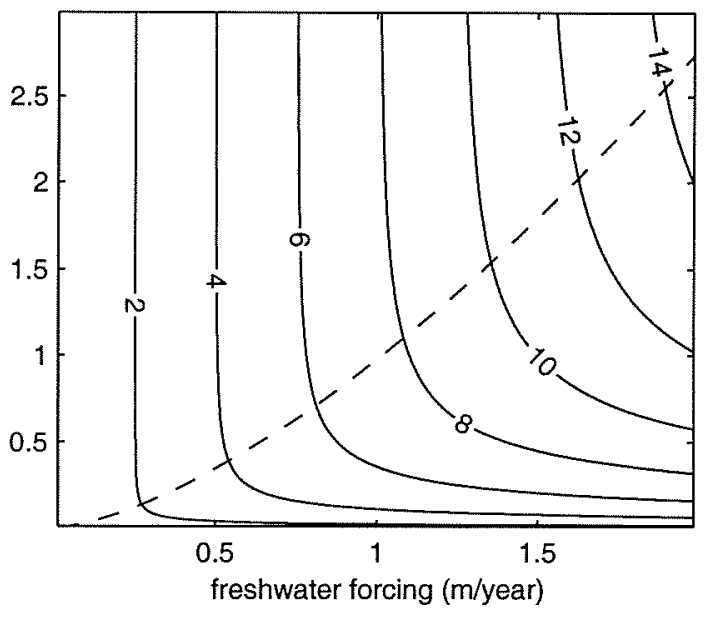

(d) Stable Thermal Mode

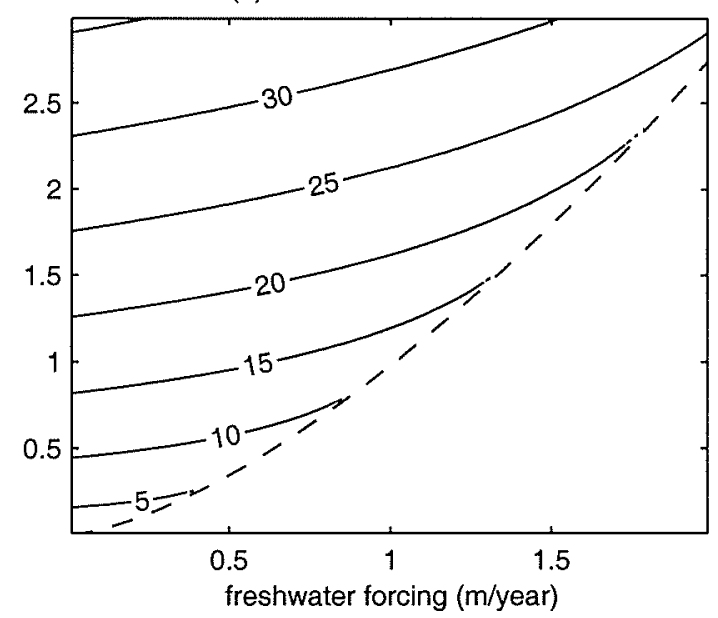

Figure 2.1: Criterion and multiple solutions of the thermohaline circulation under mixed boundary conditions. (a) The critical curve $F=256 / 27$ and the meridional overturning rates of (b) the haline mode, (c) the unstable thermal mode and (d) the stable thermal mode. 

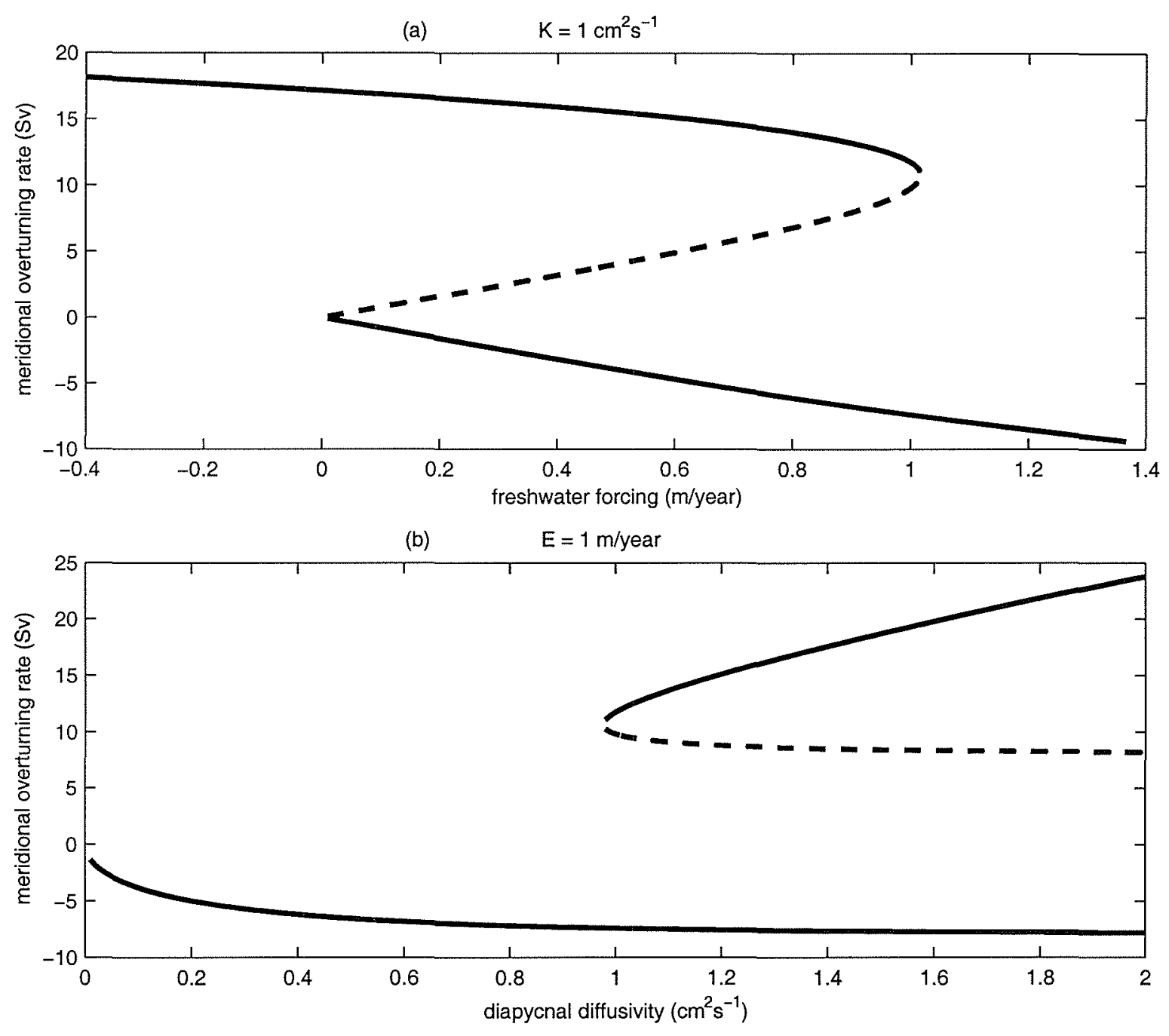

Figure 2.2: Solutions of meridional overturning rate under mixed boundary conditions for (a) fixed $K=1.0 \mathrm{~cm}^{2} \mathrm{~s}^{-1}$ with changing $E$ and (b) fixed $E=1 \mathrm{~m}$ year ${ }^{-1}$ with changing $K$. The upper solid line corresponds to the stable thermal mode, while the lower solid line is the solution for the haline mode, and the dotted line represents the result for the unstable thermal mode. For $K=1 \mathrm{~cm}^{2} \mathrm{~s}^{-1}$, there is a bifurcation point at about $E=1 \mathrm{~m}$ year $^{-1}$, beyond which only one haline mode is possible. The parameters are defined in (2.63). 


\section{Stability Analysis}

Now we can analyze the stability of the solutions we obtained. We write $\Delta S$ in two parts:

$$
\Delta S=\Delta S_{0}+\Delta S^{\prime}
$$

where $\Delta S_{0}$ is the steady solution and $\Delta S^{\prime}$ is the small perturbation.

Similarly, we assume the meridional overturning will change correspondingly:

$$
M=M_{0}+M^{\prime}
$$

Substituting equation (2.64) and (2.65) into (2.27), we obtain

$$
\begin{aligned}
\Delta \dot{S}^{\prime} & =\frac{2 E \bar{S}}{H}-\frac{2\left(M_{0}+M^{\prime}\right)}{H L^{2}}\left(\Delta S+\Delta S^{\prime}\right) \\
& =-\frac{2 M_{0}}{H L^{2}}\left(\Delta S^{\prime}+\frac{M^{\prime}}{M_{0}} \Delta S_{0}\right)
\end{aligned}
$$

where the high-order perturbation term $M^{\prime} \Delta S^{\prime}$ is neglected.

From equation (2.12), we derive

$$
\frac{M^{\prime}}{M_{0}}=-\frac{1}{3} \frac{\beta \Delta S^{\prime}}{\alpha \Delta-\beta \Delta S_{0}}
$$

Combining (2.66) and (2.67), we obtain

$$
\begin{aligned}
\Delta \dot{S}^{\prime} & =\frac{2(4-3 R)}{3(R-1)} \frac{M_{0}}{H L^{2}} \Delta S^{\prime} \\
& =A \Delta S^{\prime}
\end{aligned}
$$

where

$$
\begin{aligned}
R & =\frac{\alpha \Delta T}{\beta \Delta S_{0}} \\
A & =\frac{2(4-3 R)}{3(R-1)} \frac{M_{0}}{H L^{2}} .
\end{aligned}
$$

For the thermal mode with

$$
1<R<4 / 3
$$


we obtain

$$
A>0
$$

therefore this solution is unstable: a perturbation $\Delta S^{\prime} *$ is predicted to increase with time. In comparison, for the other thermal mode, $R>4 / 3$ and the haline mode, $R<1$, we have

$$
A<0
$$

thus this thermal mode and the haline mode are both stable solutions.

\section{Application to North Atlantic and North Pacific}

The above scaling argument is based upon a square basin. For a rectangular basin (i.e, $L_{x} \neq L_{y}$ ), we can replace the horizontal area $L^{2}$ by $L_{x} L_{y}$.

Given the uncertainty associated with both the diapycnal diffusivity and $e-p$ over the oceans, it is unrealistic to compare the current scaling argument with the real oceans in any detail. However, a rough estimate can be made based on presently available information. A map of evaporation minus precipitation over the global ocean is plotted in Figure 2 of Schmitt (1995) and is used for the estimate of $e-p$. Here we focus on the northern hemisphere where the North Atlantic and North Pacific are isolated and most appropriate for our scaling argument. Due to the effect of the ITCZ (Intertropical Convergence Zone), there is more precipitation than evaporation in the equatorial region, thus the maximum $e-p$ lies near $15^{\circ} \mathrm{N}$ for the North Atlantic and $20^{\circ} N$ for the North Pacific. The minimum $e-p$ (or maximum $p-e$ ) lies in the polar region of both oceans, close to $60^{\circ} \mathrm{N}$. As an approximation, we take $L_{y}=5 \times 10^{6} \mathrm{~m}$ for both oceans and $L_{x}=6 \times 10^{6} \mathrm{~m}$ for North Atlantic and $L_{x}=12 \times 10^{6} \mathrm{~m}$ for North Pacific. Then we estimate $\Delta T$ and $\bar{S}$ for both oceans from the Levitus (1982) climatology between the maximum and minimum $e-p$ regions respectively. We obtain approximate values of $\Delta T=18^{\circ} \mathrm{C}, \bar{S}=36 \mathrm{psu}$ for the North Atlantic and $\Delta T=20^{\circ} C, \bar{S}=34$ psu for the North Pacific (Table 2.1).

Compared to $e-p$, the diapycnal diffusivity is perhaps even less well known. Recent observations indicate strong spatial variability of diapycnal mixing in the oceans. Ledwell et al. (1993) found that the diapycnal diffusivity is about $0.15 \mathrm{~cm}^{2} \mathrm{~s}^{-1}$ in the upper thermocline in a tracer release experiment, while the diapycnal diffusivity is 
much larger close to rough topography in the abyss (approaches $100 \mathrm{~cm}^{2} \mathrm{~s}^{-1}$, Toole et al., 1994; Polzin et al., 1997). The basin-scale budget estimates require an averaged diapycnal diffusivity of about $1 \mathrm{~cm}^{2} \mathrm{~s}^{-1}$ (Munk, 1966; Munk and Wunsch, 1998). For simplicity, we adopt the canonical value of $K=1 \mathrm{~cm}^{2} \mathrm{~s}^{-1}$ for both basins in the scaling estimate here, however, the uncertainty of this value should be emphasized.

Using the above parameters, we estimate the critical evaporation minus precipitation rate as

$$
E_{c N A}=0.92 \text { year }^{-1}
$$

for the North Atlantic ocean and

$$
E_{c N P}=0.89 \text { year }^{-1}
$$

for the North Pacific Ocean. The critical values of freshwater forcing are surprisingly close for both oceans.

\begin{tabular}{|c|c|c|}
\hline & N. Atlantic & N. Pacific \\
\hline$L_{x}\left(10^{6} \mathrm{~m}\right)$ & 6 & 12 \\
\hline$\Delta T\left({ }^{\circ} \mathrm{C}\right)$ & 18 & 20 \\
\hline $\bar{S}(p s u)$ & 36 & 34 \\
\hline$K\left(\mathrm{~cm}^{2} \mathrm{~s}^{-1}\right)$ & 1 & 1 \\
\hline$\overline{E_{c}\left(m \text { year }^{-1}\right)}$ & 0.92 & 0.89 \\
\hline $\bar{E}\left(m\right.$ year $\left.^{-1}\right)$ & 0.75 & 1.05 \\
\hline scaling mode & multiple & single (haline) \\
\hline
\end{tabular}

Table 2.1: Comparison of the North Atlantic and North Pacific

From the Schmitt (1995) global ocean $e-p$ map, we find $E_{N A}$ is about $0.75 m y_{e a r}^{-1}$ for the North Atlantic Ocean and $E_{N P}$ is about 1.05 mear ${ }^{-1}$ for the North Pacific Ocean. The estimated freshwater forcing is much greater in North Pacific than in North Atlantic. Since $E_{N A}<E_{c N A}$, the scaling suggests the North Atlantic Ocean is in the multiple equilibria region. Currently the North Atlantic Deep Water (NADW) is formed in the polar region and exported equatorward, characteristic of a thermal mode. In contrast, for the North Pacific, where $E_{N P}>E_{c N P}$, no thermal mode is possible by the scaling argument. This is in line with the lack of deep water formation in the Pacific polar regions. The results are summarized in Table 2.1. 
Warren (1983) noted the effects of low sea surface temperature on the diminution of evaporation, and suggests that this causes the lack of bottom water formation in the Pacific compared to the North Atlantic. Here we find it is also possible that the larger gradient of $e-p$ in the North Pacific inhibits a thermal mode overturning cell. The stronger freshwater forcing and lower critical $E$ value for the North Pacific both inhibit the thermal mode. Compared to the North Atlantic Ocean, even though the higher north-south temperature gradient and lower mean salinity act to increase the critical $E$ value, the large size in the zonal dimension surpasses the above effects and leads to a smaller critical value in the North Pacific than the North Atlantic. Thus it is easier for the Pacific to destabilize the thermal mode even with the same freshwater forcing. In addition, there could well be significant differences in diapycnal mixing rates in the two basins due to varying bathymetry and tidal forcing (Polzin et al., 1997; Munk and Wunsch, 1998).

The individual oceans, however, are not isolated systems, moreover there is interaction between the oceans and the atmosphere. As a result, there is strong feedback between $\Delta T, E$ and the strength of the thermohaline circulation. Thus, parameters $\Delta T, E$ etc are not givens, but rather are part of the solution of the more complicated coupled system. Hopefully, the simple scaling argument given here provides the lowest order estimate of the behavior of the thermohaline circulation. Based upon the rough observations available, it is consistent with the current oceanic situation.

\section{Comparison with Box Model Results}

In the pioneering work of Stommel (1961), he found that under certain conditions, three states are possible, of which one thermal mode is stable, one thermal mode is unstable and the other haline mode is stable. Otherwise only one stable haline mode is obtained.

Marotzke (1990) used a two-box model similar to Stommel (1961), in which he defined the following nondimensional parameter and variable:

$$
\begin{aligned}
F_{M} & =\frac{\beta H_{S}}{K_{M}(\alpha \Delta T)^{2}} \\
R_{M} & =\frac{\alpha \Delta T}{\beta \Delta S}
\end{aligned}
$$


where $H_{S}$ is the equivalent salt flux, and $K_{M}$ is the linear proportionality coefficient between the inter-box flow and the density difference. He found that for $F_{M}<0.25$, three steady states exist, one haline mode and two thermal modes. Beyond $F_{M}=$ 0.25 , only one haline mode is a solution. In addition, for $R_{M}>2.0$ or $R_{M}<1.0$, the solution is stable, and for $1.0<R_{M}<2.0$, the solution is unstable. Therefore in the multiple equilibria region $\left(F_{M}<0.25\right)$, there is one stable thermal mode, one unstable thermal mode and one stable haline mode, and when $F_{M}>0.25$, there is only one stable haline mode.

Huang et al. (1992) studied the structure and stability of the multiple equilibria of the thermohaline circulation using $2 \times 2$ and $3 \times 2$ box models. They defined the following nondimensional number

$$
p=\frac{\Gamma(E-P)}{\rho_{0} c_{p}}
$$

where $\Gamma$ is the Rayleigh relaxation coefficient. In the $2 \times 2$ box model, for $0<p<p_{c}$, three solutions exist: one stable thermal mode, one unstable thermal mode and one stable haline mode; for $p>p_{c}$, only one stable haline mode exists. Here $p_{c}$ is the nondimensional critical freshwater forcing. Note the similarity between upper panel of Figure 2.2 here and Figure 5 in Huang et al. (1992).

Therefore, our scaling analysis is consistent with the above box models in the following aspects: under certain conditions, three solutions are possible, which include one stable thermal mode, one unstable thermal mode and one stable haline mode; otherwise only one stable haline mode is possible. Compared to Marotzke (1990), the criterion on $R=\alpha \Delta T / \beta \Delta S_{0}$ for the stability is different, we got an unstable solution for $1<R<4 / 3$ through the scaling argument which corresponds to the range $1<R<2$ in Marotzke (1990). As in Marotzke (1990) and Huang et al. (1992), we obtain an upper limit of the freshwater forcing (or equivalent salt flux), beyond which no thermal mode can exist.

In contrast to the box models, we include the Coriolis parameter, basin dimensions, and more importantly the two uncertain variables: diapycnal diffusivity and freshwater forcing. For a given set of parameters, the scaling analysis permits an estimate of how the thermohaline circulation will behave, which is unattainable with the box models. The role of freshwater forcing has been studied extensively in the box models, 
but the effect of diapycnal diffusivity cannot be examined due to the strong numerical diffusion intrinsic to these models.

\subsection{Description of the Numerical Model}

This study employs GFDL MOM2 (K. Bryan, 1969; Pacanowski et al. 1993; Pacanowski 1995), which is widely used as an ocean general circulation model for climate studies.

In formulating the equations of the model the Navier-Stokes equations have been modified in three important respects: the Boussinesq approximation, the hydrostatic approximation and only the large-scale motion is treated explicitly and the stresses exerted by small-scale motions are taken into account by a "turbulent viscosity" hypothesis. Molecular viscosity and conductivity are very much smaller effects and are consequently neglected.

Let

$$
\begin{aligned}
m & =\sec \varphi \\
n & =\sin \varphi \\
u & =a \dot{\lambda} / m \\
v & =a \dot{\varphi}
\end{aligned}
$$

where $a$ is the radius of the earth, $\varphi$ is latitude, and $\lambda$ is longitude. We write the advection operator such that

$$
\mathcal{L} \mu=\frac{m}{a}\left[(u \mu)_{\lambda}+(v \mu / m)_{\varphi}\right]+(w \mu)_{z},
$$

where $\mu$ is an arbitrary scalar quantity. Then the momentum equation can be written as,

$$
\begin{aligned}
u_{t}+\mathcal{L} u-2 \Omega n v-m n u v / a & =-\frac{m}{a}\left(p / \rho_{0}\right)_{\lambda}+F^{\lambda}, \\
v_{t}+\mathcal{L} v+2 \Omega n u+m n u u / a & =-\frac{1}{a}\left(p / \rho_{0}\right)_{\varphi}+F^{\varphi} .
\end{aligned}
$$

The hydrostatic relation is:

$$
\rho g=-p_{z},
$$


and the continuity equation is:

$$
w_{z}+\frac{m}{a}\left[u_{\lambda}+(v / m)_{\varphi}\right]=0
$$

There are two tracer conservation equations,

$$
\begin{aligned}
& T_{t}+\mathcal{L} T=Q \\
& S_{t}+\mathcal{L} S=\sigma .
\end{aligned}
$$

The effect of compression on the temperature is not included. The equation of state is based on an equation of the form

$$
\rho=F(T, S, p)
$$

given by Bryan and Cox (1972). The effect of pressure in this equation may be taken into account with a high degree of accuracy by substituting $-\rho_{0} g z$ for $p$. The terms $F^{\lambda}, F^{\varphi}, Q$ and $\sigma$ represent the effects of turbulent viscosity and diffusion. Let

$$
\Delta \mu=m^{2} \mu_{\lambda \lambda}+m\left(\mu_{\varphi} / m\right)_{\varphi}
$$

Then

$$
\begin{aligned}
F^{\lambda} & =A_{v} u_{z z}+\frac{A_{M}}{a^{2}}\left[\Delta u+\left(1-m^{2} n^{2}\right) u-2 n m^{2} v_{\lambda}\right] \\
F^{\varphi} & =A_{v} v_{z z}+\frac{A_{M}}{a^{2}}\left[\Delta v+\left(1-m^{2} n^{2}\right) v+2 n m^{2} u_{\lambda}\right] \\
Q & =\left(K_{T} T_{z}\right)_{z}+\frac{A_{H}}{a^{2}} \Delta T \\
\sigma & =\left(K_{S} S_{z}\right)_{z}+\frac{A_{H}}{a^{2}} \Delta S
\end{aligned}
$$

The formulation of the $F^{\lambda}, F^{\varphi}$ is in the anisotropic case, where mixing in the vertical differs from that in the horizontal. For the large-scale ocean circulation, the depth of the ocean is much smaller than the horizontal scale.

The nature of vertical mixing has not been understood very well so far. Conventionally it is assumed that $K_{T}=K_{S}$, while in this thesis, lots of study is focused on the effects of double diffusion, which means $K_{T}$ and $K_{S}$ are different, and depend on the local vertical gradients of temperature and salinity. 
We use Neumann boundary conditions on temperature and salinity at the lateral walls (no-flux condition):

$$
T_{n}, S_{n}=0
$$

where ()$_{n}$ indicates a local derivative with respect to the coordinate normal to the wall. On velocity we use Dirichlet boundary conditions (no-slip condition):

$$
u, v=0 \text {. }
$$

At the upper boundary, a rigid-lid and wind stress is imposed,

$$
\begin{aligned}
w & =0 \\
\rho_{0} A_{v}\left(u_{z}, v_{z}\right) & =\tau^{\lambda}, \tau^{\varphi}
\end{aligned}
$$

where $\tau^{\lambda}$ and $\tau^{\varphi}$ are the zonal and meridional component of the surface stress, respectively. In these runs, the effect of $\tau^{\varphi}$ is neglected. $\rho_{0}$ is the density of sea water at surface pressure and standard temperature and salinity.

At the lower boundary the very small effects of geothermal heat flow are neglected,

$$
\begin{aligned}
\left(T_{z}, S_{z}\right) & =0 \\
w(-H) & =-\frac{u(-H)}{a} m H_{\lambda}-\frac{v(-H)}{a} H_{\varphi} \\
\rho_{0} A_{v}\left(u_{z}, v_{z}\right) & =\tau_{B}^{\lambda}, \tau_{B}^{\varphi} .
\end{aligned}
$$

In this model, the bottom stress is not specified and we have no topography at the bottom, thus actually we have:

$$
\begin{aligned}
& w(-H)=0 \\
& \left(u_{z}, v_{z}\right)=0
\end{aligned}
$$

at the bottom.

This model is physically configured as a simple flat-bottomed box extending $60^{\circ}$ in longitude and from $0^{\circ}$ to $60^{\circ} \mathrm{N}$ latitude.

Resolution is $3.75^{\circ}$ in both meridional and zonal dimensions, with 15 levels in the vertical: level spacing and depths are found in Table 2.2 . 
Table 2.2: The Level Depths

\begin{tabular}{c|c|c}
\hline \hline Vertical Level & Thickness of level $(\mathrm{m})$ & Depths of $(\mathrm{T}, \mathrm{S})$ points $(\mathrm{m})$ \\
\hline 1 & 50 & 25 \\
\hline 2 & 75 & 87.5 \\
\hline 3 & 100 & 175 \\
\hline 4 & 125 & 287.5 \\
\hline 5 & 150 & 425 \\
\hline 6 & 200 & 600 \\
\hline 7 & 250 & 825 \\
\hline 8 & 300 & 1100 \\
\hline 9 & 400 & 1450 \\
\hline 10 & 450 & 1875 \\
\hline 11 & 450 & 2325 \\
\hline 12 & 450 & 2775 \\
\hline 13 & 500 & 3250 \\
\hline 14 & 500 & 3750 \\
\hline 15 & 500 & 4250 \\
\hline \hline
\end{tabular}

The horizontal and vertical momentum viscosity coefficients are $A_{h}=1.0 \times 10^{6} \mathrm{~m}^{2} \mathrm{~s}^{-1}$ and $A_{v}=20 \mathrm{~cm}^{2} s^{-1}$ respectively. For the tracer equations, the eddy transport parameterization of Gent and McWilliams (1990) and isopycnal/diapycnal mixing is used, and no background horizontal diffusion is needed. We take $K_{I S O}=K_{I T D}=$ $500 \mathrm{~m}^{2} \mathrm{~s}^{-1}$, where $K_{I S O}$ is the isopycnal diffusion coefficient and $K_{I T D}$ is the downgradient diffusivity of the isopycnal thickness. However, Weaver and Eby (1997) showed that the above scheme may cause numerical problems. These numerical problems can be reduced or eliminated if sufficient explicit (unphysical) background horizontal diffusion is added to the mixing scheme. 


\subsection{Numerical Experiments}

\subsubsection{General Sensitivity Experiments}

Scaling relation (2.12) is the basis for the scaling analysis under a variety of upper boundary conditions. Therefore it is essential to examine how robust the above relation is in numerical models. Here we investigate the sensitivity of thermohaline circulation to the Coriolis parameter and the meridional surface density difference.

For simplicity, we use the following in the numerical model described in the last section:

(a) only temperature is used to force the model;

(b) a linear density equation

$$
\rho=\rho_{0}(1-\alpha T)
$$

is used, where $\alpha=1.5 \times 10^{-4} K^{-1}$ is a constant;

(c) the sea surface temperature is relaxed to a linear distribution with latitude.

The following two groups of experiments are performed:

(1) Sensitivity to the Coriolis parameter $f=2 \Omega \sin \theta$, where $\Omega$ is the rotational rate of the earth and $\theta$ is the latitude. $\Omega$ is varied with $0.1 \Omega_{0}, 0.25 \Omega_{0}, \Omega_{0}, 2.0 \Omega_{0}, 4.0 \Omega_{0}$ where $\Omega_{0}=7.29 \times 10^{-5} \mathrm{~s}^{-1}$ is the rotational frequency of the earth. The results are plotted in Figure 2.3.

(2) Sensitivity to the north-south density (temperature) difference. Four experiments are performed, with the north-south temperature difference of $3 K, 10 K, 20 K, 100 K$ respectively. The results is plotted in Figure 2.4.

In the above two groups of parameter sensitivity experiments, the power law dependence of the meridional overturning rate on Coriolis parameter and north-south density difference highly depends on the latitude. The closer to northern and southern wall, the more the power law distorts away from those predicted by scaling analysis. In the mid-latitudes, however, the numerical experiments and scaling analysis are very consistent. The reason is that the thermal wind relation may not apply to boundary regions, where the friction must play a more significant role, and therefore the geostrophic and hydrostatic relations are not valid. 

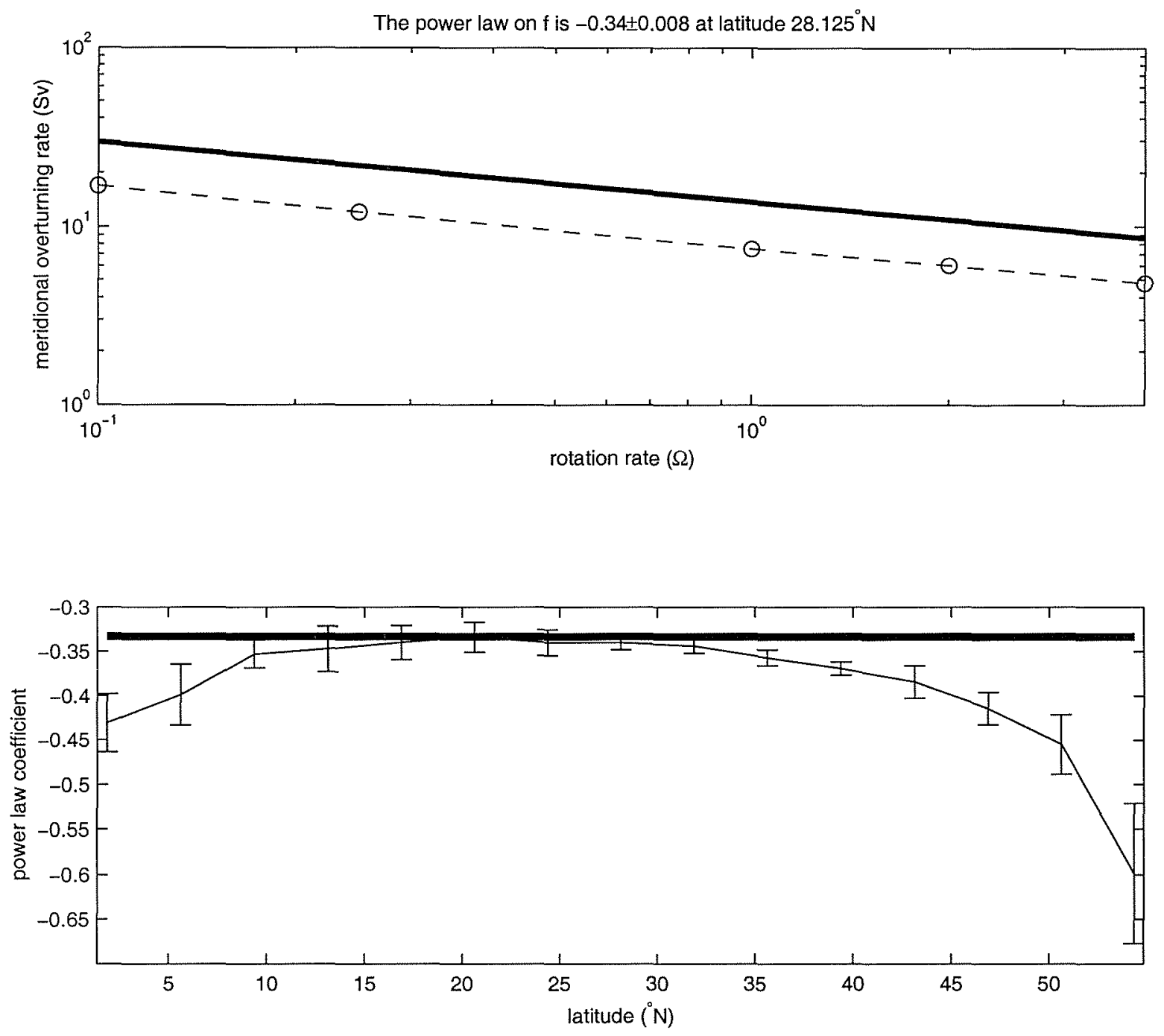

Figure 2.3: The sensitivity of the meridional overturning rate to the Coriolis parameter in numerical experiments (circles) and scaling analysis (heavy solid line). The upper panel illustrates the comparison of the meridional overturning rate at latitude $28.125^{\circ} \mathrm{N}$, and the lower panel shows the comparison of the power law dependence on Coriolis parameter at each latitude. 

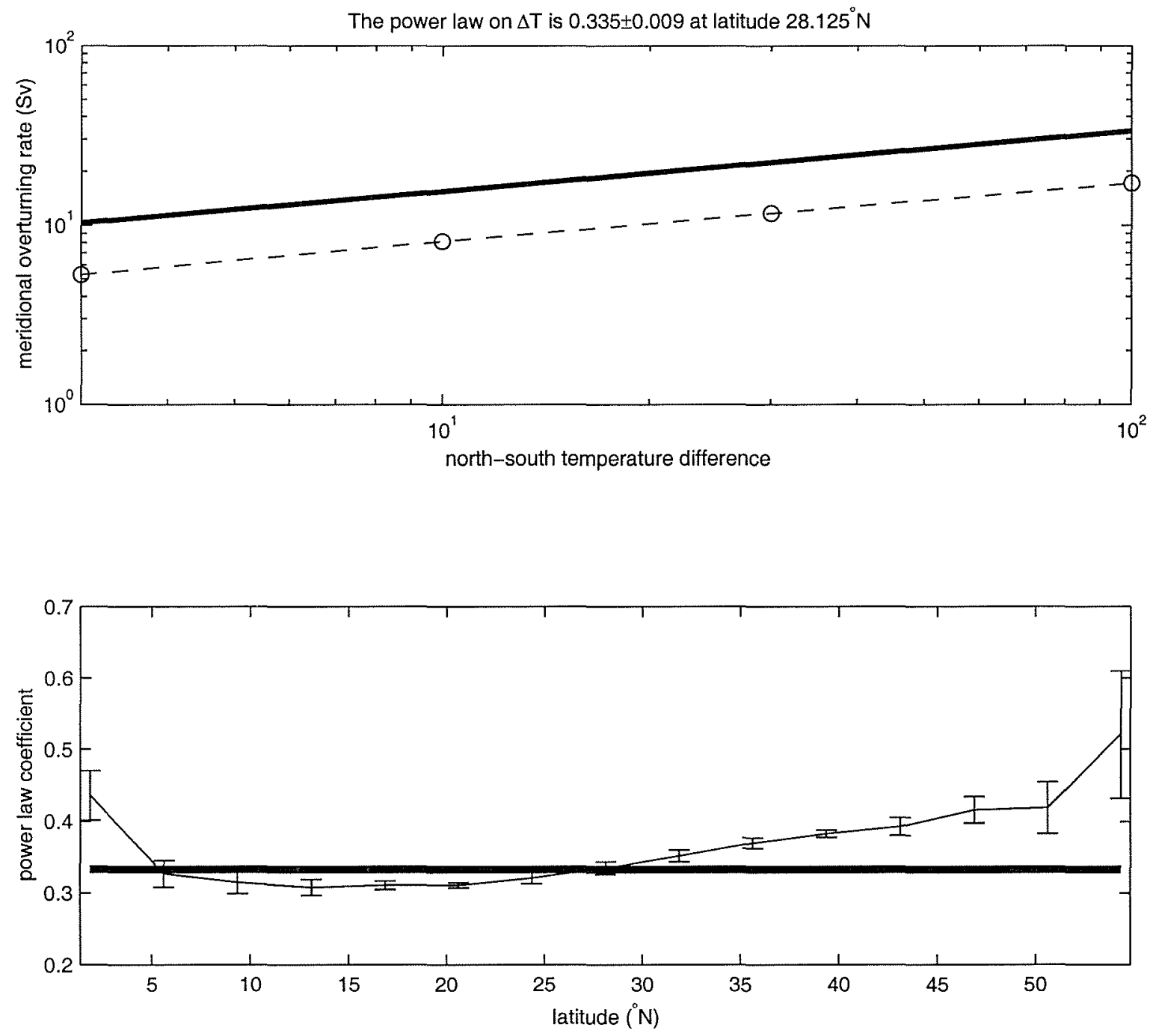

Figure 2.4: The sensitivity of the meridional overturning rate to the surface density difference in numerical experiments (circles) and scaling analysis (heavy solid line). The upper panel illustrates the comparison of the meridional overturning rate at latitude $28.125^{\circ} \mathrm{N}$, and the lower panel shows the comparison of the power law dependence on north-south density difference at each latitude. 


\subsubsection{Relaxation Boundary Conditions}

The first layer temperature and salinity are relaxed to the SST and SSS climatology (Levitus, 1982), with a relaxation time of 30 days (see Figure 2.5). Two groups of experiments were implemented, one group without any wind stress, and in the other, the zonally averaged annual climatological surface wind stress of Hellerman and Rosenstein (1983) is used. In both groups of experiments, constant diapycnal diffusivities $0.1,0.25,0.5,1.0,2.0,5.0 \mathrm{~cm}^{2} \mathrm{~s}^{-1}$ were adopted, with all other parameters fixed. The $0.1 \mathrm{~cm}^{2} \mathrm{~s}^{-1}$ experiments were run for 12,000 years, the $5.0 \mathrm{~cm}^{2} \mathrm{~s}^{-1}$ experiments were run for 4,000 years, and all others for 8,000 years. Equilibria were reached in each experiment(with the surface averaged upward heat flux oscillating less than about $\left.0.05 \mathrm{Wm}^{-2}\right)$.

The experiments with and without wind stress are consistent with the scaling argument (Figure 2.6). When the diapycnal diffusivity is very small, wind stress plays an increasingly important role in the thermohaline circulation, since the penetration depth forced by the wind stress becomes greater than that caused by buoyancy effects. In contrast, when the diapycnal diffusivity is as large as $0.5 \mathrm{~cm}^{2} \mathrm{~s}^{-1}$, the wind stress makes almost no difference to the thermohaline circulation under the relaxation boundary conditions.

When F. Bryan (1987) examined the sensitivity of the thermohaline circulation to the vertical diffusivity, he derived the two-thirds power law relation through scaling arguments, but only got an approximate one-third power law dependence in the numerical experiments. One reason is that the experiments were run only to 1,200 years, which, for most of the experiments, is not long enough to reach equilibrium. In addition, the diapycnal mixing due to the action of the horizontal diffusivity on sloping isopycnals could also affect the thermohaline circulation. Wright and Stocker (1992) used a two-dimensional (vertical-meridional) model to examine the sensitivity of the thermohaline circulation to the vertical diffusivity. For the North Atlantic ocean, they found $M \propto K^{1 / 3}$ with wind stress and $M \propto K^{1 / 2}$ without wind stress. Their model applied to the Pacific and the Indian oceans obtained $M \propto K^{2 / 3}$. Marotzke (1997) restricted all vertical mixing to the boundaries and found $M \propto K^{2 / 3}$. Here we obtain the two-thirds power law dependence in a three-dimensional OGCM with uniform diapycnal diffusivity. 

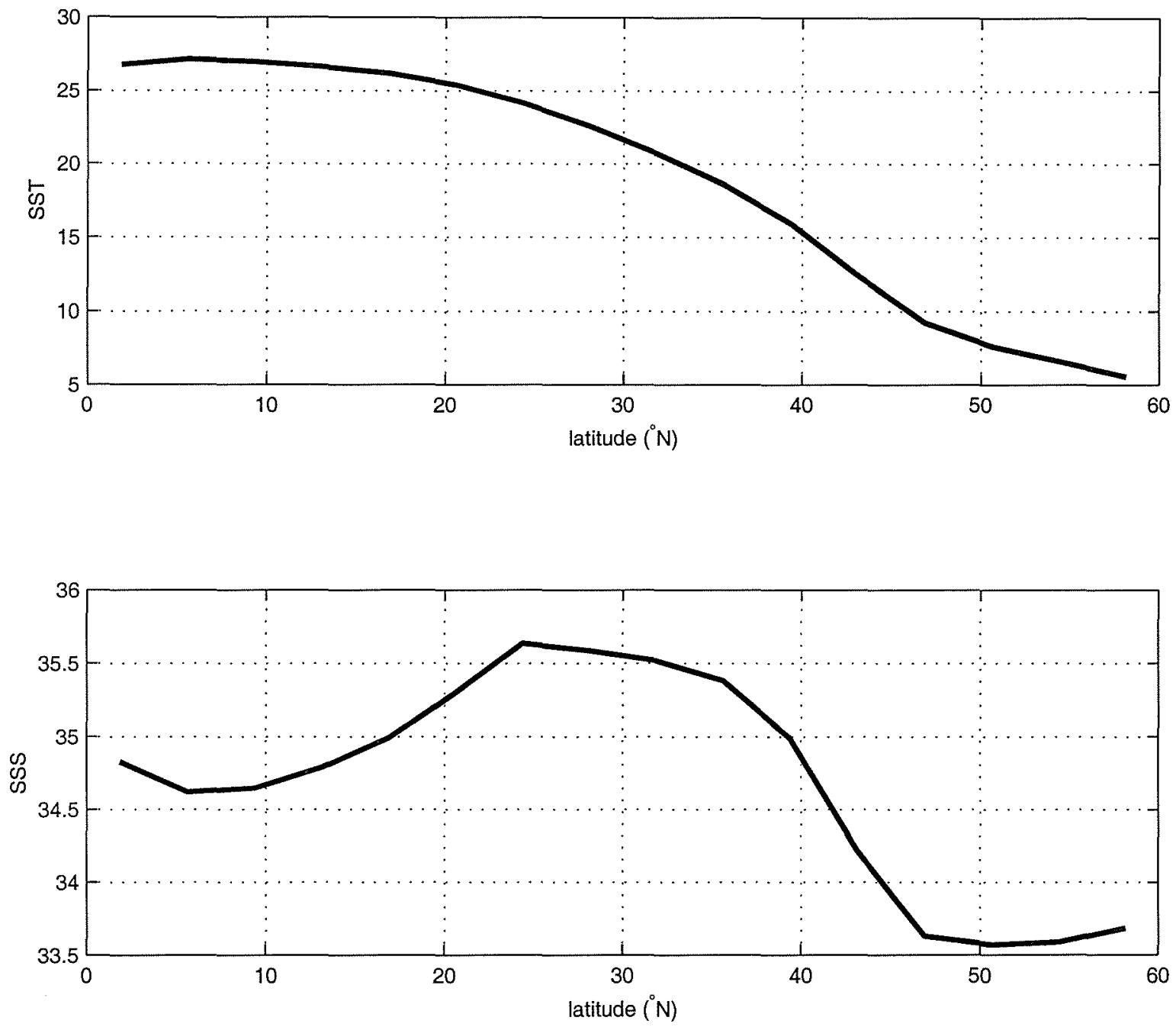

Figure 2.5: The prescribed SST and SSS fields for the experiments under RBC. Here we have approximately $\Delta T=20 K$ and $\Delta S=2 p s u$. 


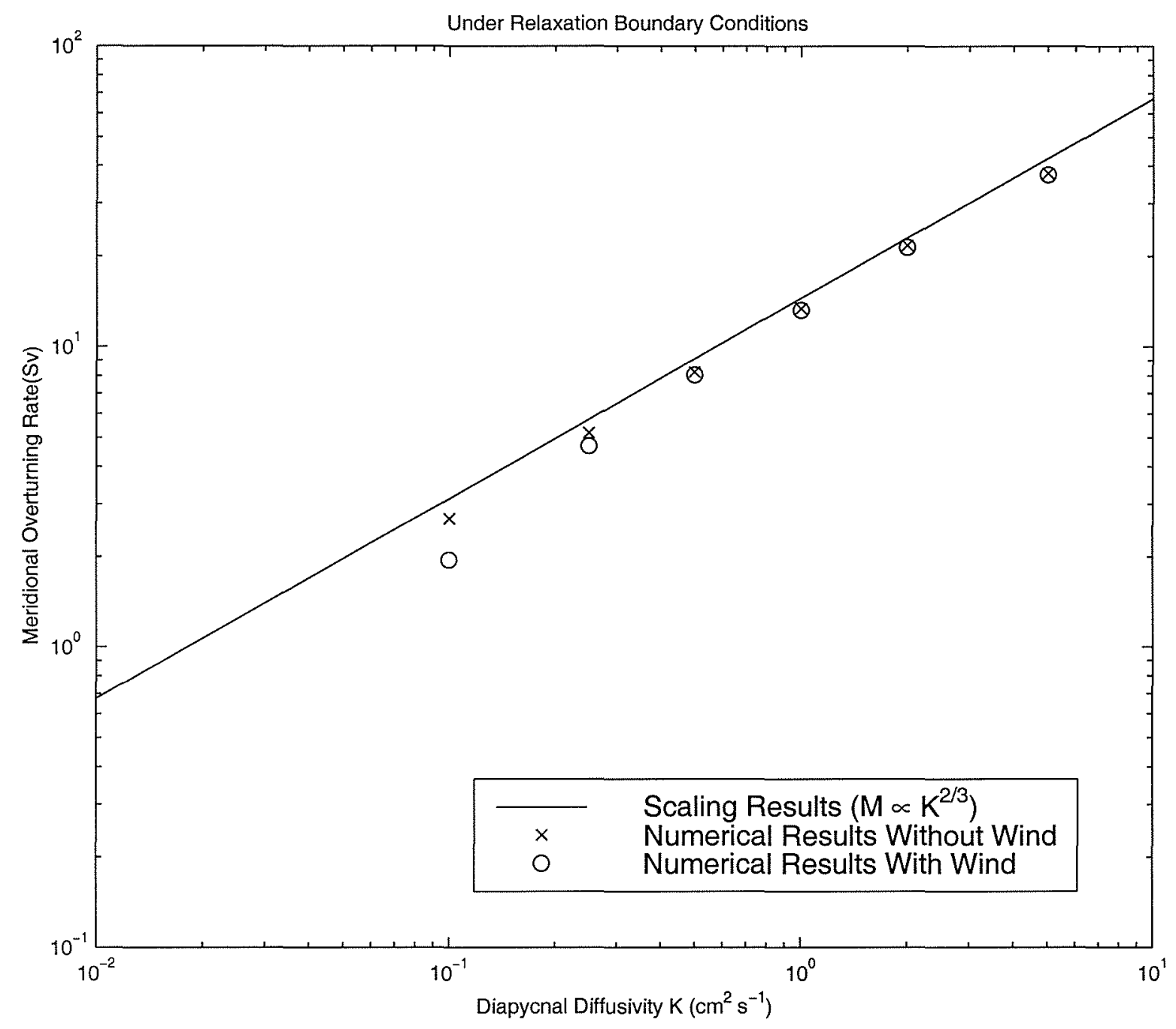

Figure 2.6: Comparison of the scaling results and numerical experiments of the meridional overturning rate under relaxation boundary conditions. The solid line represents the scaling argument results, with $\Delta S=2 \mathrm{psu}$ and other parameters the same as in (2.63), and the " $\mathrm{x}$ " and "o" represent the numerical results without and with wind stress respectively. 
The poleward heat transports in the above numerical experiments are plotted in Figure 2.7. Note that a least-square method fitting of the numerical experiments gives

$$
C_{0}=\frac{1}{3}
$$

which is used in the scaling argument given in (2.13). Using least-square estimate, the power law dependence of the maximum poleward heat transport on the diapycnal diffusivity is $0.5908 \pm 0.0029$ for the numerical experiments without wind stress, and $0.5051 \pm 0.0405$ for the case with wind stress. We argue that for the case without wind stress an approximate two-thirds power dependence given in the scaling argument is roughly obtained. For the case with wind stress, especially in the low diffusivity experiments, the Ekman cells, driven by the wind stress, can carry a portion of the total poleward heat flux. As a result, the experimental results deviate from the twothirds power, rather it is more approximated by a half power. However, Park and Bryan (1998) used the model SST, rather than the prescribed values being relaxed to, to calculate the surface density difference for the scaling analysis, and they found that the poleward heat transport obeys the two-thirds power law dependence on the diapycnal diffusivity very closely. In general, the underlying dynamics of the power dependence of the maximum poleward heat transport on diapycnal diffusivity, especially the role of wind stress, is less clear than that for the meridional overturning rate.

One significant difference between the model formulation here and the earlier work (F. Bryan, 1987; Wright and Stocker, 1992; Marotzke, 1997) is the inclusion of the Gent and McWilliams (1990) isopycnal tracer mixing scheme, which eliminates the false diapycnal fluxes caused by horizontal diffusion, especially in places where the slope of the isopycnals is large, like western boundary current and convection regions. We believe that this explains why the present model estimates of meridional overturning so closely obey the two-thirds power law dependence on the vertical diffusivity derived in the scaling analysis. 


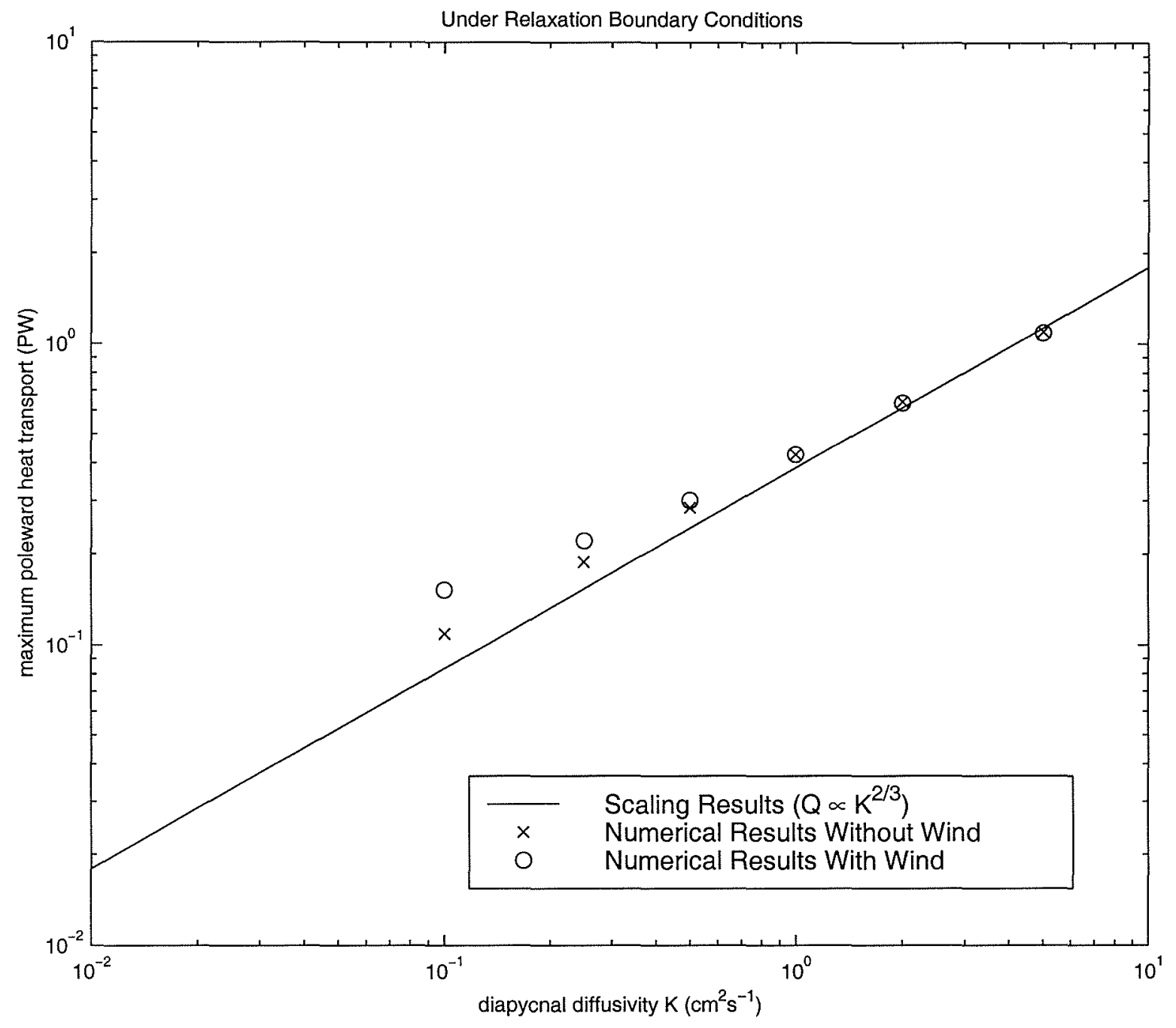

Figure 2.7: Comparison of the scaling results and numerical experiments of the poleward heat transport under relaxation boundary conditions. The solid line represents the scaling argument results, with parameters the same as in Figure 2.6, and the " $\mathrm{x}$ " and "o" represent the numerical results without and with wind stress respectively. 


\subsubsection{Flux Boundary Conditions}

Here we use flux boundary conditions for both temperature and salinity. The heat flux and freshwater flux are quasi-linear functions of latitude:

$$
\begin{aligned}
Q & =\frac{Q_{0}}{\cos \phi}\left(1-\frac{2 \phi}{\phi_{n}}\right) \\
e-p & =\frac{W_{0}}{\cos \phi}\left(1-\frac{2 \phi}{\phi_{n}}\right)
\end{aligned}
$$

then the maximum and minimum of $Q, e-p$ are $Q_{0}, W_{0}$ (at $\left.\phi=0\right)$ and $-2 Q_{0},-2 W_{0}$ (at $\phi=\phi_{n}=60^{\circ}$ ) respectively (see Figure 2.8 where $W_{0}=1 \mathrm{~m}_{\text {year }}{ }^{-1}$ ), therefore, we have

$$
\begin{aligned}
& Q=1.5 Q_{0} \\
& E=1.5 W_{0}
\end{aligned}
$$

to represent the magnitude of heat flux and freshwater flux in the scaling analysis respectively.

The sensitivity of the meridional overturning rate to the diapycnal diffusivity is plotted in Figure 2.9. The solid line represents the $1 / 2$ power-law result from scaling analysis and the triangles are the numerical experiment results in GFDL MOM2. We can see that the numerical experiments are consistent with the scaling analysis under flux boundary conditions. Note that the above scaling power law has been investigated and confirmed in Huang and Chou (1994) when only freshwater forcing is applied.

Under flux boundary conditions, the strength of the thermohaline circulation is determined by the internal vertical mixing, as well as the external forcing like heat flux and freshwater flux. Sensitivity of the meridional overturning to the magnitude of the heat flux is investigated and the results are plotted in Figure 2.10. The horizontal axis is the strength of heat flux $Q$ defined in (2.108). Again, the solid line is the result from scaling analysis and triangles represent the results from numerical experiments. They are very consistent. To a close approximation, the scaling analysis provides a simple way to predict the behaviour of the numerical model. 


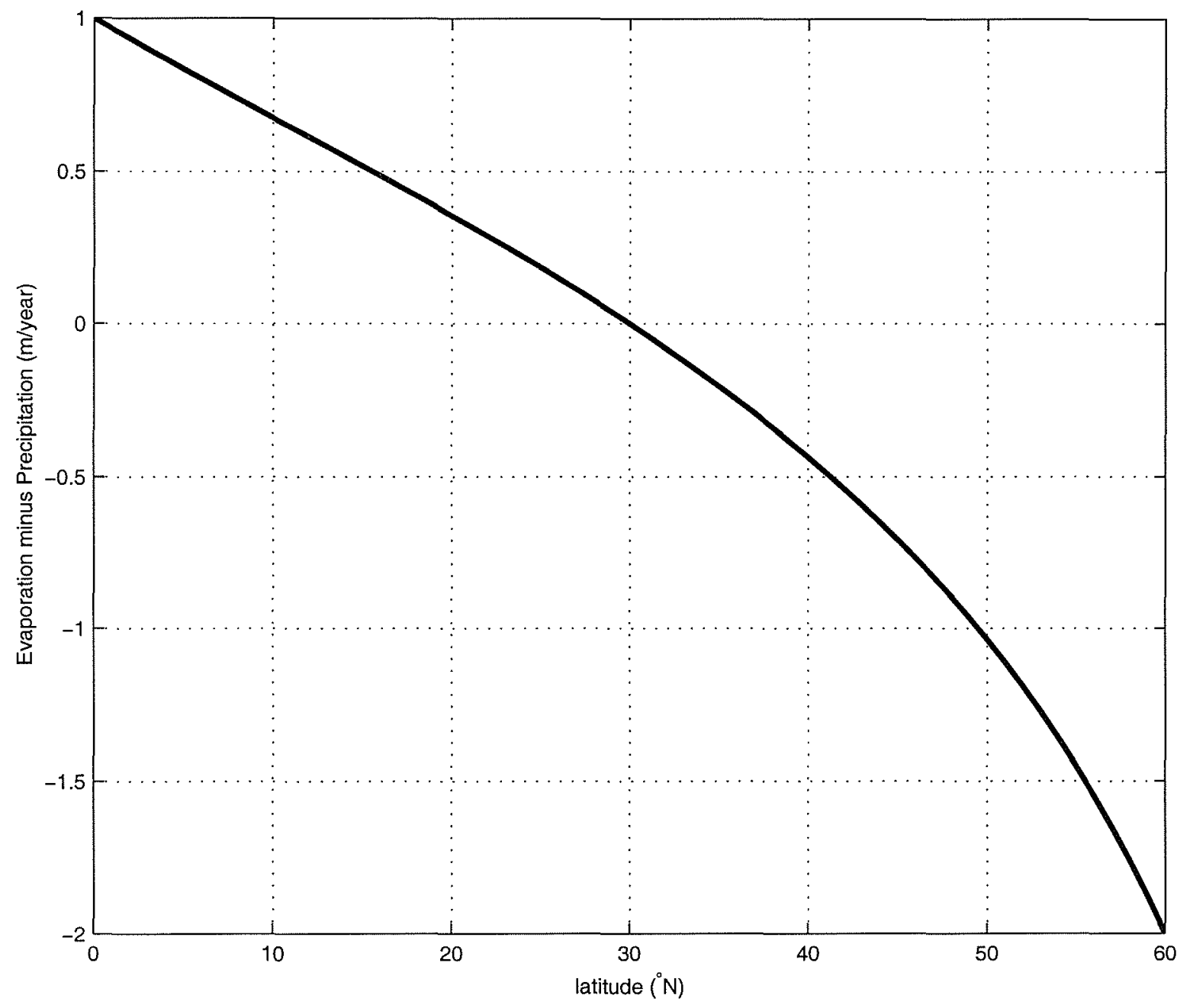

Figure 2.8: The profile of Evaporation minus Precipitation with latitude when $W_{0}=$ $1 m$ year $^{-1}$. 


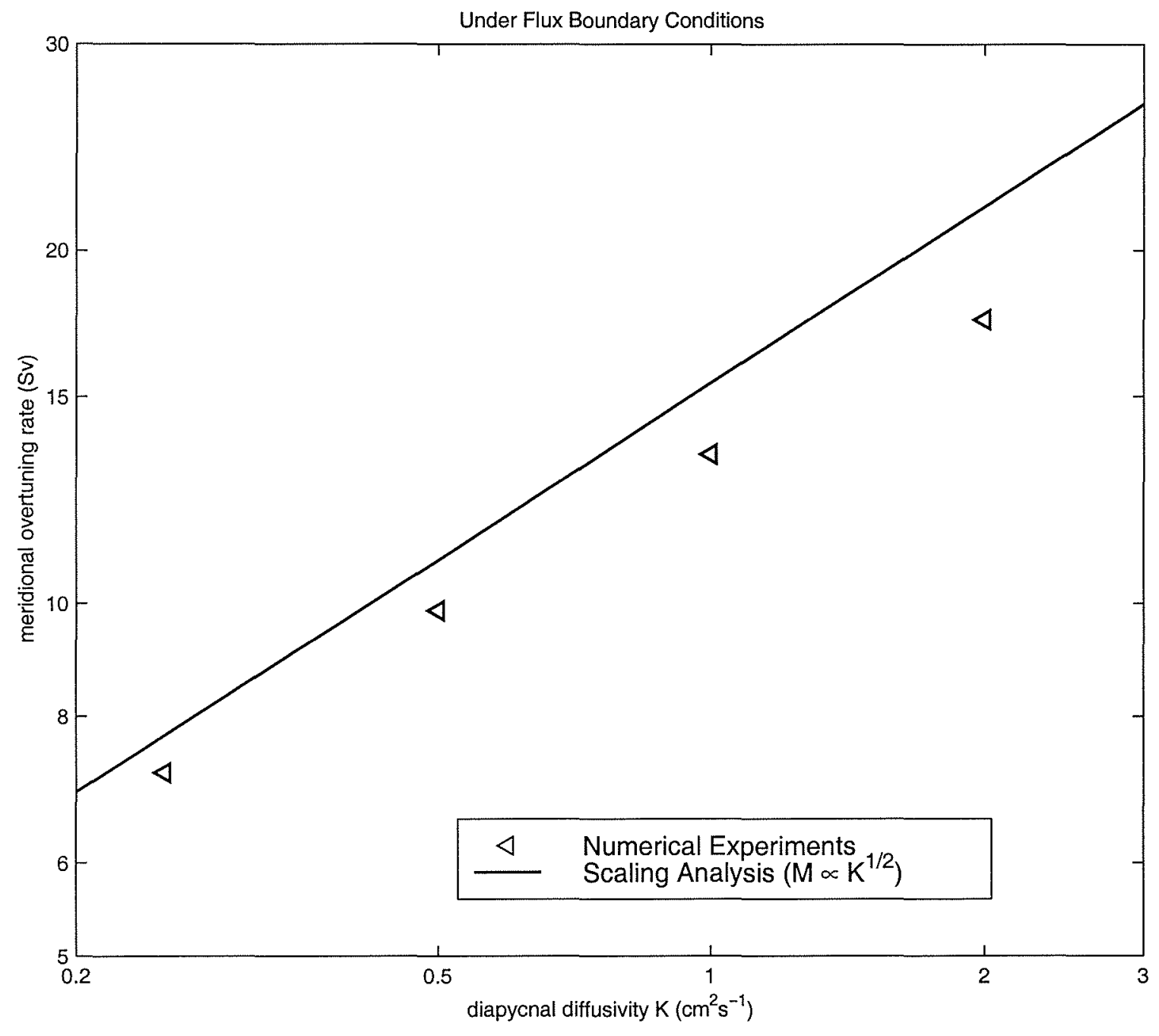

Figure 2.9: Sensitivity of the meridional overturning rate to the vertical diffusivity in numerical experiments (triangles) and scaling analysis (heavy solid line) under flux boundary conditions. 


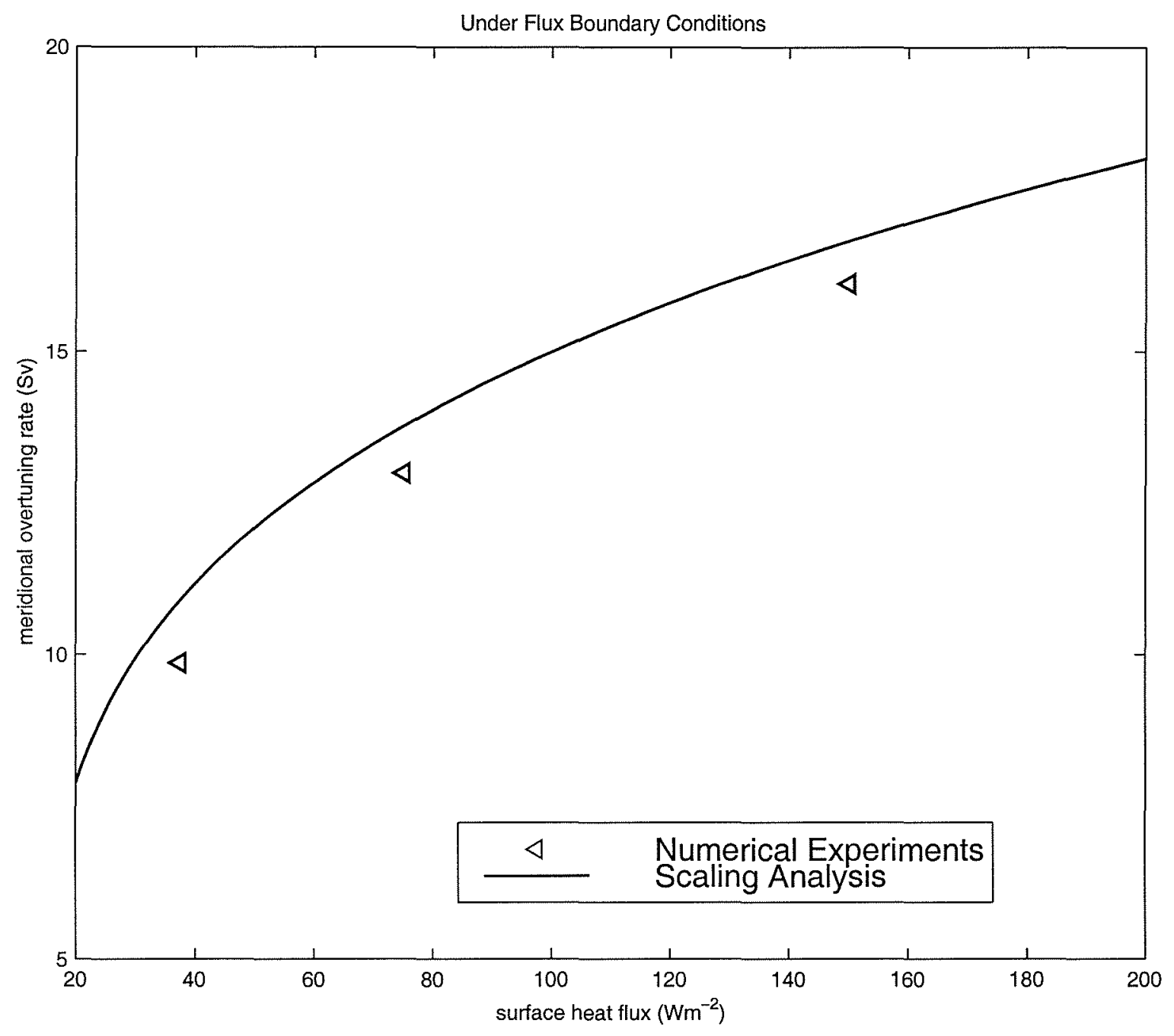

Figure 2.10: Sensitivity of the meridional overturning rate to the surface heat flux in numerical experiments (triangles) and scaling analysis (heavy solid line) under flux boundary conditions. 


\subsubsection{Mixed Boundary Conditions}

Here we use a relaxation condition for temperature (same as in the upper panel of Figure 2.5) and a virtual salt flux boundary condition for the salinity as (2.107). Therefore, we have

$$
E=1.5 W_{0}
$$

to represent the magnitude of the freshwater forcing, as defined in the scaling argument. This $E$ will be used in the scaling estimate for comparison with the numerical experiments. When $E>0$, the freshwater forcing opposes the thermal forcing; when $E<0$, polar water is salty, deep water can form only close to the northern wall, and we obtain only the thermal mode.

Three groups of quasi-equilibrium experiments are shown, with $K=0.3,0.5,1.0$ $\mathrm{cm}^{2} \mathrm{~s}^{-1}$ respectively. In order to find the bifurcation point of the freshwater forcing, we first run the model to an equilibrium with $E \leq 0$. From there we increase $E$ very slowly ( 0.1 or $0.05 \mathrm{~m}_{\text {year }}{ }^{-1}$ per thousand years). Due to the slow rate of change of the forcing, the model remains in quasi-equilibrium and we can see the quasi-equilibrium response of the model to the different freshwater forcings while the thermal forcing is basically fixed. The results are plotted in Figure $2.11-2.13$ respectively.

For each group of experiments, there is an upper limit of $E$, beyond which the thermal mode does not exist. If we continue to increase the freshwater forcing past this point, the thermohaline circulation will stay in the haline mode. When we decrease $E$ starting from a haline mode, the ocean stays in the mode until "flushing", a phenomenon peculiar to the haline mode, occurs. In the numerical runs, the model cannot reach equilibrium in the haline mode. Over a long period, the polar deep ocean becomes warmer and saltier. With cold and fresh water overlying the warm and salty deep water in the polar basin, an instability sets in and very strong convection occurs, which releases the heat accumulated for hundreds or thousands of years (Marotzke 1990; Huang, 1994). However, even with flushing we can see that under certain conditions, there are two modes possible: one thermal mode and one haline mode, which is consistent with the scaling and stability analysis.

In order to gain confidence in the quasi-equilibrium effects displayed in the above experiments, we conducted a series of experiments, where $K=0.5 \mathrm{~cm}^{2} \mathrm{~s}^{-1}$ and with 


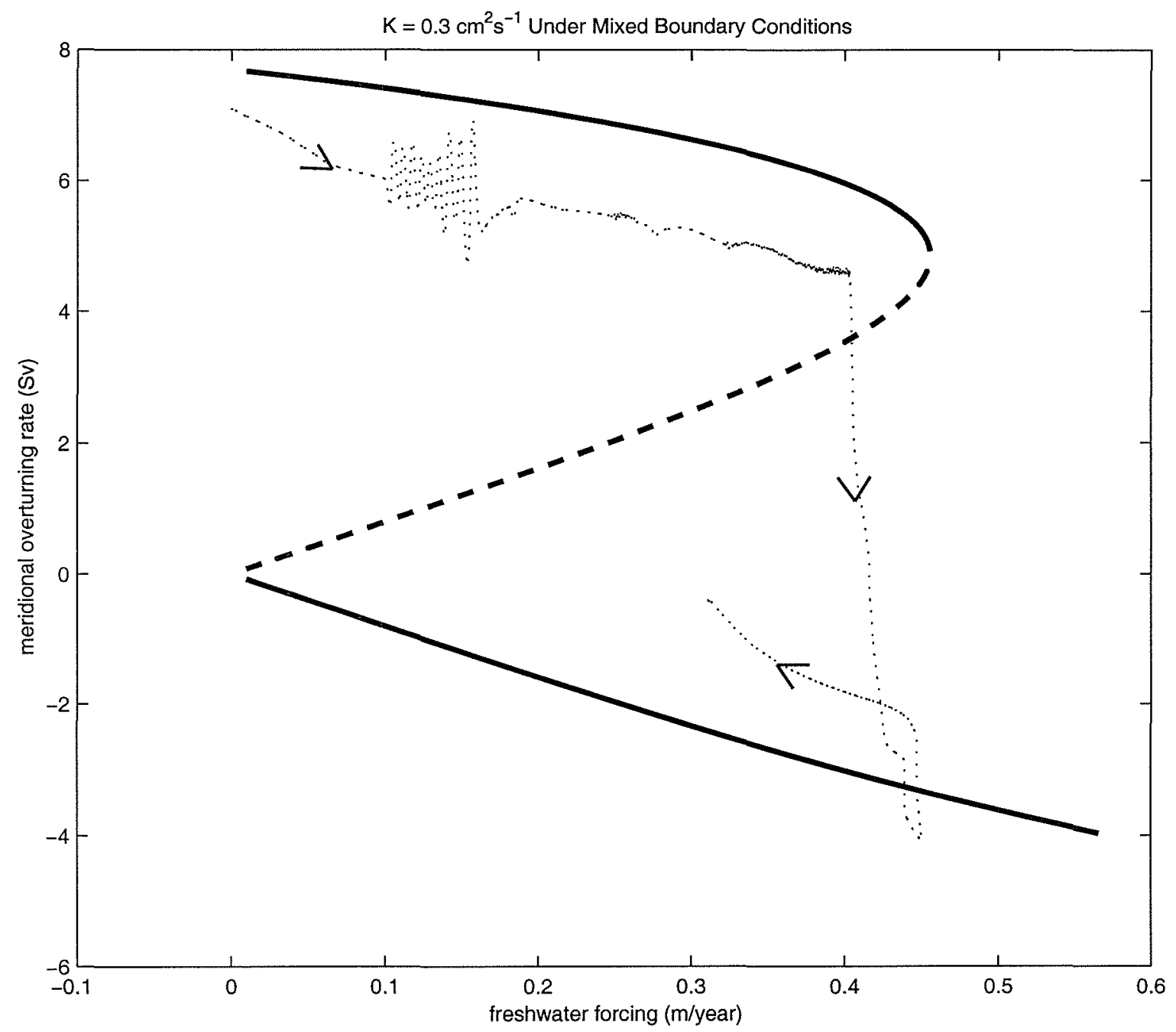

Figure 2.11: Comparison of the scaling results and the quasi-equilibrium numerical experiments under mixed boundary conditions with $K=0.3 \mathrm{~cm}^{2} \mathrm{~s}^{-1}$. Here the solid line represents the results from scaling analysis and the dotted line is the quasiequilibrium response of the numerical model to the very slowing varying freshwater forcing. Arrow directions represent the freshwater change with time in the process of integration. 


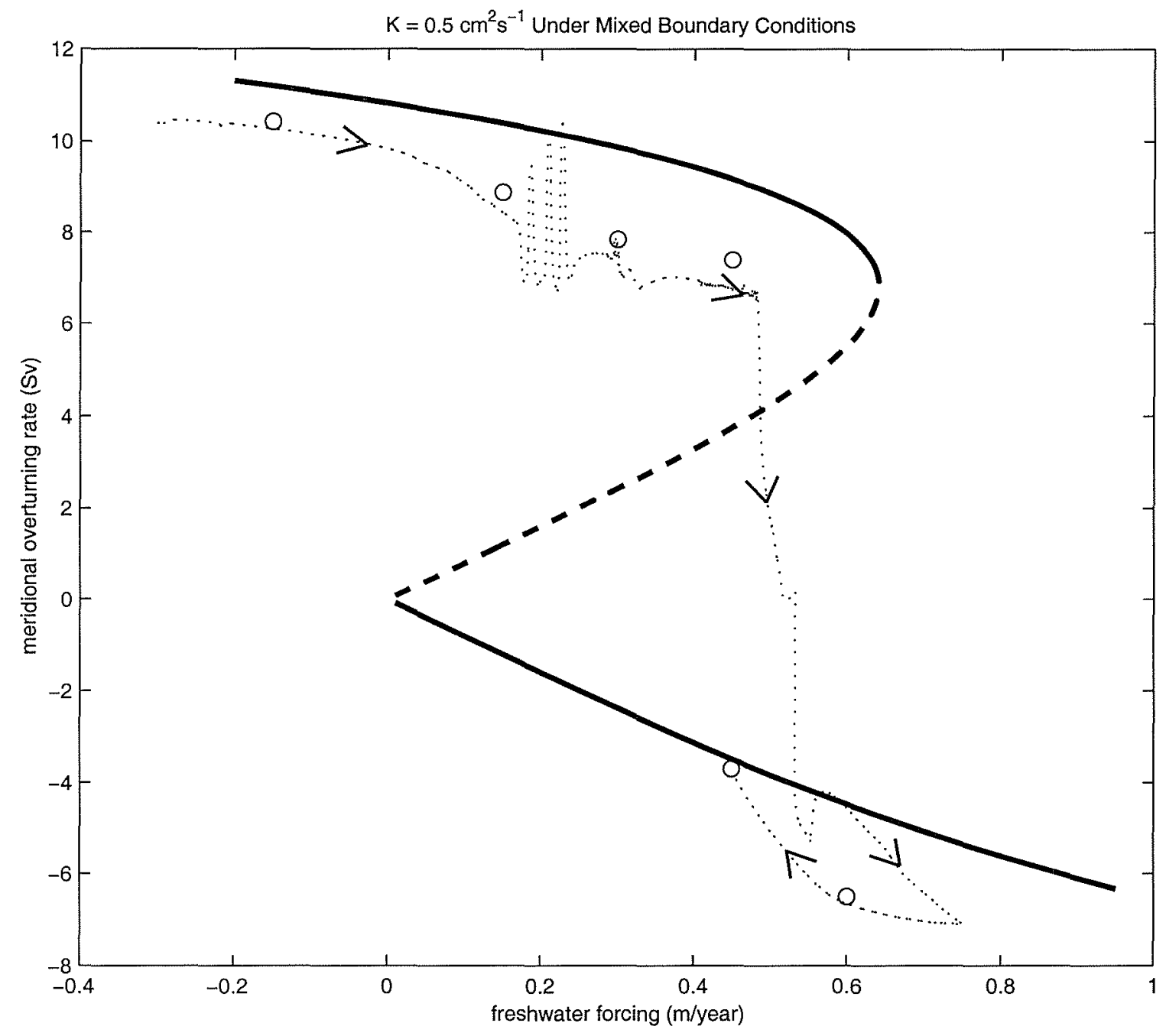

Figure 2.12: Comparison of the scaling results and the numerical experiments under mixed boundary conditions with $K=0.5 \mathrm{~cm}^{2} \mathrm{~s}^{-1}$. Here the thick lines represents the results from scaling analysis, the line with arrows is the quasi-equilibrium response of the numerical model to the very slowly changing freshwater forcing and the circles represent the single numerical experiments for different $E$ forcing. Arrow directions represent the freshwater change with time in the process of integration. 


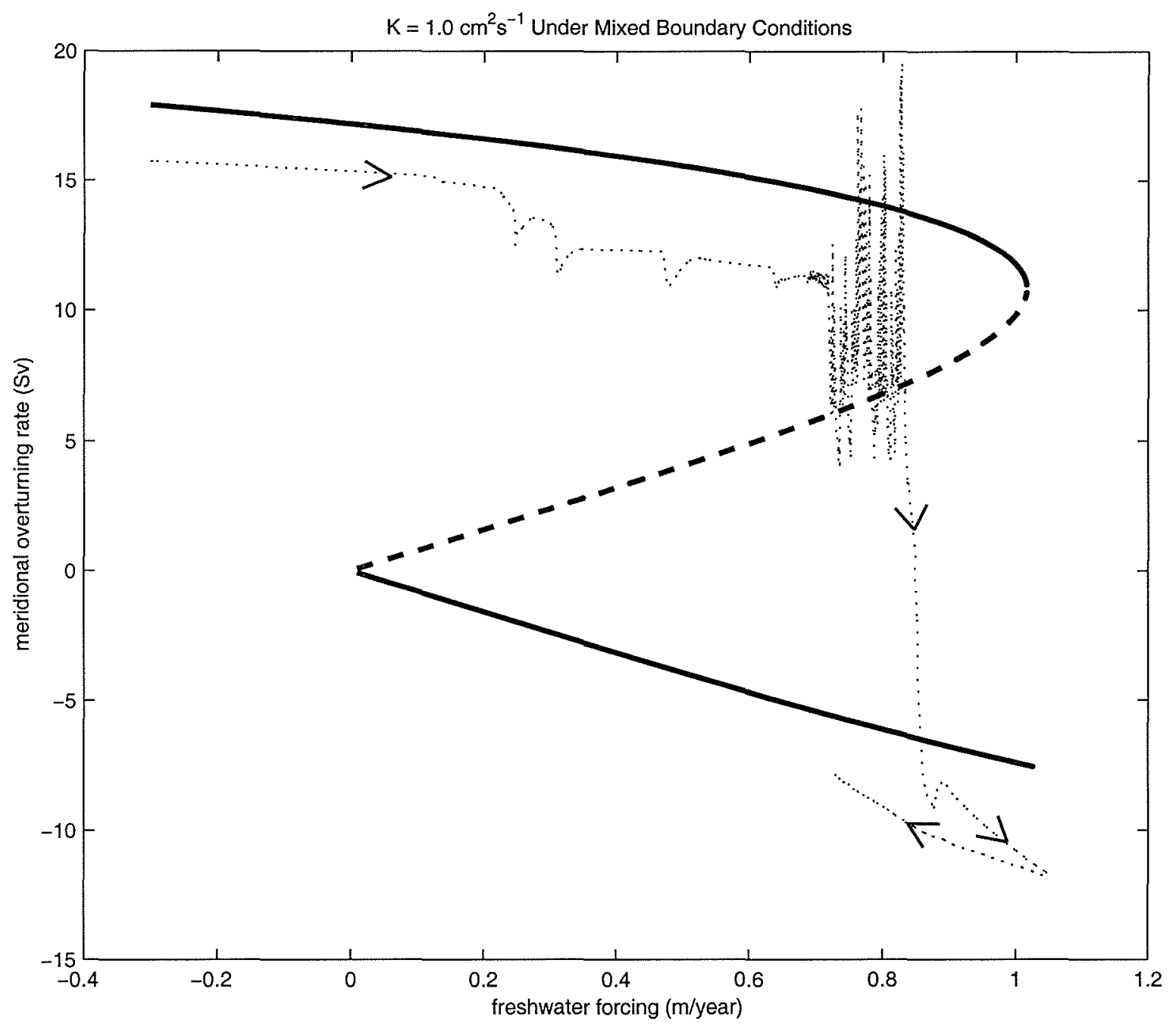

Figure 2.13: Comparison of the scaling results and the quasi-equilibrium numerical experiments under mixed boundary conditions with $K=1.0 \mathrm{~cm}^{2} \mathrm{~s}^{-1}$. Here the solid line represents the results from scaling analysis and the dotted line is the quasiequilibrium response of the numerical model to the very slowing changing freshwater forcing. Arrow directions represent the freshwater change with time in the process of integration. 
different values of $E$. We start the first experiment from $E=-0.15 \mathrm{~m}$ year ${ }^{-1}$ and an initially homogeneous state. After 4000 years of integration, we obtained quasiequilibrium. We start the next experiment from this final state with another $E$ and run it to another equilibrium. When the $E$ is large enough, the upper limit of the freshwater forcing is passed and the ocean enters a haline mode. Since there is no steady equilibrium for a haline mode, we run the model for at least one flushing period, i.e., we stop the experiment in the haline mode after at least one flushing event. Then we begin to decrease $E$ to a specified value and continue our experiments. The results are plotted in Figure 2.12 (the "o" points in the figure). We can see that they are close to the quasi-equilibrium experiments and we obtained two modes for a value of $E=0.45$ m year $^{-1}$.

Through the above experiments, we find that for each $K$, there is an upper limit on $E$ for the existence of a stable thermal mode, below which two modes are possible, as predicted by the scaling analysis. We plot the different critical $E$ versus diapycnal diffusivity in Figure 2.14. The numerical experiments are consistent with the scaling argument, though quantitatively there is a difference. The linear equation of state assumed in the scaling argument could cause such a distortion since the full state equation used in the numerical models is highly nonlinear (the thermal expansion coefficient $\alpha$ is a strong function of temperature). Convection plays an essential role in deep water formation and the top-to-bottom density difference is important for convection. Thus it is crucial to determine the role of temperature and salinity in the vertical density distribution in the convection region. For the thermal mode, convection occurs in the polar region, where the temperature is low and thus $\alpha$ is small (about $1.0 \times 10^{-4} \mathrm{~K}^{-1}$ at $4^{\circ} \mathrm{C}$ ); since we use a universal value $2.0 \times 10^{-4} \mathrm{~K}^{-1}$ in the scaling, the magnitude of the meridional overturning for the thermal mode, as well as the magnitude of the critical freshwater flux, are overestimated.

Weaver et al. (1993) investigated the effect of freshwater fluxes on the behavior of the thermohaline circulation. They concluded that the freshwater forcing is the dominant factor in determining the model's stability and internal variability. Increasing the relative importance of freshwater fluxes versus thermal forcing led to, in turn, one stable steady state of the model, two stable states, one stable, and one unstable equilibrium, or no stable steady states. If the freshwater forcing is sufficiently strong, self-sustained oscillations exist in the deep-water formation rate, which last 


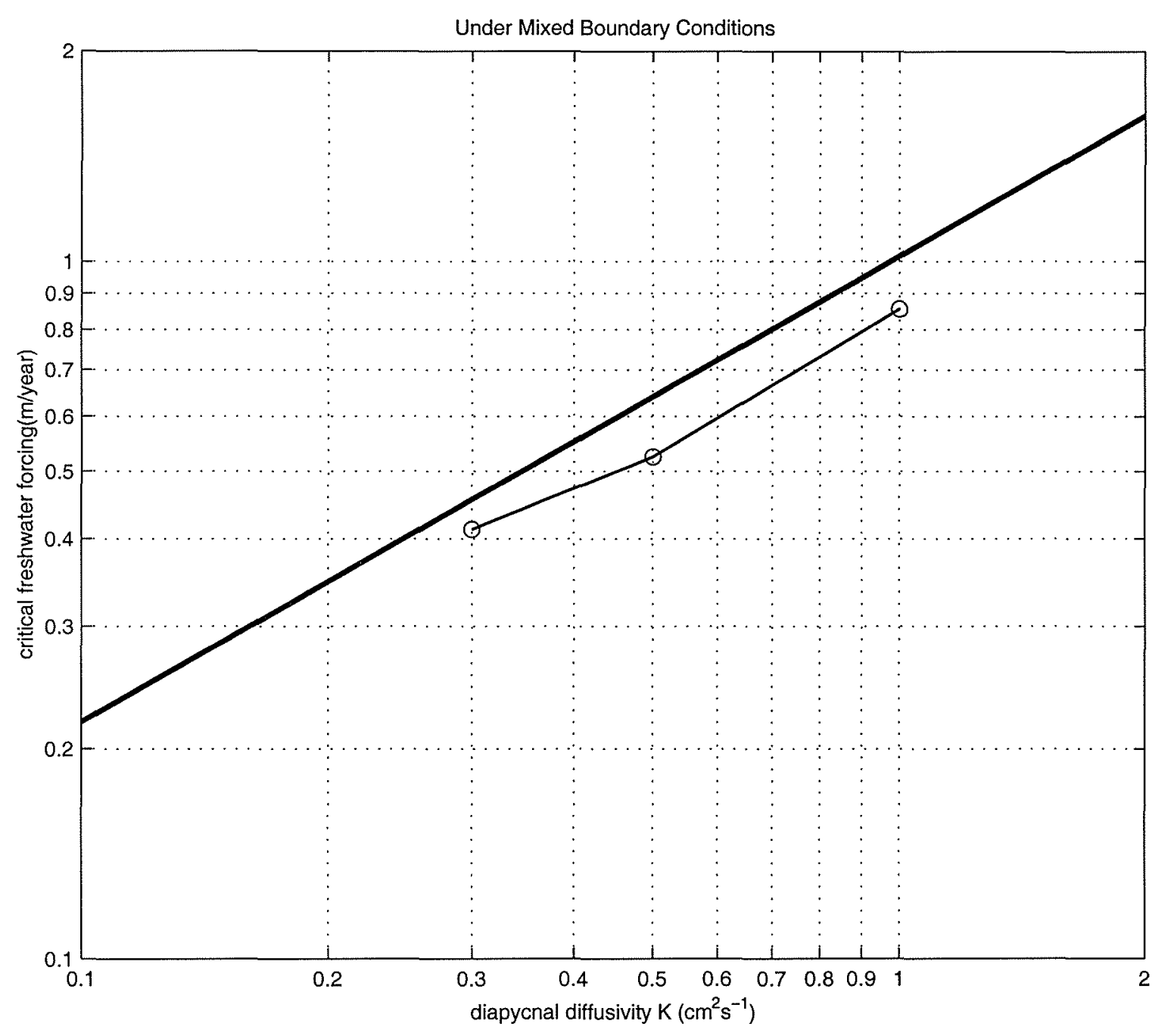

Figure 2.14: Comparison of the scaling results and the numerical experiments for the critical freshwater versus vertical eddy diffusivity. The solid line is for scaling results $\left(E_{c} \propto K^{2 / 3}\right)$ and the circles are from the quasi-equilibrium numerical experiments. 
thousands of years. We find a transition from a multi-equilibria region to a single haline mode when we increase the magnitude of the freshwater forcing, and this is basically consistent with our scaling argument. However, in contrast to Weaver et al. (1993), the criterion determining the behavior of the thermohaline circulation is a nondimensional number defined by (2.42), which includes not only the freshwater forcing versus thermal forcing, but also the effect of diapycnal diffusivity and the dimensions of the basin. For a given basin, the diapycnal diffusivity also determines the behavior of the thermohaline circulation. Weaver et al. (1993) investigated the use of different vertical diffusivities. In one experiment a value of $K=0.5 \mathrm{~cm}^{2} \mathrm{~s}^{-1}$ was set throughout the water column, in another $K$ was made to increase from $0.3 \mathrm{~cm}^{2} \mathrm{~s}^{-1}$ at the surface to $1.3 \mathrm{~cm}^{2} \mathrm{~s}^{-1}$ at the bottom of the model ocean. No major differences were observed. However, from Cummins et al. (1990), we deduce that the value of diapycnal diffusivity in the thermocline is more important than that in the deep ocean. As the above two values of vertical diffusivity are very close, it is not surprising that Weaver et al. (1993) saw little difference, as far as the behavior of the thermohaline circulation is concerned, even though the T-S structure in the deep ocean could be very different. As suggested by the scaling analysis and the numerical experiments presented here, not only the freshwater forcing, but also the diapycnal diffusivity play important roles in determining the stability and variability of the thermohaline circulation under mixed boundary conditions.

Rahmstorf (1995) investigated the sensitivity of the North Atlantic thermohaline circulation to the input of fresh water using a global ocean circulation model coupled to a simplified atmospheric model. In his experiments, moderate changes in freshwater input induced transitions between different equilibrium states, leading to substantial changes in regional climate. His experiments provide a map of the equilibrium states and bifurcation points of the Atlantic thermohaline circulation, as a function of freshwater flux. The saddle-node bifurcation first described by Stommel (1961) is confirmed in Rahmstorf (1995)'s experiments. Beyond the bifurcation point, the North Atlantic Deep Water (NADW) circulation cannot be sustained. Below the bifurcation point, at least two states are found: one is characterized by absence of NADW, one with NADW. Qualitatively, the experiments in Rahmstorf (1995) are consistent with the scaling analysis in this article. In addition, given the sensitivity of the thermohaline circulation to the diapycnal diffusivity deduced in the present 
scaling argument, it is highly likely that the bifurcation point in the coupled GCM will be sensitive to the diapycnal (vertical) diffusivity used.

Also, we can define the poleward heat transport as in (2.13) for the multiple solutions we obtained under the mixed boundary conditions. For the stable thermal mode, we choose $C_{0}=1 / 3$ as given in (2.105); for the haline mode, however, $C_{0}=1 / 3$ seems to overestimate the poleward heat transport, because unlike the thermal mode in which the convected water can sink to the deep ocean (3000 or 4000 meters) and the deep water is nearly homogeneous, the convection can only reach to $1000 \mathrm{~m}$ in the haline mode. Thus the low-latitude formed "deep" water is a thermocline water mass and the temperature difference between these waters and the surface return flow is greatly reduced. For simplicity, we choose $C_{0}=1 / 6$ in the haline mode for the scaling analysis.

For $K=0.5 \mathrm{~cm}^{2} \mathrm{~s}^{-1}$, the results are plotted in Figure 2.15. Similar to that under relaxation conditions, the results from numerical experiments are consistent with the scaling analysis. It is obvious that the stable thermal mode transports heat poleward far more efficiently than the haline mode.

Another type of quasi-equilibrium experiment is to fix the freshwater forcing and then change the magnitude of the vertical mixing very slowly with time. We use a fixed freshwater forcing of $W_{0}=0.30 \mathrm{~m}$ year $^{-1}\left(E=0.45 \mathrm{~m}\right.$ year $\left.{ }^{-1}\right)$ and start the experiment with $K=1.5 \mathrm{~cm}^{2} \mathrm{~s}^{-1}$. First we run the model to an equilibrium and then we decrease the magnitude of the vertical mixing with time at a very slow rate $\left(0.2 \mathrm{~cm}^{2} \mathrm{~s}^{-1}\right.$ per thousand years), so that the model responds in quasi-equilibrium. The change of the meridional overturning rate with the changing vertical mixing is plotted in Figure 2.16. When the vertical diffusivity is larger than $1.0 \mathrm{~cm}^{2} \mathrm{~s}^{-1}$, the numerical experiment follows the scaling analysis closely. When the vertical diffusivity decreases, first there is an oscillation in the magnitude of the meridional overturning and then a chaotic behavior when we further decrease the vertical diffusivity. At about $0.5 \mathrm{~cm}^{2} \mathrm{~s}^{-1}$, the thermal mode drops dramatically to nearly zero, where no thermal mode can be sustained. 


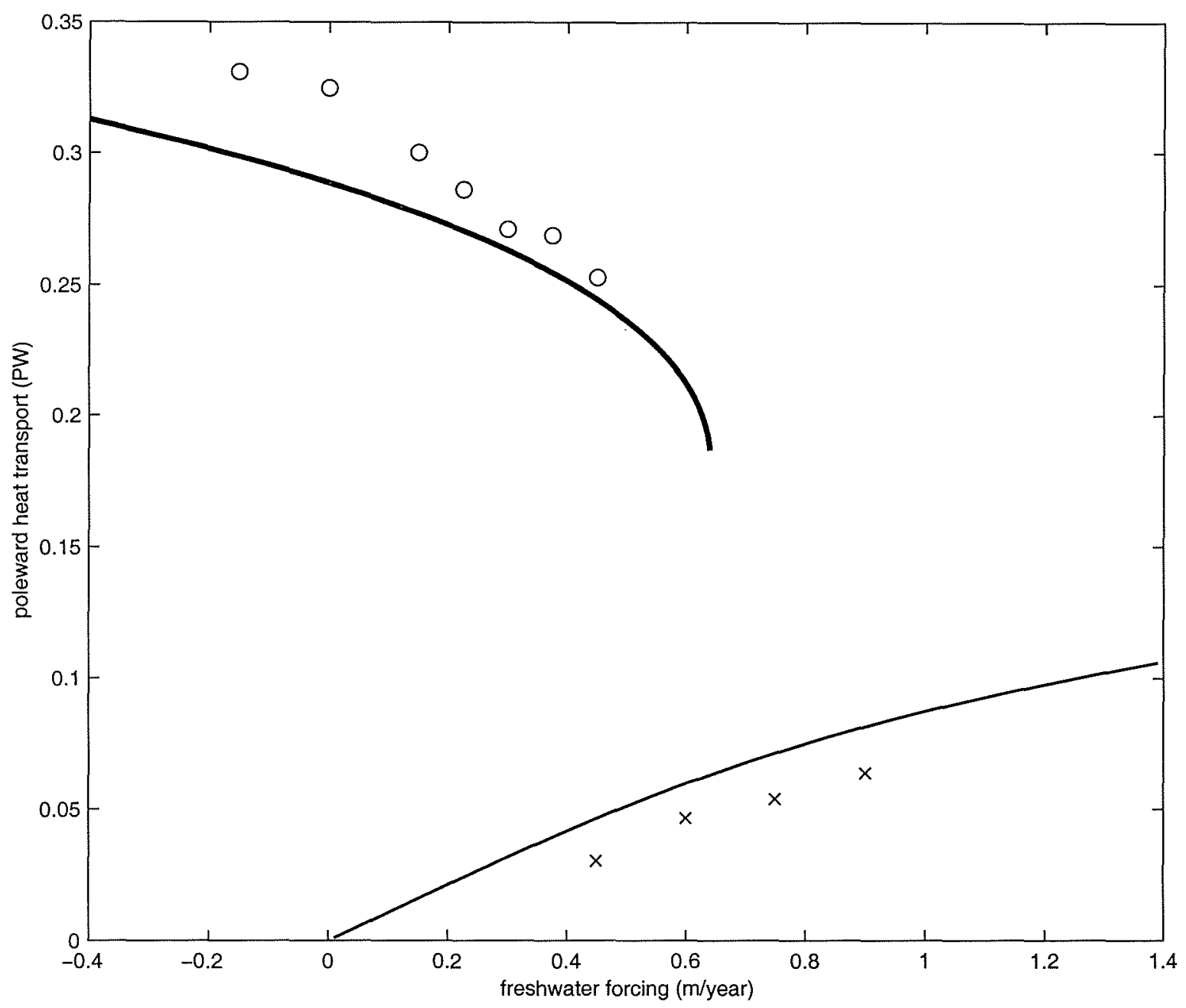

Figure 2.15: Comparison of the scaling results and the numerical experiments for the poleward heat transport under mixed boundary conditions. The solid lines are for scaling results (heavy line for the stable thermal mode and thin line for the haline mode), and "o" are from the numerical experiments in the thermal mode and " $\mathrm{x}$ " are for numerical experiments in the haline mode. For all the numerical experiments and the scaling argument, $K=0.5 \mathrm{~cm}^{2} \mathrm{~s}^{-1}$. 


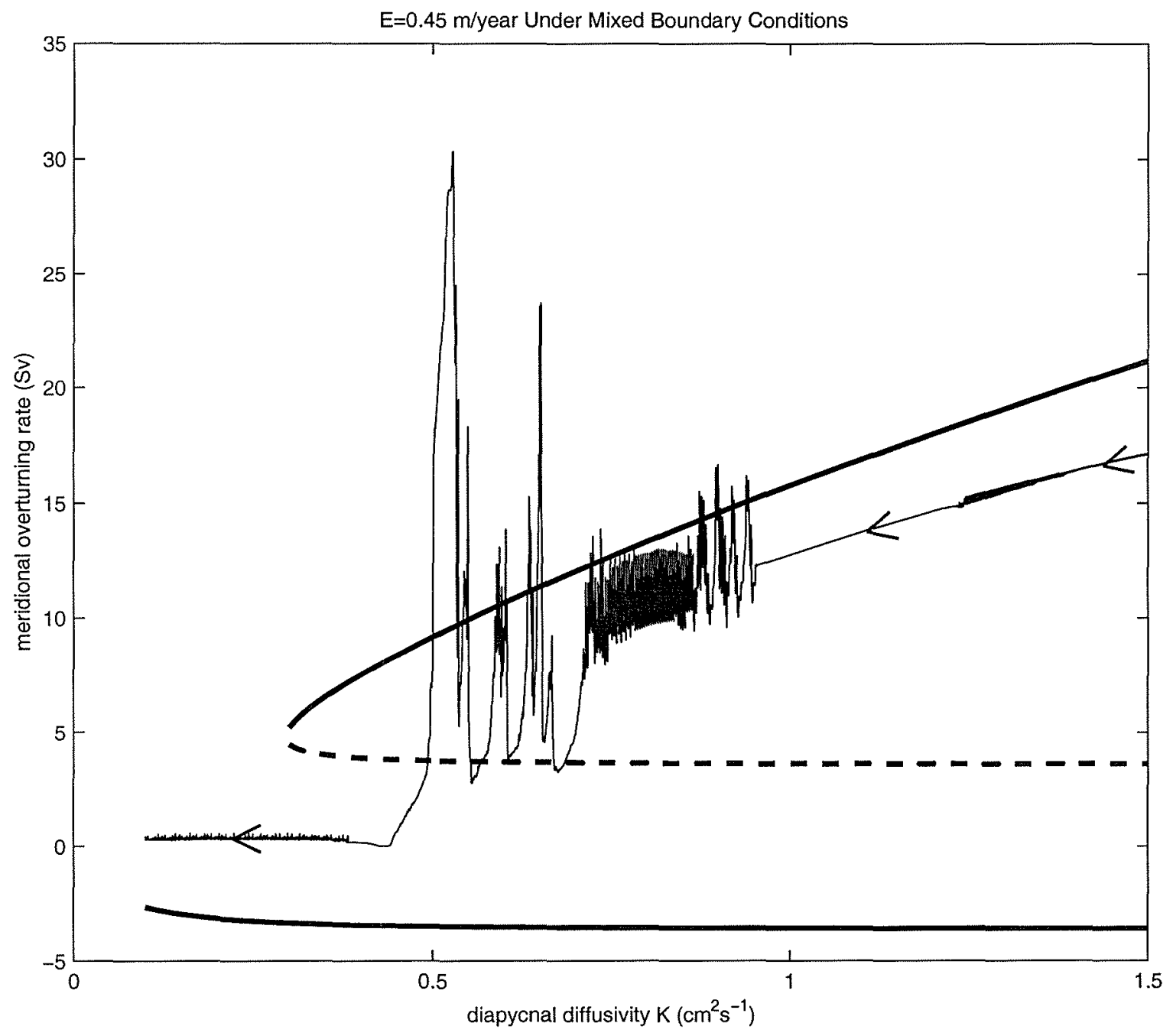

Figure 2.16: Comparison of the scaling results and the numerical experiments for the meridional overturning rate under mixed boundary conditions. For all the numerical experiments and the scaling argument, $E=0.45 \mathrm{~m}$ year $^{-1}$. Arrow directions represent the freshwater change with time in the process of integration. 


\subsection{Discussion and Conclusions}

From the scaling argument, we conclude that the thermohaline circulation behaves differently under three types of upper boundary conditions. Under "relaxation" boundary conditions or "flux" boundary conditions, there is only one equilibrium. Under "mixed" boundary conditions, multiple equilibria are possible. The states formed under mixed boundary conditions can be distinguished by a simple relation between the diapycnal diffusivity and the hydrologic forcing for a given thermal forcing. When $K>C E^{3 / 2}$, there are two thermal modes and one haline mode. When $K<C E^{3 / 2}$, there is only one haline mode. In addition, through stability analysis, we find that of the two thermal modes arising when $K>C E^{3 / 2}$, only one is a stable solution. The haline mode solution is always stable.

In the numerical experiments, a two-thirds power law dependence of meridional overturning on diapycnal diffusivity is obtained under the relaxation boundary conditions. Under mixed boundary conditions, there is an upper limit on the freshwater forcing for a given diapycnal diffusivity beyond which only a haline mode is found. Below that limit we have two modes possible: one thermal mode and one haline mode. The dependence of the critical $E$ on the diapycnal diffusivity also obeys a two-thirds power law given by the scaling argument. Similar scaling dependence is obtained for the poleward heat transport.

The scaling analysis and the numerical experiments are consistent for three types of upper boundary conditions. Thus, it is reasonable to deduce the lowest order solution using the simple scaling relation. More importantly, in contrast to the box models, we have included the Coriolis parameter, diapycnal diffusivity and freshwater forcing (for mixed boundary conditions only) in the scaling argument. Under the more physical "mixed boundary conditions", the sensitivity of the thermohaline circulation to two very uncertain variables: diapycnal diffusivity and freshwater forcing, is defined by an algebraic relation. The importance of $E$ has been previously discussed by Weaver et al. (1993) and others, while the significance of the magnitude of the diapycnal diffusivity to the stability of the thermohaline circulation has not received much attention yet. 
Through scaling analysis and simple numerical experiments, the north-south temperature difference, the magnitude of the freshwater forcing and the diapycnal diffusivity are found to be the most important factors in determining the stability and variability of the thermohaline circulation, of which the latter two are not well known so far. This requires us to develop a much better understanding of these two still challenging fields. The diapycnal (vertical) mixing processes have been under increased observational investigation recently. Ledwell et al. (1993) used a tracer release experiment to estimate a diapycnal diffusivity of $0.1-0.15 \mathrm{~cm}^{2} \mathrm{~s}^{-1}$ in the upper thermocline, which agrees with the small diffusivity estimates for internal wave processes (Gregg, 1989; Polzin et al., 1995). In contrast, Toole et al. (1994) and Polzin et al. (1997) find that the diapycnal diffusivity in the abyss is far from uniform as traditionally assumed. Instead, strong vertical mixing above rough topography can exceed $10 \mathrm{~cm}^{2} \mathrm{~s}^{-1}$, which is two orders of magnitude larger than the thermocline diffusivity found by Ledwell et al. (1993). Our analysis indicates that an improved knowledge of both diapycnal mixing and hydrologic forcing is necessary to understand the stability of the thermohaline circulation. 


\section{Chapter 3}

\section{Parameterization of}

\section{Double-diffusive Processes and}

\section{Scaling Analysis}

\subsection{Introduction}

Double-diffusive convection can occur in a stably stratified environment where the vertical density gradient due to either temperature or salinity is destabilizing. For the case with warm, salty water on top of cold, fresh water, salt fingering occurs; for the case with cold, fresh water on top of warm, salty water, diffusive layering (Turner, 1973; Stern, 1975; Schmitt, 1994) appears. Double diffusive mixing, occurring because of the two orders of magnitude difference in the molecular diffusivities of heat and salt, is driven by the release of potential energy of the destabilizing component, which has the larger buoyancy flux. A significant fraction of the released energy is used to move the stable component upward. However, the total density flux is downward, so the overall potential energy of the water column is reduced. As a result, doublediffusive processes can be self-energized through the change in the mean potential energy. In the case of ordinary turbulent mixing, however, the potential energy of 
water column is increased, thus external energy sources such as internal waves or strong means shears are required.

As introduced in Chapter 1, double-diffusive processes are widespread in the ocean. Research through theory, laboratory experiments and observations indicate that doublediffusion plays a significant role in vertical mixing, which is a dominant factor for the thermohaline circulation. Most numerical models ignore the effects of doublediffusion, except Gargett and Holloway (1992) proposed a very simple representation of double-diffusion in OGCMs. Here we propose a more accurate parameterization of double-diffusive processes in Section 3.2, and we estimate its impacts on thermohaline circulation in Section 3.3 by scaling analysis under different boundary conditions. A summary is given in Section 3.4.

\subsection{Parameterization of Double Diffusion}

In traditional numerical models diapycnal mixing in stably stratified region of the model ocean is parameterized in terms of the same constant diffusivity for both heat and salt. In this study we will divide the stably stratified regions into three subregions:

(a) salt fingering regime,

(b) diffusive layering regime and

(c) non-double-diffusive regime.

Note that the convective adjustment scheme for regions of unstable stratification remains the same as in the normal MOM2 code.

\subsubsection{Parameterization of Salt Fingering}

The extensive observational, laboratory and theoretical evidence that fingers are most intense when the density ratio is less than about 2 leads us to propose a parameterization that is dependent on $R_{\rho}$. Schmitt (1981) has argued that the constancy of the 
large scale density ratio observed in the main thermocline can be explained by this dependence, and the greater transfer rate for salt. In the modeling below we adopt a parameterization similar to his [and to the form inferred by Kunze (1990), though reduced in amplitude to conform with recent observations].

We apply the parameterization of Schmitt (1981):

$$
\begin{aligned}
K_{S} & =\frac{K^{*}}{1+\left(R_{\rho} / R_{c}\right)^{n}}+K^{\infty}, \\
K_{T} & =\frac{0.7 K^{*}}{R_{\rho}\left[1+\left(R_{\rho} / R_{c}\right)^{n}\right]}+K^{\infty},
\end{aligned}
$$

where $K_{S}$ and $K_{T}$ are the diapycnal eddy diffusivities for salinity and temperature respectively. $K^{\infty}$ is the assumed constant diapycnal diffusivity due to other mixing processes unrelated to double diffusion, such as internal wave breaking. $R_{c}$ is the critical density ratio above which the diapycnal mixing due to salt fingering drops dramatically. A value of 0.7 is used for the heat/salt buoyancy flux ratio due to salt fingers. $K^{*}$ is the maximum diapycnal diffusivity due to salt fingers. We have chosen a more modest value than originally proposed by Schmitt (1981), reflecting improved understanding of fluxes in thermohaline staircases observed in the C-SALT program (Schmitt, 1988). The exponent $n$ is an index to control the reduction of $K_{T}, K_{S}$ with increasing $R_{\rho}$.

In numerical models, vertical gradients of temperature and salinity, $T_{z}$ and $S_{z}$, are not well simulated, compared to the oceanic observations. The simulation is especially poor in the deep ocean, where the vertical gradients of temperature and salinity in low-resolution numerical models are very small and often noisy. On the other hand, most field observations of double diffusion are limited to the upper ocean, and it is not clear whether double diffusion can play any significant role at great depth where both the vertical gradients of temperature and salinity are small. Thus, we have introduced an additional constraint that double diffusion can occur only if the magnitude of the vertical temperature gradient is larger than a critical value:

$$
\left|T_{z}\right|>T_{z, c} .
$$




\subsubsection{Parameterization of Diffusive Layering}

Kelley (1984) discussed a parameterization for the diffusive layering process, in which he applied the laboratory-derived double-diffusive flux laws to oceanic data. The formulation is given as follows:

$$
\begin{aligned}
& K_{T}=C R_{a}^{1 / 3} k_{t} \\
& K_{S}=R_{F} R_{\rho} K_{T}
\end{aligned}
$$

where

$$
\begin{aligned}
C & =0.00859 \exp \left(4.6 e^{-0.54\left(R_{\rho}^{-1}-1\right)}\right), \\
R_{a} & =0.25 \times 10^{9} R_{\rho}^{-1.1} .
\end{aligned}
$$

and $k_{t}$ is the molecular diffusivity of heat, $R_{F}$ is the buoyancy flux ratio defined as

$$
R_{F}=\frac{\beta F_{S}}{\alpha F_{T}}
$$

in which $F_{S}, F_{T}$ are upward flux of salt and heat respectively. Huppert's(1971) formulation for $R_{F}$ was used by Kelley (1984):

$$
\begin{array}{rr}
R_{F}=1.85-0.85 / R_{\rho} & 0.5 \leq R_{\rho}<1.0 \\
R_{F}=0.15 & R_{\rho}<0.5,
\end{array}
$$

Roughly speaking, $K_{S} \simeq K_{T} \simeq 1.0 \mathrm{~cm}^{2} \mathrm{~s}^{-1}$ when $R_{\rho} \rightarrow 1.0 . K_{S}$ and $K_{T}$ decrease approximately as $R_{\rho}^{4}$ and $R_{\rho}^{2}$, respectively, over the range $0.1 \leq R_{\rho} \leq 1.0$.

Furthermore, Kelley (1990) refined his formulation by giving a new empirical formulation of $C\left(R_{\rho}\right)$ and $R_{F}$ :

$$
\begin{aligned}
C & =0.0032 e^{4.8 R_{\rho}^{0.72}} \\
R_{F} & =\frac{1 / R_{\rho}+1.4\left(1 / R_{\rho}-1\right)^{3 / 2}}{1+14\left(1 / R_{\rho}-1\right)^{3 / 2}} .
\end{aligned}
$$

Similar to the case of salt fingering, $K_{T}$ and $K_{S}$ are different, and they depend on the density ratio. However, The diapycnal diffusivity suggested by Kelley $(1984,1990)$ was rather weak at lower density ratio, since $K_{S}$ fell below the molecular diffusivity 
for heat $\left(1.4 \times 10^{-3} \mathrm{~cm}^{2} \mathrm{~s}^{-1}\right)$ when $R_{\rho}<0.25$. Diffusivities this small can cause computational difficulty in a numerical model. On the other hand, given the role of non-double-diffusive processes in the diapycnal mixing (Muench et al., 1990), a background diffusivity is also needed in the region of diffusive layering. Thus we include $K^{\infty}$ in the parameterization for diffusive layering:

$$
\begin{aligned}
K_{T} & =C R_{a}^{1 / 3} k_{t}+K^{\infty}, \\
K_{S} & =C R_{F} R_{\rho} R_{a}^{1 / 3} k_{t}+K^{\infty}
\end{aligned}
$$

where $C$ and $R_{F}$ are given in $(3.11-3.12)$ and $R_{a}$ defined in (3.7). Also, the restraint (3.3) is also applied in the diffusive layering case. Note, however, the definition of density ratio in this paper is inverse to that in Kelley $(1984,1990)$, in order to deal consistently with both forms of double diffusion. Note that this formulation is quite conservative compared to that of Fedorov (1988) or Muench et al. (1990), giving weaker fluxes when $R_{\rho}$ is close to 1 .

\subsubsection{Non-double-diffusive Process}

Away from the regions discussed above, we use a constant background diapycnal mixing rate for both tracers, to represent internal wave induced mixing.

$$
K_{T}=K_{S}=K^{\infty}
$$

In these runs, a value of $K^{\infty}=0.3 \mathrm{~cm}^{2} \mathrm{~s}^{-1}$ is used. This is higher than that obtained by Ledwell et al. (1993), but is necessary for computational efficiency.

\subsection{Scaling Analysis of the Impacts of Double Dif- fusion on the Thermohaline Circulation}

The sinking limb of the thermohaline circulation is driven by buoyancy losses at the air-sea interface; the return limb involves a balance between upward buoyancy advection and downward buoyancy diffusion in the ocean interior. However, in the GFDL 
MOM2, density is a diagnostic variable, thus the diapycnal eddy diffusivity of density, $K_{\rho}$, does not appear in the model explicitly. Instead, $K_{\rho}$ is defined diagnostically as a function of the density ratio, assuming a locally linear equation of state applies to the mixing between adjacent layers. An expression equivalent to that given in Gargett and Holloway (1992) is readily obtained:

$$
K_{\rho}=\frac{R_{\rho} K_{T}-K_{S}}{R_{\rho}-1} .
$$

The change of diffusivities of temperature, salinity and density with the density ratio is shown in Figure 3.1, where we take the double-diffusive parameters as

$$
\begin{array}{r}
K^{*}=1.0 \mathrm{~cm}^{2} \mathrm{~s}^{-1}, K^{\infty}=0.3 \mathrm{~cm}^{2} \mathrm{~s}^{-1}, \\
R_{c}=1.6, n=6, T_{z, c}=2.5 \times 10^{-4}{ }^{\circ} \mathrm{Cm}^{-1}
\end{array}
$$

Because heat and salt mixing rates are different, the equivalent buoyancy diffusivity varies greatly from one region to another (Figure 3.1):

(a) In the weak double-diffusive regime $\left(R_{\rho}<0.3\right.$ or $\left.R_{\rho}>3.0\right), K_{\rho}$ is close to its limit, given by the constant turbulent mixing coefficient, $K^{\infty}$.

(b) In the moderate double diffusion regime $\left(0.3<R_{\rho}<0.76\right.$ or $\left.1.56<R_{\rho}<3.0\right)$, $K_{\rho}$ is reduced, so the mixing of buoyancy is less efficient.

(c) In the strong double diffusion regime $\left(0.76<R_{\rho}<1.56\right)$, the sign of $K_{\rho}$ is reversed. Thus, upgradient buoyancy diffusion appears because the buoyancy flux due to the destabilizing component is larger than that of the stabilizing component. Given the change on the diapycnal diffusivity of density by double-diffusive processes, we can conclude that the thermohaline circulation will change accordingly, as discussed in Chapter 2. In addition, there are several free parameters in the parameterization of salt fingering phenomenon, and thus it is desirable if we can analyze how sensitive the thermohaline circulation to the above parameters, and below we will discuss that under two kinds of boundary conditions. 


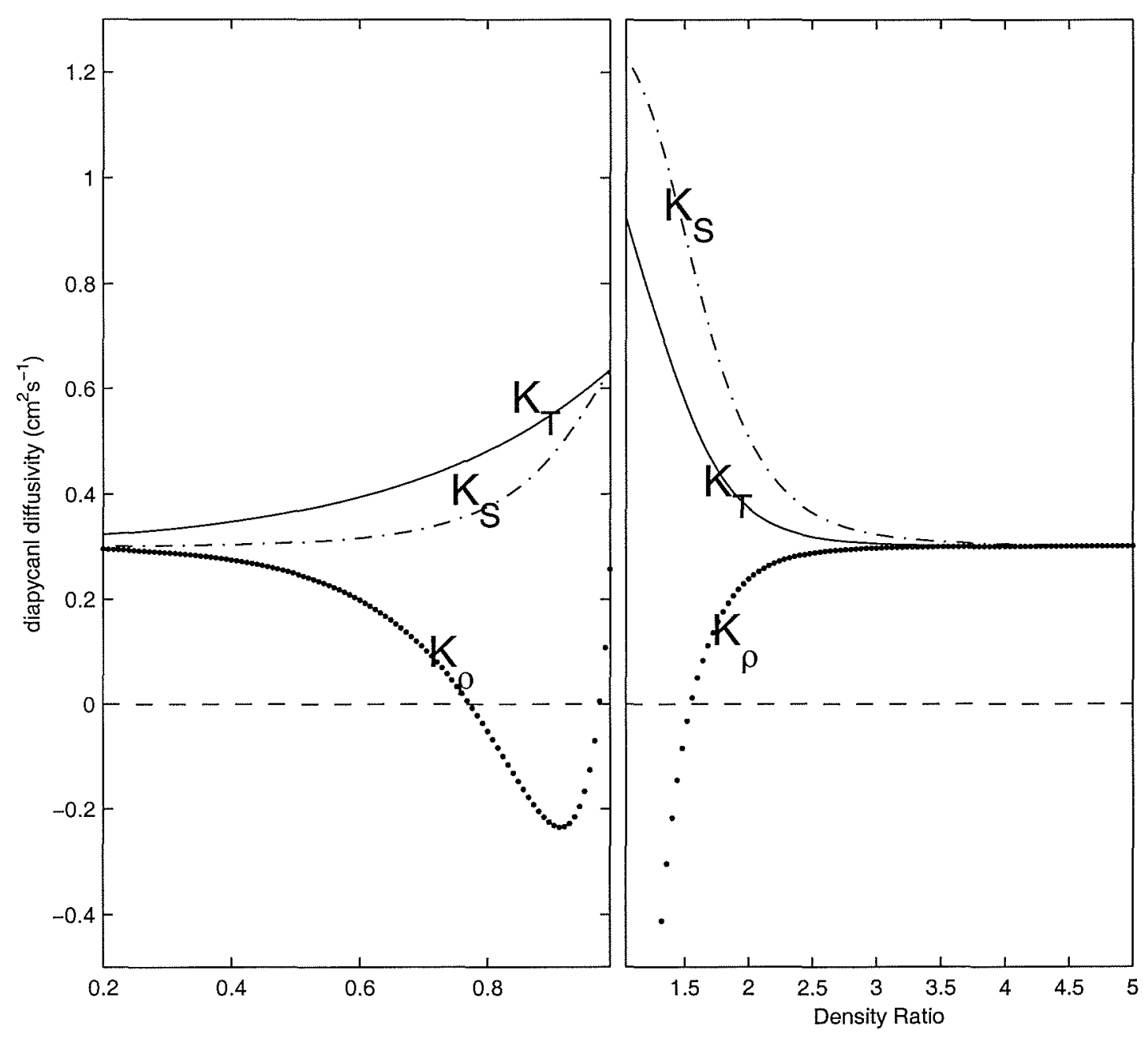

Figure 3.1: The change of diapycnal eddy diffusivities of temperature (solid line), salinity (broken line) and density (dotted line) with density ratio $R_{\rho}$. In the left half panel, where $R_{\rho}<1$, diffusive layering mixing occurs. In comparison, in the right half panel where $R_{\rho}>1$, salt fingers form. Note even though diffusive layering region and salt fingering region seem to be continuous at $R_{\rho}=1$, in the real ocean, normally there is a transition region between them where $R_{\rho}<0$. 


\subsubsection{Relaxation Boundary Conditions}

\section{Density ratio}

The density ratio is the key parameter in the study of double diffusion and here we give a simple argument to estimate its interior value based on the surface boundary conditions. In the present-day ocean, water tends to be warm and salty in the subtropical regions, and cold and fresh in the polar regions. Thus heat and salt oppose one another in terms of their effect on density. Due to these horizontal differences, the stratification forms as water is convected and sunk in the polar (equatorial) regions and then spreads at depth throughout the ocean for the thermal (haline) mode. Given the upper boundary conditions, a stratification favorable for double diffusion forms as a result. For example, in a thermal mode, cold fresh water sinks in the polar regions and spreads equatorward, and thus for most of the oceans, the warm and salty water overlies cold and fresh deep water; a condition favorable for fingering. In contrast, in the haline mode, warm salty water sinks in the equatorial regions and then spreads poleward, producing conditions favorable for diffusive layering. Therefore, the vertical density ratio can be approximated by the horizontal density ratio at the surface, which is essentially prescribed under relaxation boundary conditions:

$$
R_{\rho}=\frac{\alpha T_{z}}{\beta S_{z}} \sim \frac{\alpha \Delta T}{\beta \Delta S} \sim \frac{\alpha \Delta T^{*}}{\beta \Delta S^{*}}
$$

where $\Delta T, \Delta S$ are the north-south surface temperature and salinity differences, and $\Delta T^{*}, \Delta S^{*}$ are the corresponding differences in the prescribed values being relaxed to.

This definition of density ratio not only measures the relative contribution of heat and salt on the vertical density gradient, which in turn controls the strength of double diffusion, but also provides the relative contribution in determining the location for convection. When $R_{\rho}>1$, temperature dominates the density, deep water forms and sinks in the polar region, and we obtain a "thermal" mode of thermohaline circulation. This upper boundary condition provides a favorable condition for salt fingers. In contrast, when $R_{\rho}<1$, we obtain a "haline" mode and conditions favorable for diffusive layering. The above two processes will be discussed in turn below. 
Thermal Mode $\left(R_{\rho}>1\right)$

From the parameterization given in (3.1) - (3.2), we obtain the diagnostic diffusivity of density $K$ by using (3.16)

$$
K=K^{\infty}-\frac{0.3 K^{*}}{\left(R_{\rho}-1\right)\left[1+\left(R_{\rho} / R_{c}\right)^{n}\right]}
$$

Thus $K$ is always less than the background diffusivity $K^{\infty}$, with the deviation from $K^{\infty}$ depending on the local density ratio and other variables.

Using the $K^{2 / 3}$ power dependence of meridional overturning rate (MOC) on $K$ derived in Chapter 2 (2.18), we obtain:

$$
\frac{M_{S F}}{M_{C D D}}=\left[1-\frac{0.3 K^{*} / K^{\infty}}{\left(R_{\rho}-1\right)\left[1+\left(R_{\rho} / R_{c}\right)^{n}\right]}\right]^{2 / 3}
$$

where $M_{S F}$ is the MOC with salt fingers and $M_{C D D}$ is the MOC with conventional uniform vertical mixing $K_{T}=K_{S}=K^{\infty}$. From this relation we can see how sensitive the MOC is to the variables in the parameterization of salt fingers. This sensitivity is plotted in Figure 3.2.

To test the sensitivity results in the scaling analysis, numerical experiments are implemented in GFDL MOM2 and also plotted in Figure 3.2. The solid lines represent the analytical dependence derived in equation (3.20) and triangles are the individual numerical experiment results. The results are basically consistent. When we increase $K^{*}$, i.e, the contribution from double-diffusive mixing, the magnitude of the meridional overturning is reduced. Similarly when we increase $R_{c}$ or decrease $n$, allowing modest fingering to affect a greater portion of the water column, the thermohaline circulation weakens.

The relationship between $R_{\rho}$ and the eddy diffusivities for salt and heat is still not firmly established. Schmitt (1981) applied the laboratory-derived flux laws to observational data and estimated the eddy diffusivities for salt and heat. The maximum diffusivity due to salt fingers could reach $5.0 \mathrm{~cm}^{2} \mathrm{~s}^{-1}$ or more. However, given the uncertainty associated with the empirical flux laws and more understanding gained through the C-SALT (Schmitt et al., 1987) field program, we judged that a good first step was to use a somewhat conservative value for $K^{*}$, like $K^{*}=1.0 \mathrm{~cm}^{2} \mathrm{~s}^{-1}$. St. Laurent and Schmitt (1998) examined The North Atlantic Tracer Release Experiment 
(a)

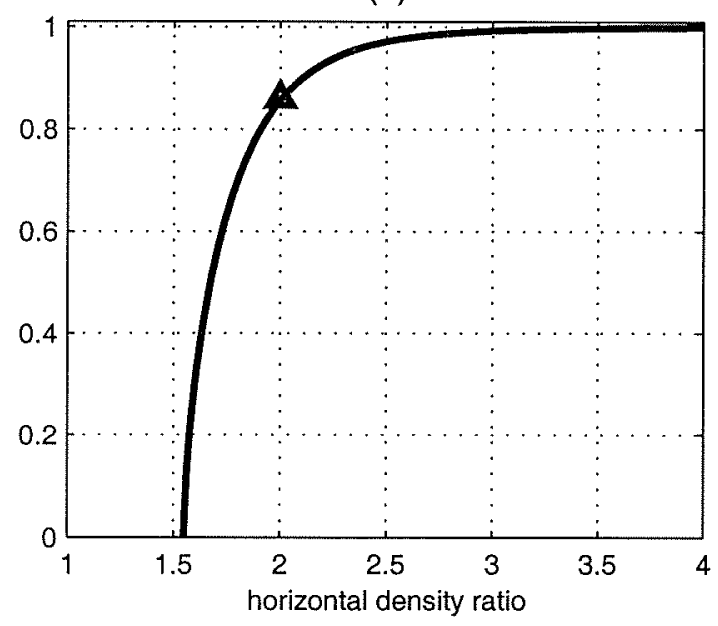

(c)

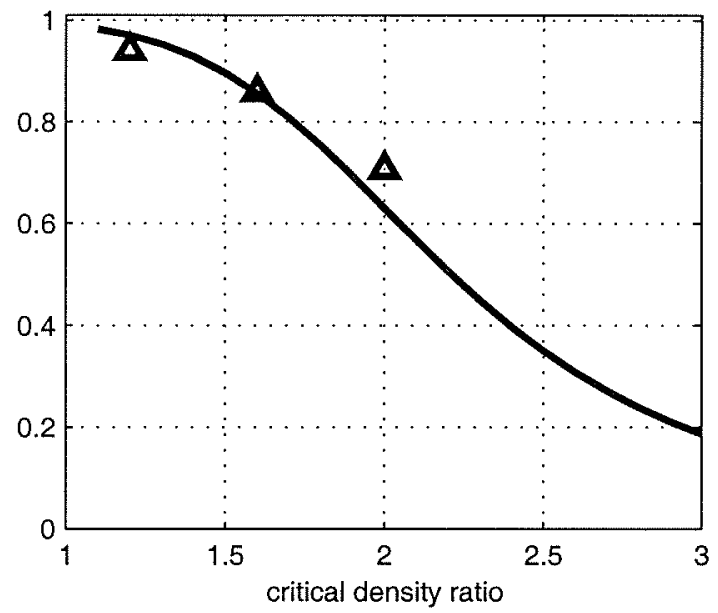

(b)

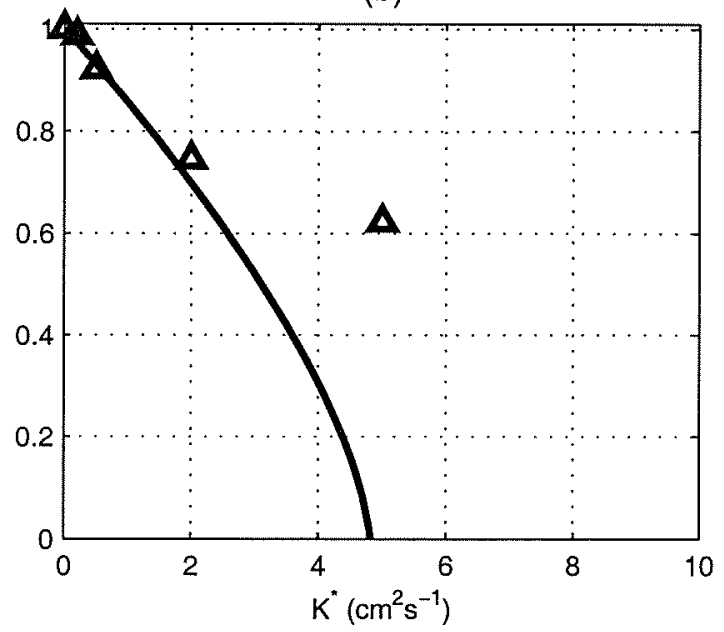

(d)

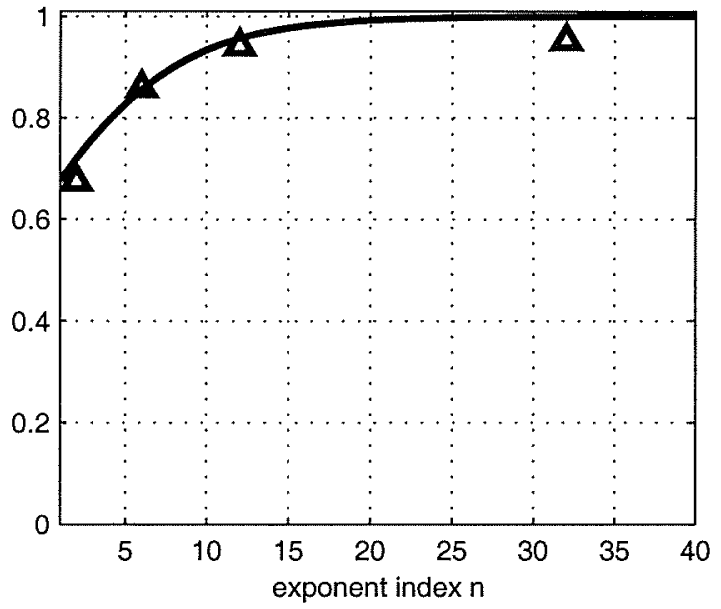

Figure 3.2: Sensitivity of $\frac{M_{S F}}{M_{C D D}}$ to the following variables in the parameterization of salt fingering (a) horizontal density ratio $R_{\rho}$, (b) the maximum diffusivity due to salt fingers $K^{*}$, (c) critical density ratio $R_{c}$ and (d) exponent index $n$. The solid line represents the results through scaling analysis and the triangles are the numerical experiment results. The variables are given as follows unless given as variable: $K^{*}=$ $1.0 \mathrm{~cm}^{2} \mathrm{~s}^{-1}, K^{\infty}=0.3 \mathrm{~cm}^{2} \mathrm{~s}^{-1}, R_{\rho}=2.0, R_{c}=1.6, n=6$. 
data, and found that $K_{T}=(0.08 \pm 0.01) \mathrm{cm}^{2} \mathrm{~s}^{-1}$ and $K_{S}=(0.13 \pm 0.01) \mathrm{cm}^{2} \mathrm{~s}^{-1}$ for the neutral surface at about $300 \mathrm{~m}$. Also they found that both $K_{T}, K_{S}$ increase with decreasing density ratio. As even a conservative value of $K^{*}$ leads to a marked difference in the thermohaline circulation, it suggests that more work should to be done to understand the role of salt fingers in oceanic mixing.

$R_{c}$ and $n$ can both affect the relation between $K_{T}, K_{S}$ and $R_{\rho}$. On the basis of the limited observational data, $K_{S}$ appears to be large for $R$ less than 1.5, falling sharply near $R_{\rho}=1.7$ and reaching background levels by $R_{\rho}=1.9$. Thus $R_{c}=$ 1.6, $n=6$ are reasonable based on observations of finestructure (Schmitt 1981) and microstructure (St. Laurent and Schmitt, 1998). Even though the theoretical condition for salt fingers is $1<R_{\rho}<100$, observational data suggests that significant fingering only occurs when $R_{\rho}$ is close to 1 . A similar conclusion holds for the diffusive layering. Therefore a relatively stronger vertical gradient of salt (temperature) is required for the salt fingering (diffusive layering) to be important in the diapycnal mixing processes.

\section{Haline Mode $\left(R_{\rho}<1\right)$}

For Kelley's parameterization, the diagnostic $K$ is:

$$
K=K^{\infty}-\frac{1-R_{F}}{1-R_{\rho}} C R_{\rho}^{1.9 / 3} e^{4.8 R_{\rho}^{0.72}}
$$

Then we can estimate its effects on MOC:

$$
\frac{M_{D L}}{M_{C D D}}=\left[1-\frac{1-R_{F}}{1-R_{\rho}} \frac{C R_{\rho}^{1.9 / 3} e^{4.8 R_{\rho}^{0.72}}}{K^{\infty}}\right]^{2 / 3}
$$

where $M_{D L}$ is the MOC with the implementation of diffusive layering.

There are two free variables in this parameterization: $R_{\rho}$ and $K^{\infty}$. The parameter sensitivity can be seen through Figure 3.3. It shows that when $R_{\rho} \rightarrow 0.9$, diffusive layering is strongest. In addition, the larger the background diffusivity is, the weaker the effects of diffusive layering on thermohaline circulation are. 
(a)

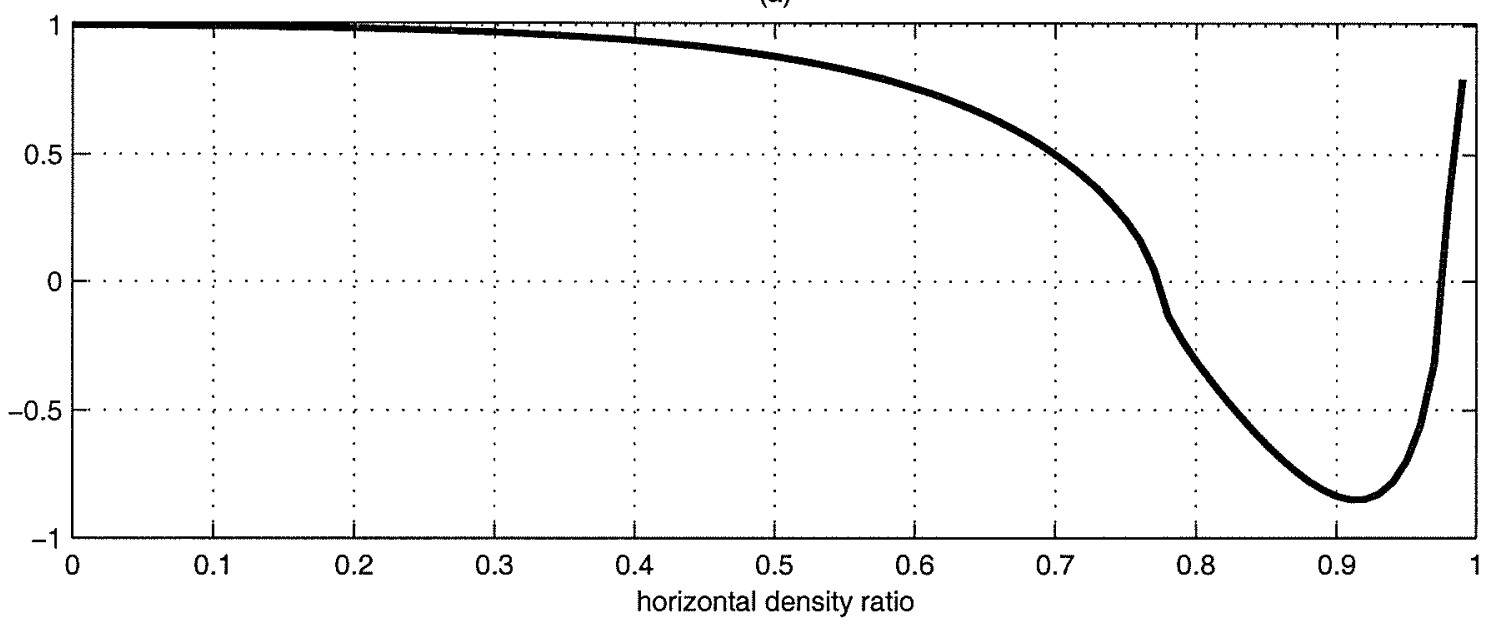

(b)

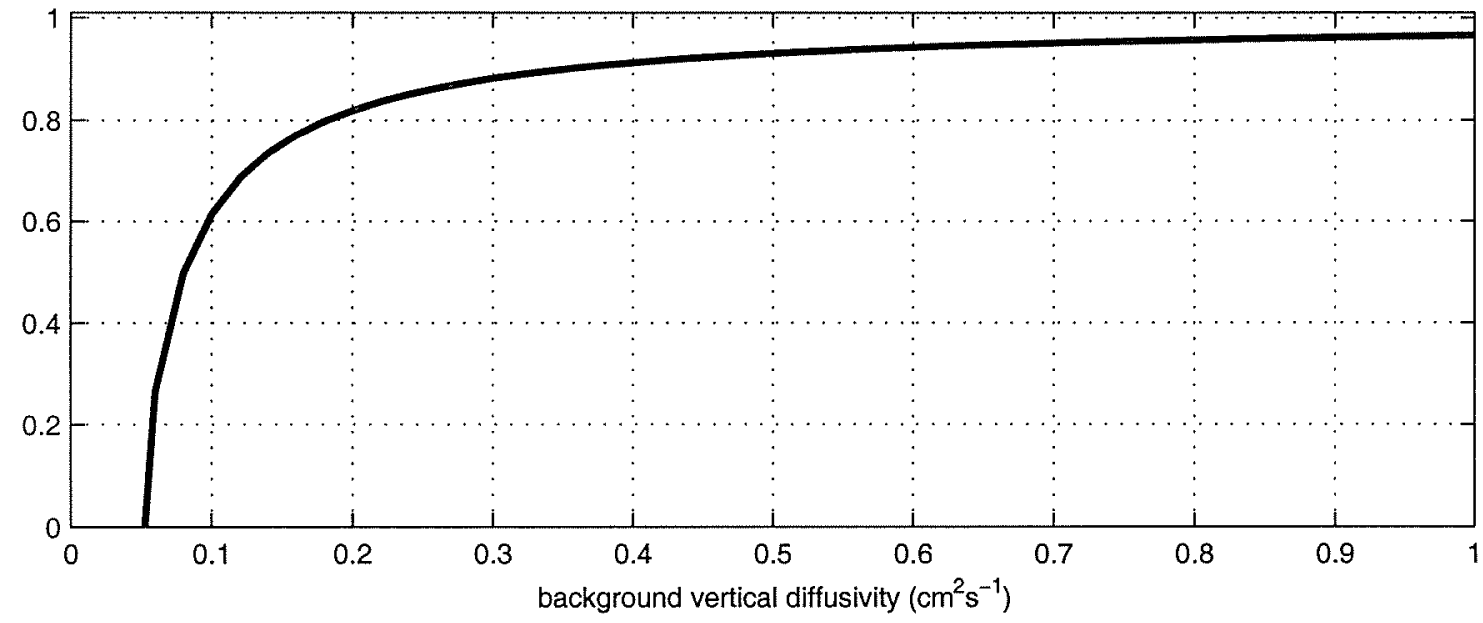

Figure 3.3: Sensitivity of ratio $\frac{M_{D L}}{M_{C D D}}$ to the following variables in the parameterization of diffusive laying: (a) the horizontal density ratio $R_{\rho}$ and (b) background vertical diffusivity $K^{\infty}$. 


\subsubsection{Flux Boundary Conditions}

\section{Density Ratio}

Under flux boundary conditions, the density ratio is defined in the fashion of (3.18), where the density ratio is also given by the horizontal ratio. In contrast to (3.18), the north-south temperature and salinity difference is given by (2.28) and (2.30) respectively, therefore we obtain

$$
R_{\rho}=\frac{\alpha T_{z}}{\beta S_{z}} \sim \frac{\alpha \Delta T}{\beta \Delta S}=\frac{\alpha Q}{\beta \bar{S} E \rho_{0} c_{p}}
$$

where $Q$ is the sea surface heat flux, $E$ is the sea surface freshwater flux, and $\bar{S}, \rho_{0}, c_{p}$ are the averaged salinity, density and specific heat of sea water respectively. Thus the density ratio measures the relative contribution of the heat and freshwater fluxes. Similar to the above section, when $R_{\rho}>1$, thermal forcing dominates and we obtain a "thermal" mode and the condition is salt fingering favorable; when $R_{\rho}<1$, we obtain a "haline" mode and conditions suitable for diffusive layering.

Thermal Mode $\left(R_{\rho}>1\right)$

The diagnostic diffusivity of density from parameterization (3.1) - (3.2) is obtained in (3.16). Then we can use the $K^{1 / 2}$ power law dependence of MOC on $\mathrm{K}$ in Chapter $2(2.33)$ to obtain

$$
\frac{M_{S F}}{M_{C D D}}=\left[1-\frac{0.3 K^{*} / K^{\infty}}{\left(R_{\rho}-1\right)\left[1+\left(R_{\rho} / R_{c}\right)^{n}\right]}\right]^{1 / 2}
$$

where all the variables are defined the same way as under relaxation boundary conditions.

\section{Haline Mode $\left(R_{\rho}<1\right)$}

Similarly, impacts of Kelley's parameterization of diffusive layering under flux boundary conditions can be expressed as

$$
\frac{M_{D L}}{M_{C D D}}=\left[1-\frac{1-R_{F}}{1-R_{\rho}} \frac{C R_{\rho}^{1.9 / 3} e^{4.8 R_{\rho}^{0.72}}}{K^{\infty}}\right]^{1 / 2}
$$


where $M_{D L}$ is the MOC with the implementation of diffusive layering.

The impact of double diffusion on thermohaline circulation under flux boundary conditions is very similar to that under relaxation boundary conditions, except for the difference in the power law dependence.

\subsubsection{Mixed Boundary Conditions}

\section{Density Ratio}

Similar to the density ratio defined in (3.18), under the mixed boundary conditions, we also assume that the density ratio is determined by the horizontal density ratio, which is given by the north-south temperature and salinity differences. However, in contrast to the relaxation boundary conditions, the density ratio is related to the strength of the meridional overturning and becomes part of the solution,

$$
R_{\rho}=\frac{\alpha T_{z}}{\beta S_{z}} \sim \frac{\alpha \Delta T}{\beta \Delta S}=\frac{\alpha \Delta T K}{\beta \bar{S} E D}
$$

Note that the density ratio defined here is the same as the $R$ in the scaling relation (2.44).

To differ with the conventional constant vertical diffusivity case, the free parameter in (2.44) defined by

$$
F=\frac{g K^{4}(\alpha \Delta T)^{4}}{f L^{2} E^{3}(\beta \bar{S})^{3}}
$$

now becomes a function of $R_{\rho}$, since $K=K\left(R_{\rho}\right)$ with the consideration of doublediffusive processes. To simplify the argument, we introduce a new parameter,

$$
F_{C D D}=\frac{g\left(K^{\infty}\right)^{4}(\alpha \Delta T)^{4}}{f L^{2} E^{3}(\beta \bar{S})^{3}}
$$

then the scaling relation (2.44) becomes

$$
R_{\rho}^{4}=F_{C D D}\left(K / K^{\infty}\right)^{4}\left|R_{\rho}-1\right|
$$

where $K$ is defined in (3.19) and (3.21) for the case of salt fingers and diffusive layers respectively. Since in the parameterization of double-diffusive mixing $K$ is strongly 
dependent on the density ratio $R_{\rho}$, the order of equation (3.29) becomes higher and thus no analytical solution can be obtained. To solve this equation, we have two options: (1) solve the equation numerically, thus the number of solutions should be equal to the order of equation, and then eliminate all unphysical solutions; (2) solve the above equation asymptotically, i.e., we start to solve the equation with $K=K^{\infty}$, and we will have three possible solutions. For the stable thermal mode, we use the parameterization of salt fingers and we'll have a diagnosed $K$, and then we use this diffusivity to solve the stable thermal solution of (3.29), and we repeat the above processes until the solution converges.

As discussed in Chapter 2, the unstable thermal mode is not observed in numerical models and there is special phenomenon "flushing" associated with the haline mode, thus here we only consider the effects of salt fingers on the stable thermal mode.

\section{Stable Thermal Mode $\left(R_{\rho}>1\right)$}

Here we solve (3.29) with $K$ given in (3.19). From Chapter 2, we know that the existence of the thermal mode depends on the relative contribution of diapycnal mixing and freshwater forcing, thus here we need to investigate not only how the magnitude of the stable thermal mode depends on the the parameterization of salt fingers, but also how the critical freshwater flux that determines the existence of the thermal mode is impacted by the above parameterization. Also, we wish to determine how the variables in (3.19) can change the above dependence.

The solutions are plotted in Figure 3.4. CDD represents the scaling results without considering double-diffusive effects, and other curves show the impact of different salt fingering parameterizations. The variable values for the salt fingers are the same as in (3.17) unless specified here. It is obvious that the salt fingering parameterization leads to a reduction in the critical freshwater flux required for the existence of the stable thermal mode. This is consistent with the analysis in Chapter 2, since the upper limit (critical value) of freshwater forcing depends on $K^{2 / 3}$. With the salt fingering parameterization, the vertical diffusivity of density is reduced and we expect a reduction in the critical freshwater forcing. From Figure 3.4, we also see the sensitivity to the variables in the parameterization of salt fingers: the critical freshwater 
(a)

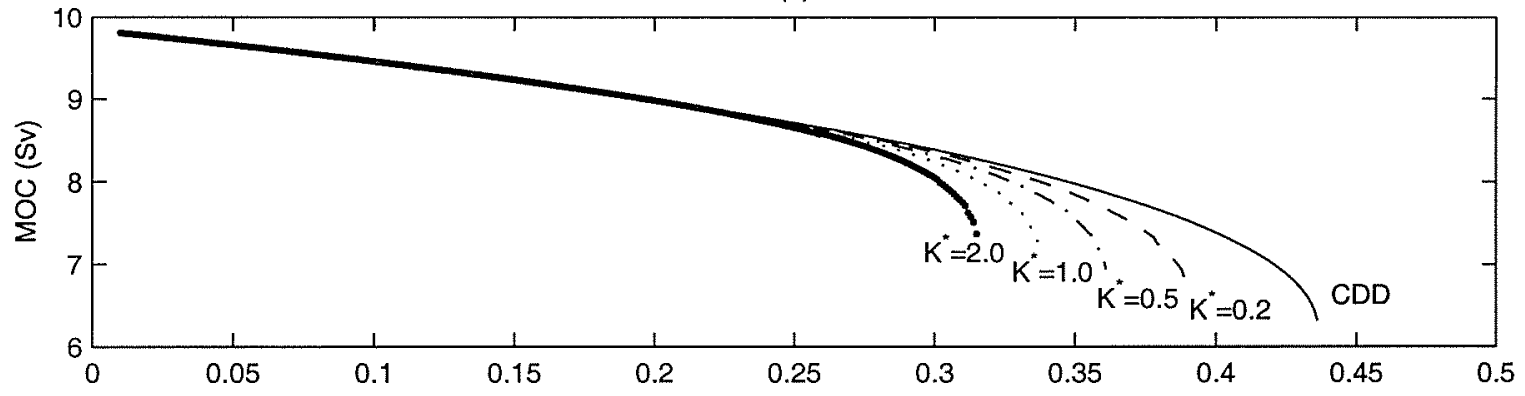

(b)

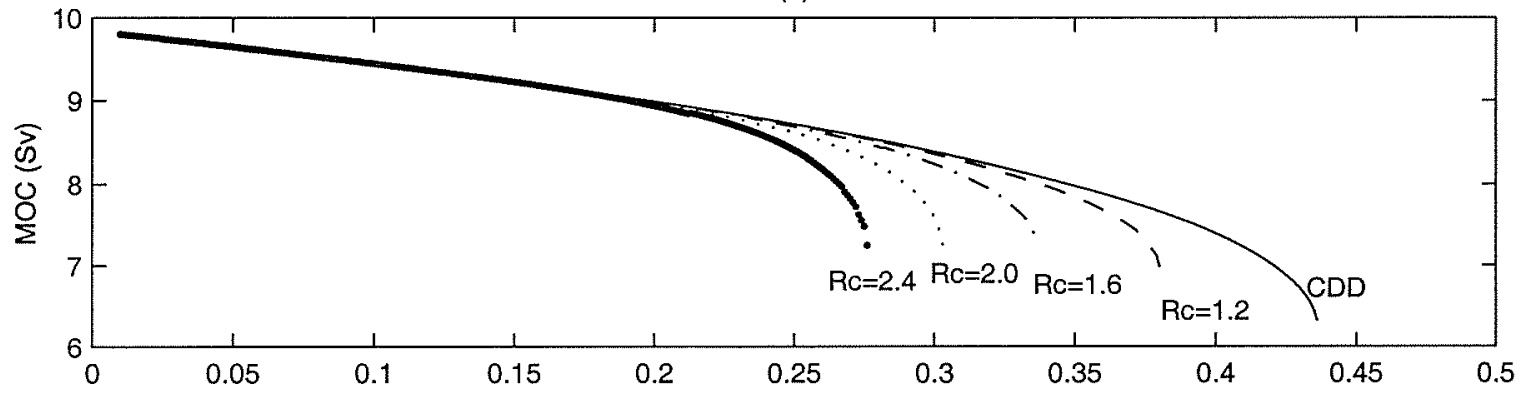

(c)

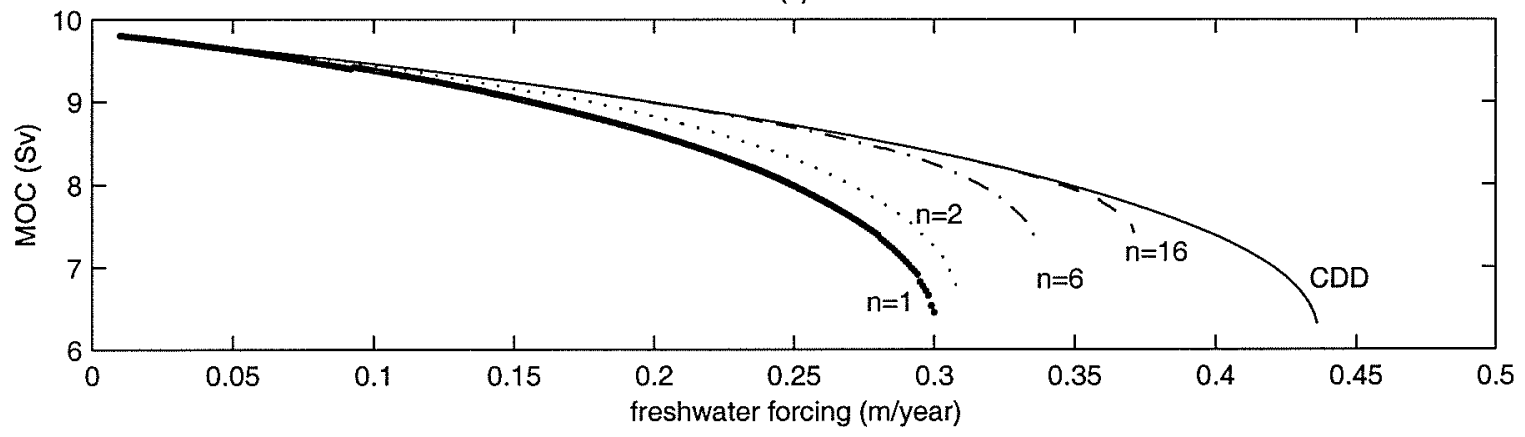

Figure 3.4: Sensitivity of the existence of the stable thermal mode on the freshwater forcing to the following variables in the parameterization of salt fingers, under mixed boundary conditions: (a)the maximum double-diffusive diffusivity $K^{*}$, (b) critical density ratio $R_{c}$ and (c) exponent index $n$. 
forcing also decreases with increased maximum diffusivity due to fingers $K^{*}$, larger critical density ratio $R_{c}$, or smaller exponent index $n$.

Another impact of salt fingering on the stability of the thermal mode is the dependence on the strength of freshwater forcing. When the freshwater forcing is relatively weak, the salt fingering hardly makes any difference compared with the CDD case. When the freshwater forcing is strong enough, the salt fingering parameterization starts to decrease the magnitude of the thermal mode, and the difference becomes larger with increasing freshwater forcing until the thermal mode breaks down when the critical freshwater forcing is reached. This is because the density ratio is part of the solution we are pursuing, and not a given external constant as it is under relaxation or flux boundary conditions. The magnitude of the density ratio decreases with increasing freshwater forcing. Given the strong dependence of the intensity of salt fingering upon density ratio, we speculate that for low freshwater forcing, since the density ratio is very high, salt fingering barely makes a contribution to the vertical mixing. As a result, it remains nearly identical with the CDD case.

\subsection{Summary}

The double-diffusive processes have been parameterized by diapycnal eddy diffusivities for heat and salt that differ and depend on the local density ratio, $R_{\rho}=\alpha T_{z} / \beta S_{z}$. A background diffusivity is applied to represent turbulent mixing in the stratified environment. The parameterization has three distinct features compared with the conventional constant eddy diffusivity assumption: (a) The vertical eddy diffusivities of temperature $\left(K_{T}\right)$ and salinity $\left(K_{S}\right)$ are different. For salt fingering mixing, $K_{S}>K_{T}$, and vice versa for diffusive layering mixing. (b) $K_{T}$ and $K_{S}$ both increase when local density ratio $R_{\rho}=\alpha T_{z} / \beta S_{z}$ is close to 1 , and there is a cut off in the $R_{\rho}$ dependence. (c) A constant diapycnal diffusivity applies for the non-doublediffusive turbulence mixing. When $R_{\rho}>3.0$ or $R_{\rho}<0.3$, the diapycnal diffusivity is dominated by turbulent mixing rather than double diffusion. (d) When the vertical temperature gradient becomes less than a critical value, no double diffusion is applied. This has the effect of eliminating double diffusion in the deep ocean, where 
there is little evidence for its importance. The implementation of this double-diffusive parameterization leads to a decrease of the downward buoyancy diffusion.

By assuming that the internal density ratio is determined by the horizontal density ratio, the impacts of double-diffusive processes on the thermohaline circulation can be estimated by scaling analysis. Under relaxation and flux boundary conditions, the magnitude of the meridional overturning rate is reduced, with the extent of reduction being sensitive to the variables in the parameterization. Under mixed boundary conditions, the critical freshwater forcing necessary for the existence of the thermal mode is reduced by the introduction of double diffusion. 


\section{Chapter 4}

\section{Impacts of Double-diffusive}

\section{Processes on Thermohaline}

\section{Circulation under Restoring}

\section{Boundary Conditions}

\subsection{Introduction}

The thermohaline circulation of the ocean plays an important role in Earth's climate system, especially in being responsible for most of the oceanic part of the poleward heat transport. The large-scale structure of the thermohaline circulation and its variability has been studied extensively in models (Weaver and Hughes, 1992; Huang, 1995). One result of these studies is the finding that the strength of the meridional overturning cell (MOC) is strongly dependent on the value of the vertical (or diapycnal) eddy diffusivity $(\mathrm{K})$, which is the mechanism that provides the necessary warming of the rising abyssal waters. Bryan (1987) found an approximate $K^{1 / 3}$ dependence of the MOC; however, his model runs may not have reached equilibrium; later work has found a $K^{2 / 3}$ dependence (Marotzke, 1997; Zhang et al. 1998b), when 
the conventional "relaxation" boundary conditions are applied for the surface forcing. Whatever the specific power law dependence, the continual production of cold deep water at high latitudes requires vertical mixing to close the circulation (Munk, 1966).

Observational microstructure work in recent years (Gregg, 1989; Polzin et al, 1995) has indicated that turbulent mixing in the ocean interior is generally much weaker than inferred from the large scale budget approach of Munk (1966), Hogg et al. (1982) and others. However, evidence is emerging that turbulence near rough topography may be sufficiently enhanced to provide the required downward heat flux (Toole et al., 1994; Polzin et al., 1996; Polzin et al., 1997). One problem with near-bottom mixing is that the stratification is weak in the abyss, so heat fluxes tend to be small, even with very large eddy diffusivities. This reduced effectiveness of deep internal wave induced turbulence (the mixing of already mixed water) leads us to examine the other mixing processes occurring in the more strongly stratified thermocline.

The primary processes that must be considered are the double-diffusive instabilities of salt fingering, which occurs when temperature and salinity both decrease with depth, and diffusive layering, which occurs when temperature and salinity both increase with depth. It is well known that on the molecular level heat and salt have diffusivities which are two orders of magnitude different. This difference drives convective motions even if the overall density profile is stable. Substantial evidence is now available that these processes play a significant role in ocean mixing (Schmitt, 1994). Their principal effect is to transport heat and salt at different rates in the vertical, and cause a net upgradient flux of buoyancy. A number of recent modeling studies show that such differential transports have important effects on the stability and structure of the large-scale thermohaline circulation.

In particular, Gargett and Holloway (1992, hereafter GH92) first investigated the effects of a simple double-diffusive parameterization in an ocean model. They showed that the steady-state characteristics of low resolution GCMs used in climate studies are very sensitive to the ratio of the vertical eddy diffusivities for salinity and temperature. Large differences in meridional transports resulted due to the upgradient buoyancy flux which forced different advective-diffusive balances to be realized.

Given the simplicity in the GH92 parameterization, we proposed a more accurate parameterization for double-diffusive processes in Chapter 3. This is applied in the 
climate-scale numerical model runs described below. However, the parameterization of mixing processes, both vertical and horizontal, requires careful treatment in numerical models. The oceanic circulation involves extremely broad scales in both time and space; thus, we can never resolve all the temporal and spatial scales in numerical models. Consequently, for the foreseeable future, sub-grid-scale phenomena must be parameterized in Ocean General Circulation Models (OGCM). In fact, determining suitable parameterizations of sub-grid-scale phenomena is one of the most critical problems in numerical modeling of the general circulation. Early ocean circulation models were mostly based on z-coordinates, and sub-grid-scale mixing was parameterized in terms of constant horizontal and vertical mixing. However, such simple horizontal/vertical mixing schemes can introduce strong artificial cross-isopycnal mixing near fronts, such as the Gulf Stream. To overcome the artificial cross-isopycnal mixing in low-resolution z-coordinates models, a numerical technique of rotating the mixing tensor has been proposed by Redi (1982), and it has been implemented and tested here.

In addition, Gent and McWilliams (1990) pointed out that there is an extra advection term in the tracer balance equation of non-eddy resolving models, whose existence is due to the Lagrangian mean transport of the meso-scale eddies. They introduced a parameterization based on a downgradient diffusion of the isopycnal layer thickness in adiabatic flow. The addition of this transport term makes the tracer conservation equations in a non-eddy resolving model self-consistent, and eliminates the unphysical background horizontal diffusion required in the earlier version of the isopycnal/diapycnal mixing schemes. The dynamic effect and model sensitivity on the isopycnal/diapycnal mixing has been discussed in several recent papers (Gent et al., 1995; Danabasoglu and McWilliams, 1995).

In this study we will focus on the parameterization of diapycnal mixing in connection with double-diffusive processes. Based on results from theory, laboratory experiments, and oceanic observations, the intensity of double diffusion must depend on the local density ratio $R_{\rho}=\alpha T_{z} / \beta S_{z}$. There is strong evidence indicating that double diffusion is important in controlling the diapycnal mixing process in the ocean only if $R_{\rho}$ is sufficiently close to one. Thus, we proposed a conservative parameterization of double diffusion that reflects this dependence (Chapter 3). This parameterization is applied to the GFDL MOM2 (Pacanowski, 1995) model. The controlling experiments and 
results are presented and interpreted in Section 4.2. In addition, sensitivity study experiments are presented in Section 4.3 in order to understand the response of the numerical model to the proposed double diffusive parameterization, and a summary follows in Section 4.4.

\subsection{Control Experiments}

\subsubsection{The Numerical Experiments}

The GFDL MOM2 is used in these experiments. The annual zonally averaged climatological surface wind stress (Hellerman and Rosenstein, 1983), SST and SSS (Levitus, 1982) are also used in these experiments. The first layer temperature and salinity are relaxed to the SST and SSS climatology, with a relaxation time of 30 days.

The horizontal and vertical momentum viscosity coefficients are $A_{h}=1.0 \times 10^{10} \mathrm{~cm}^{2} \mathrm{~s}^{-1}$ and $A_{v}=20 \mathrm{~cm}^{2} \mathrm{~s}^{-1}$ respectively. For the tracer equations, the eddy transport parameterization of Gent and McWilliams (1990) and isopycnal/diapycnal mixing is used, and no background horizontal diffusion is needed. We take $K_{I S O}=K_{I T D}=$ $5 \times 10^{6} \mathrm{~cm}^{2} \mathrm{~s}^{-1}$, where $K_{I S O}$ is the isopycnal diffusion coefficient and $K_{I T D}$ is the downgradient diffusivity of the isopycnal thickness.

Three control experiments will be discussed here, the first with the Double-Diffusive Parameterization (DDP hereafter) for temperature and salinity, and the second with the diffusivity given in a similar fashion to GH92 (Gargett and Holloway Diffusivity, GHD hereafter), i.e., the diffusivities for heat and salt are different but both kept constant everywhere, and the third with the conventional assumption of $K_{S}=$ $K_{T}=$ const, (Constant Diapycnal Diffusivity, CDD hereafter). Gargett and Holloway (1992) discussed many experiments in their paper, but given the differences in the formulation of the numerical model, particularly the inclusion of GM90, we deemed it worthwhile to run an experiment similar to theirs and to compare the results directly.

The variables in the double-diffusion parameterization are defined in (3.17). Note that this parameterization is more conservative than that of Schmitt (1981). This is adopted because results of the C-SALT observations (Schmitt, 1988) indicate that a 
modest reduction in mixing coefficient was appropriate. The uncertainties associated with these variables and vertical resolution will be discussed in details in the next section.

We designed these three experiments in such a way that everything is identical, except for the parameterization of diapycnal diffusion. All the experiments are run from the same initial conditions and under the same boundary conditions for 8,000 years. For the reason of comparability, we calculate the basin-averaged $K_{T}$ and $K_{S}$ in run DDP: $\bar{K}_{T}=0.326 \mathrm{~cm}^{2} \mathrm{~s}^{-1}$ and $\bar{K}_{S}=0.366 \mathrm{~cm}^{2} \mathrm{~s}^{-1}$, and then we use $K_{T}=0.326 \mathrm{~cm}^{2} \mathrm{~s}^{-1}$ and $K_{S}=0.366 \mathrm{~cm}^{2} \mathrm{~s}^{-1}$ in run GHD and $K_{T}=K_{S}=0.346 \mathrm{~cm}^{2} \mathrm{~s}^{-1}$ in run CDD, therefore the average diffusivities of both scalars are equal in all three experiments.

A technical issue is the computational cost of adding on the double-diffusive parameterization. For a 100-year integration on an SGI workstation, the CPU time is 1256, 1120 and $1113 \mathrm{CPU}$ seconds for run DDP, GHD and CDD respectively. Thus, the double-diffusive parameterization takes approximately $10 \%$ extra computational time, a rather modest cost.

\subsubsection{Diffusivity Variability in Run DDP}

The major difference between DDP and GHD/CDD is that $K_{T}$ and $K_{S}$ in run DDP depend on the spatial variability of density ratio, $R_{\rho}$. It is found that salt fingers are the dominant double-diffusive phenomenon, thus here we only map out the distribution of the salt diffusivity and the corresponding $K_{T}$ distribution can be deduced accordingly.

To visualize the complicated three-dimensional structure of the circulation we plot several two-dimensional maps of $K_{S}$ in Figure 4.1.

There are two distinct features in the $K_{S}$ distribution:

(1) The double diffusion is confined within the upper 1500 meters, thus only the upper 2000 meters are plotted in Figure 4.1 (c)-(f). Double diffusion is stronger near the bottom of the thermocline (e.g., layer 7 in Figure 4.1 (b)) than in shallower layers (e.g. layer 4 in Figure 4.1 (a)). Weak double-diffusive activity in the deep part of the model ocean is due to the additional constraint (3.3), which essentially 
(a)

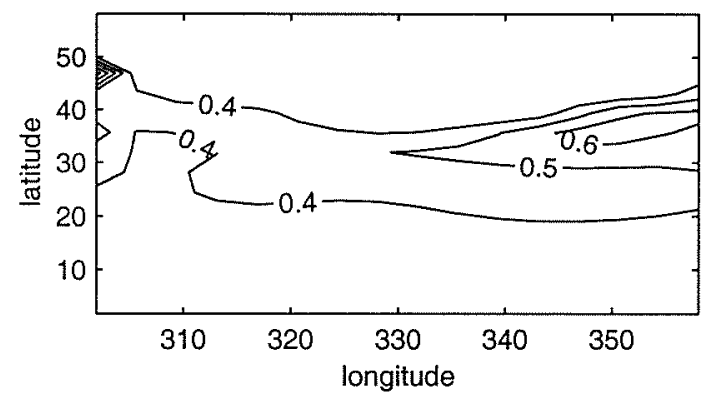

(c)

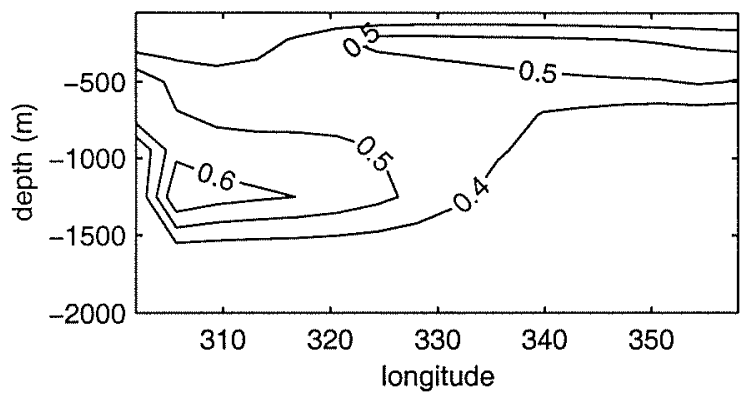

(e)

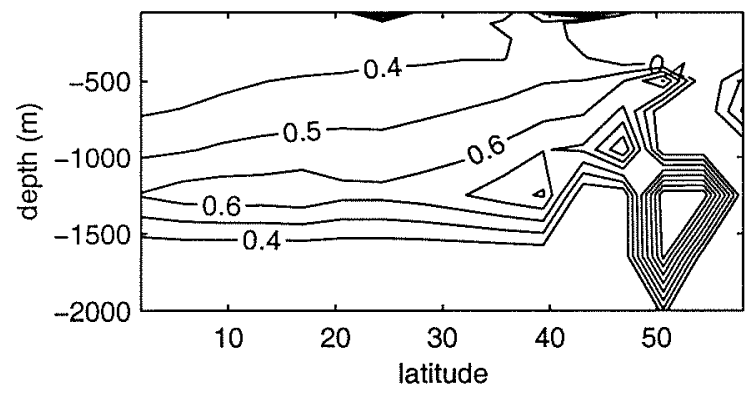

(b)

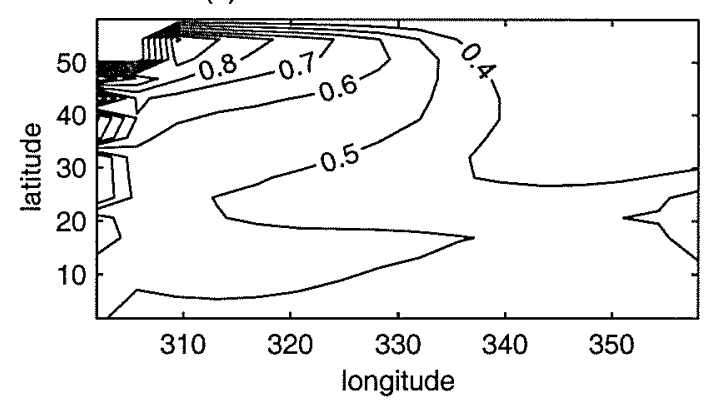

(d)

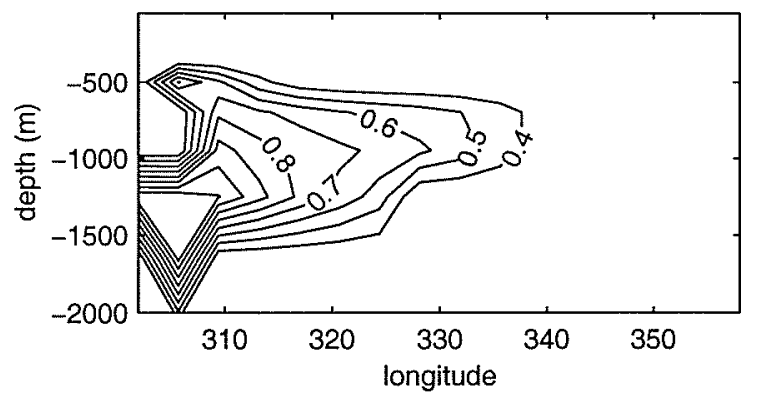

(f)

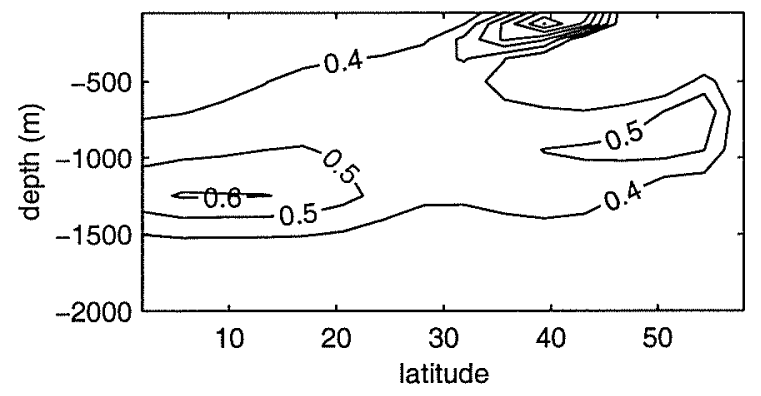

Figure 4.1: The $K_{S}$ distribution in run DDP of different sections: (a) horizontal section in the upper thermocline $(\mathrm{k}=4)$; (b) horizontal section in the lower thermocline $(\mathrm{k}=7)$; (c) zonal section in the subtropical gyre $(\mathrm{j}=10)$; (d) zonal section in the subpolar gyre $(j=15)$; (e) meridional section close to Western Boundary $(i=3)$; ( $f$ ) meridional section in the interior $(\mathrm{i}=10)$. The contour interval is $0.1 \mathrm{~cm}^{2} \mathrm{~s}^{-1}$. 
limit the double-diffusive processes in the deep ocean where the vertical gradients in the numerical model are weak. This constraint also reflects the fact that we have no reliable observational data to support double-diffusive phenomena in the deep ocean. Such a constraint can be modified in the future based on new observations.

(2) $K_{S}$ is stronger in the western boundary region than in the interior, indicating strong salt fingering there. The strong salt fingering is associated with the strong vertical temperature and salinity gradients within the western boundary region. According to the classic picture of the oceanic general circulation there are surface and deep western boundary currents. The strong surface western boundary current brings warm and salty water northward, and the deep western boundary current brings cold and fresh water southward along the western boundary. As a result, the temperature and salinity distribution within the western boundary region is dominated by horizontal advection, which maintains strong vertical gradients of temperature and salinity along the path of the western boundary currents, and this is the region most favorable for salt fingering. In comparison, vertical diffusion may play a more prominent role in the oceanic interior, and thus limits the vertical gradient of temperature and salinity there. As a result, salt fingering in the ocean interior is not as strong as within the western boundary region.

\subsubsection{Meridional Overturning}

The thermohaline circulation can be visualized by plotting the zonally integrated meridional overturning streamfunction. The structure of the thermohaline circulation in the three cases (Figure 4.2) is similar, but the magnitudes are significantly different. The maximum overturning rate in run CDD is $6.17 \mathrm{~Sv}\left(1 \mathrm{~Sv}=1.0 \times 10^{6} \mathrm{~m}^{3} \mathrm{~s}^{-1}\right)$; while it is only $4.79 S v$ in run DDP, a $22 \%$ decrease compared with run CDD. In run GHD the meridional overturning is $5.55 S v$, a $10 \%$ decrease compared with run CDD. There are traces of two-grid noise near the equator in all the three experiments, which may be due to the coarse vertical resolution (Weaver and Sarachik, 1990). In addition, a very weak reversed meridional cell in the equatorial deep ocean appears in run GHD and CDD. However, there is no reversed cell in DDP, and this may be due to the fact that the double diffusion parameterization helps to eliminate the local water column instability which leads to the local reversed cell. 
(a)

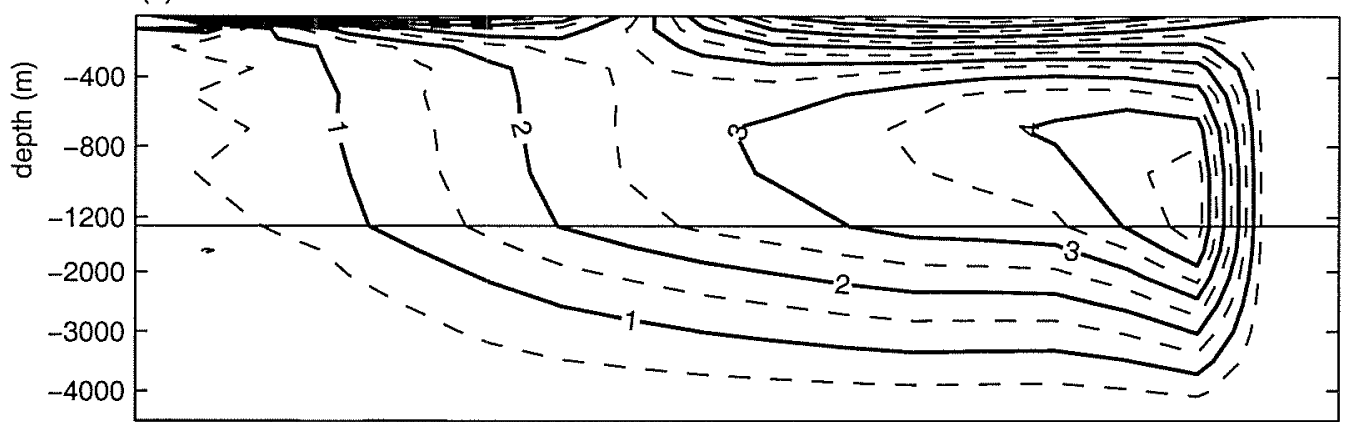

(b)

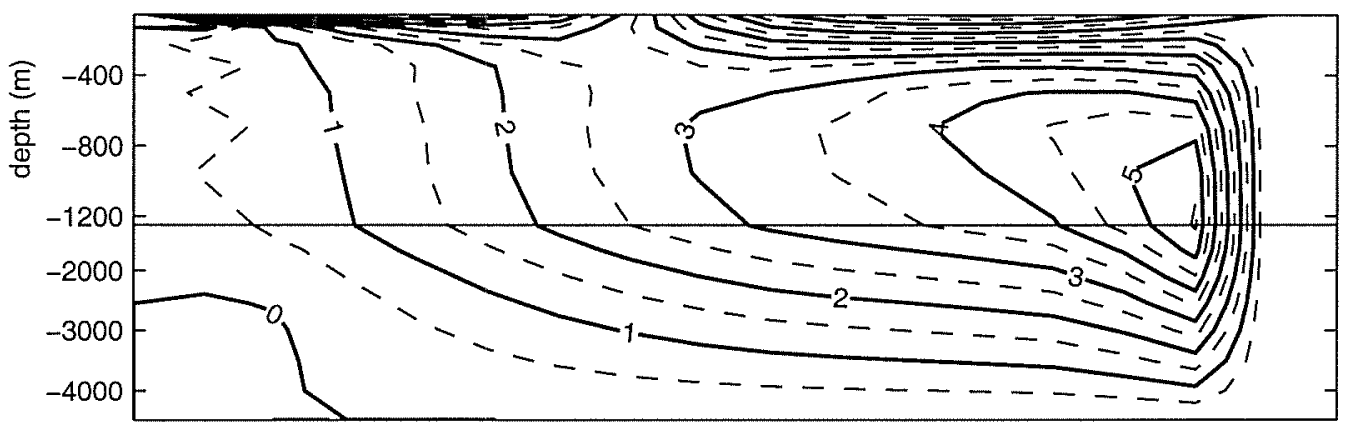

(c)

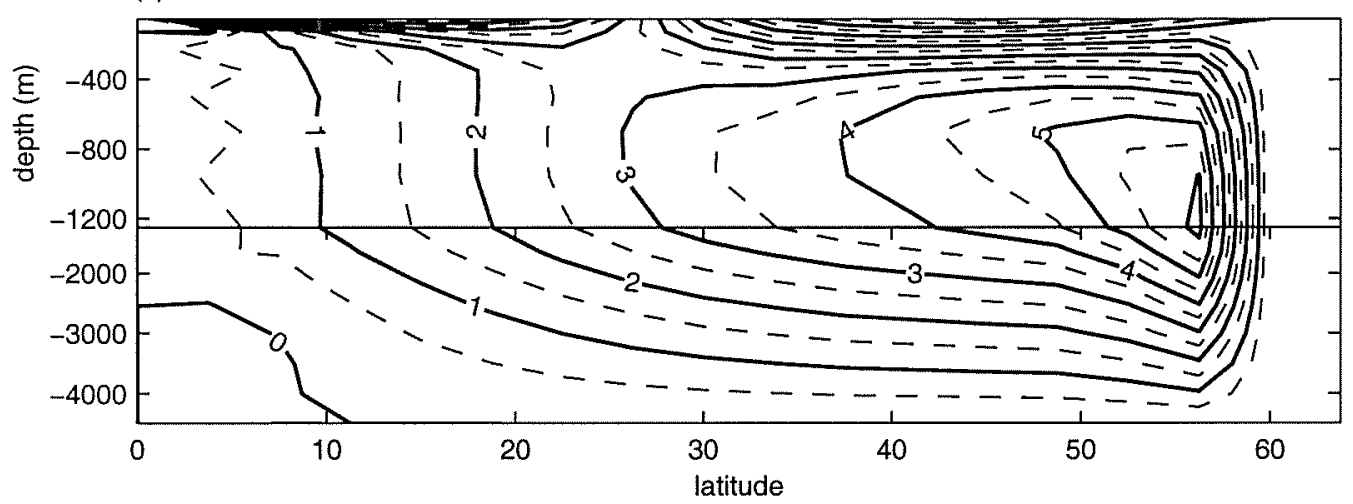

Figure 4.2: The zonally integrated meridional overturning volume streamfunctions for run (a) DDP (b) GHD and (c) CDD. Contour interval is $0.5 S v(1 S v=1.0 \times$ $\left.10^{6} \mathrm{~m}^{3} \mathrm{~s}^{-1}\right)$. 
According to the classical Stommel-Arons theory (Stommel and Arons, 1960), deep water moves equatorward in a western boundary current after its formation at high latitudes. The deep water returns to the source region through basin-wide uniform upwelling in the ocean interior. To reach an equilibrium, diapycnal advection (upwelling) must be compensated by diapycnal diffusion. Thus, the magnitude of the thermohaline circulation is very sensitive to the diapycnal diffusivity of density, as shown by Bryan (1987).

Given the importance of the diapycnal diffusivity of density, we plot the relation of $K_{\rho}$ and $R_{\rho}$ in Figure 4.3. In run CDD, $K_{\rho}=K_{S}=K_{T}$ is constant; however, in run GHD and DDP, $K_{\rho}$ depends strongly on $R_{\rho}$. In the region of diffusive layering $\left(R_{\rho}<1\right), K_{\rho}$ in run GHD is larger than the constant $K_{T}$ and $K_{S}$ specified in the experiment. This is contrary to the common belief that diffusive layering reduces or even reverses the vertical buoyancy diffusion, as in run DDP. In GH92, this drawback was avoided by giving $d=K_{S} / K_{T}>1$ or $d=K_{S} / K_{T}<1$ according to whether salt fingering or diffusive layering is favorable; thus, in both regimes $K_{\rho}$ is either reduced or with the sign reversed, in accordance with double-diffusive processes.

In the experiments studied here, salt fingering is the dominant double-diffusive process; therefore, only a very small region in run GHD is affected by the unphysical representation of diffusive layering process. The circulation is primarily controlled by salt fingering, and the relation between the density ratio and density diffusivity is plotted in the right half of Figure 4.3. We can see that both GHD and DDP are consistent with the salt fingering mechanism while displaying significant quantitative differences. First of all, given the same $R_{\rho}, K_{\rho}$ is biggest in run CDD, and then GHD, and smallest in run DDP. This difference becomes larger when $R_{\rho}$ is closer to 1 . As we will discuss later, the implementation of a double-diffusive parameterization has an effect on the distribution of density ratio (Turner Angle), which leads to a wider area of double-diffusively favorable conditions. Thus we can imagine that the difference between the model runs may be greater than the diffusivity differences at the same $R_{\rho}$.

Since downward buoyancy diffusion in run DDP is weaker than in run GHD and CDD, a smaller amount of upwelling is needed to balance the weaker density diffusion. Thus, the magnitude of the thermohaline circulation in run DDP is reduced. Furthermore, upwelling is not spatially uniform. In fact, upwelling within the western 


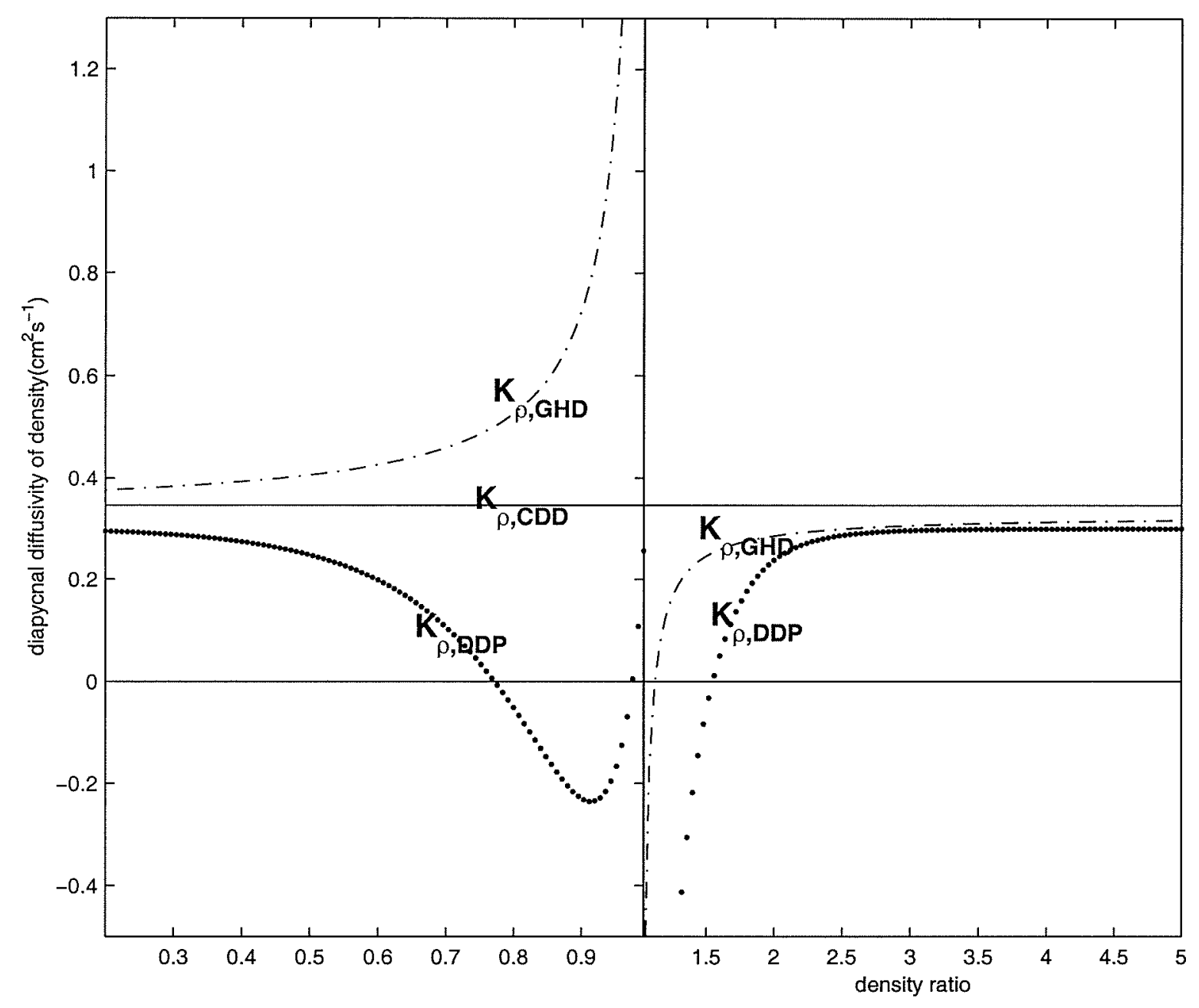

Figure 4.3: Relation between $K_{\rho}$ and $R_{\rho}$ in three different experiments. 
boundary region is two orders of magnitude stronger than in the interior in all the runs (Huang and Yang, 1996); thus, upwelling within the western boundary region constitutes an essential part of the upward limb of the thermohaline circulation. As discussed in section 2 , when double diffusion is sufficiently strong ( $R_{\rho}$ close to 1 ), buoyancy diffusion is reduced or reversed. Strong double diffusion in the western boundary region (Figure 4.1) dramatically reduces the downward buoyancy diffusion and can alter the direction of buoyancy diffusion (make it upward) locally. Even though diapycnal diffusion in the ocean interior is less affected, the total amount of the diapycnal diffusive flux of buoyancy is reduced and therefore the thermohaline circulation becomes weaker.

\subsubsection{Poleward Heat Transport}

With significant changes in the magnitude of the thermohaline circulation, it is not surprising that the poleward heat transport is also affected by the different parameterizations of diapycnal processes (Figure 4.4). The poleward heat transport at all latitudes is weakest in run DDP and strongest in run CDD, while it is intermediate in run GHD. The difference in poleward heat flux is most significant at mid-latitudes. The maximum poleward heat flux in run CDD is $0.255 \mathrm{PW}$, and it is $0.246 \mathrm{PW}$ in run GHD and $0.236 \mathrm{PW}$ in run DDP, with a $4 \%$ and $8 \%$ decrease respectively. Note that the decrease in the heat transport is less than the decrease in the thermohaline circulation. This can be explained as follows. First, the poleward heat transport is proportional to the meridional overturning rate multiplied by the temperature difference between the surface and deep water, and the maximum poleward heat transport is found in mid-latitudes, where the percentage of reduction of the thermohaline circulation at that latitude is less than that of the maximum overturning rate, and the temperature difference remains nearly the same for two experiments. Second, the wind driven gyre and Ekman cell also contribute to the poleward heat transport, which are controlled by the surface wind stress and remain almost the same for both experiments. 


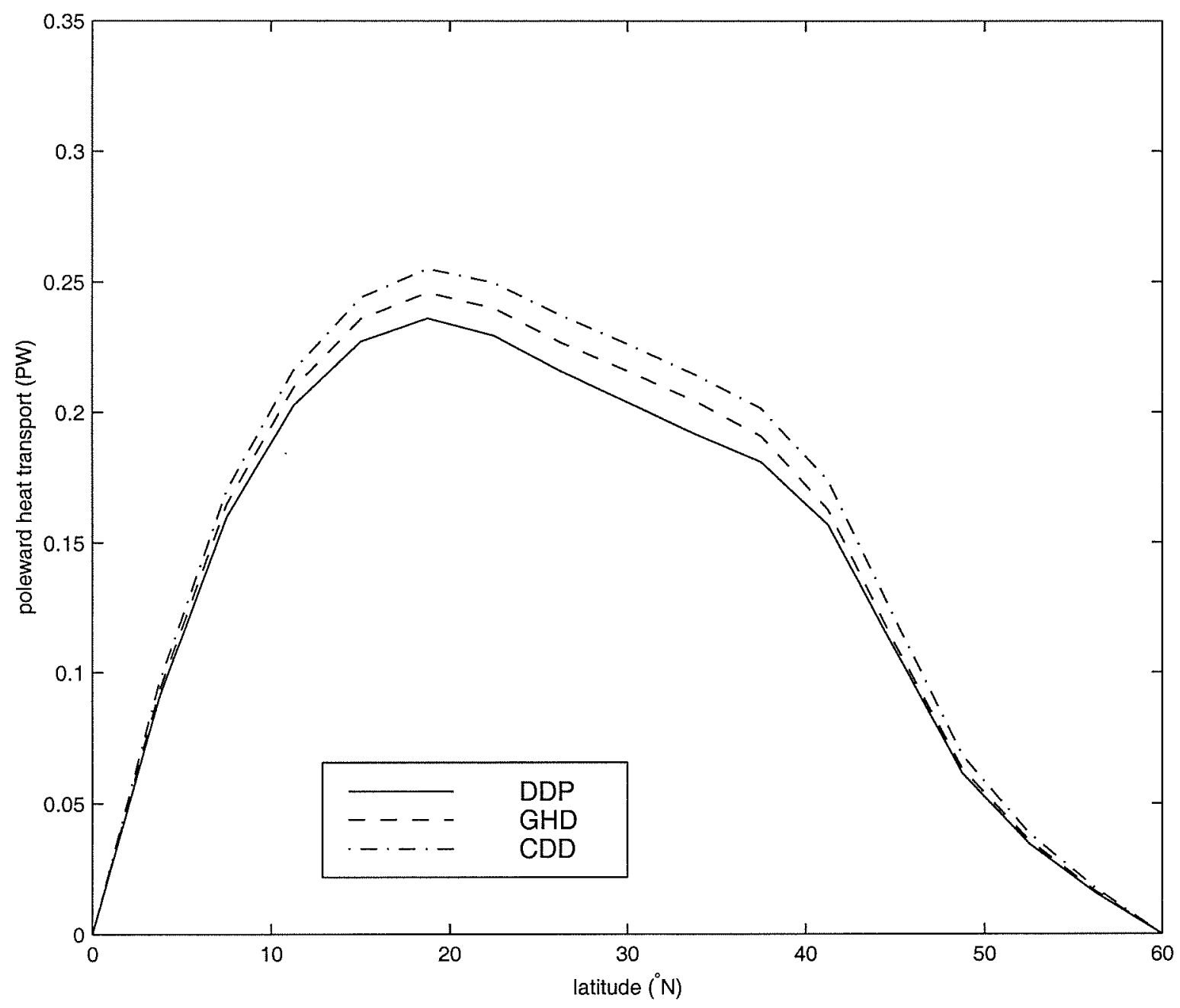

Figure 4.4: The poleward heat transport for the three experiments. 


\subsubsection{Turner Angle}

Since $R_{\rho}$ can vary from $-\infty$ to $\infty$ and it cannot represent the convective overturning regime, we describe the spatial structure of the density ratio by mapping the Turner angle $T u$. Ruddick (1983 and personal communication) defined the Turner angle as the four quadrant arctangent of $R_{\rho}$, i.e.,

$$
T u=\arctan \left(\frac{R_{\rho}+1}{R_{\rho}-1}\right)
$$

Thus salt fingering occurs for $45^{\circ}<T u<90^{\circ}$, and it is strongest when $T u$ is close to $90^{\circ}$. In comparison, diffusive layering occurs for $-90^{\circ}<T u<-45^{\circ}$ and when $T u$ is close to $-90^{\circ}$, diffusive layering is strong. When $-45^{\circ}<T u<45^{\circ}$, the fluid is in the non-double-diffusive regime. Other $T u$ values are within the gravitationally unstable region.

The Turner angle maps from the three experiments are complex. $T u$ at level $7(950 \mathrm{~m})$ and level $13(3500 \mathrm{~m})$ is plotted in Figure 4.5. Differences among three experiments are obvious: (a) Near the bottom of the thermocline (level 7), salt fingering favorable conditions are found in all experiments. Interestingly, the $T u$ in run DDP is lowest, and is highest in run CDD, indicating that salt fingering mixing in DDP is reinforced, especially near the western boundary current (WBC). This also occurs in run GHD, though not as strong as in run DDP. (b) In the deep ocean (level 13), the Turner angle in run CDD is only marginally critical in limited regions, but salt fingering (in the subtropical gyre) and diffusive layering (in subpolar gyre) both occur in run DDP. In run GHD, however, only the salt fingering favorable condition is found, and it is weaker than run DDP.

The introduction of double diffusion appears to change the distribution of the Turner angle. In these model runs (GHD,DDP), this parameterization renders the water column even more favorable to double diffusion. Although both salt fingering and diffusive layering are possible in run DDP, strong salt fingering appears over most of the basin, while diffusive layering is confined to narrow regions in the subpolar gyre near the surface or close to the bottom. The simple representation of double diffusion in GH92 makes the deep water more salt fingering favorable but eliminates the regime for diffusive layering as in run DDP and CDD. 
(a)

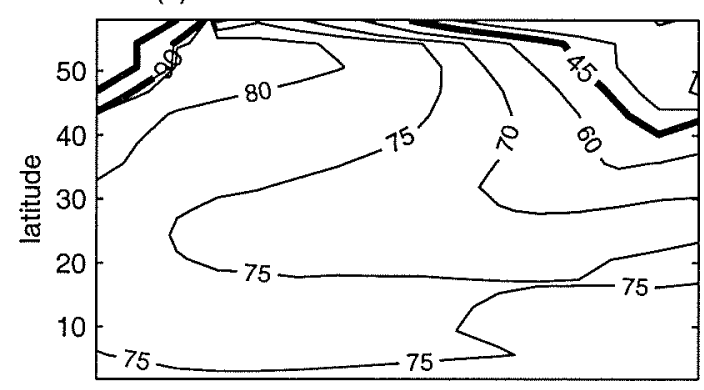

(c)

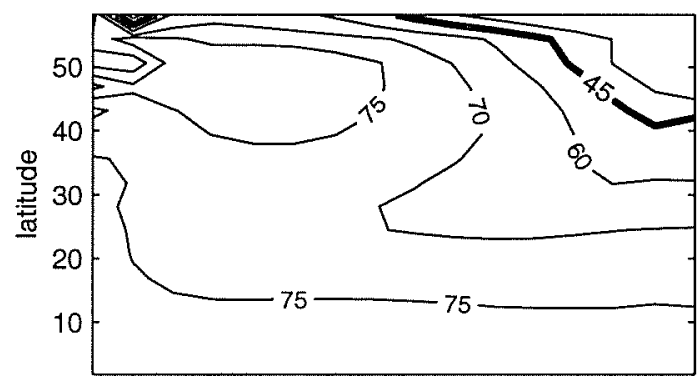

(e)

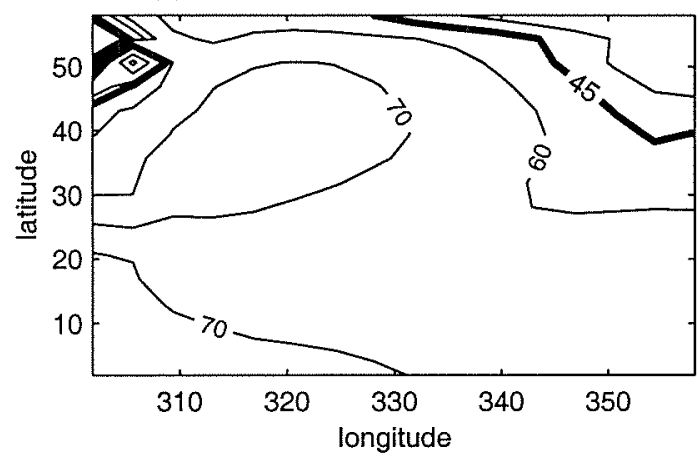

(b)

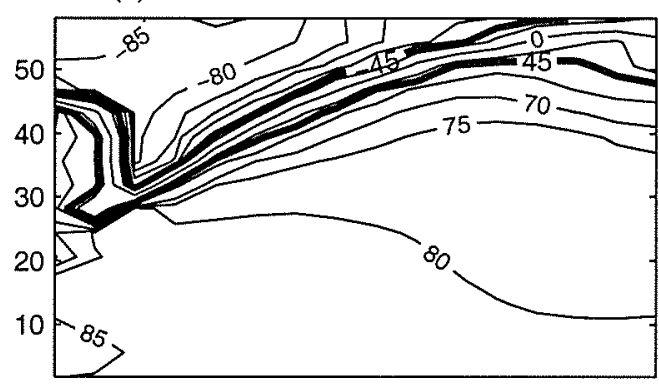

(d)
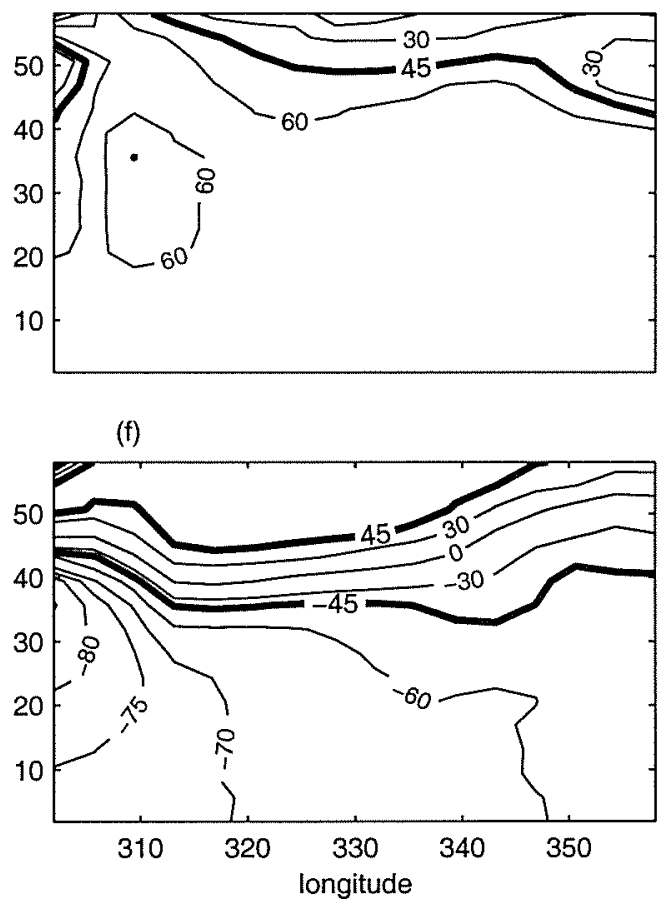

Figure 4.5: The Turner Angle at the bottom of level 7 (950m) for run (a) DDP (c) GHD (e) CDD and at the bottom of level $13(3500 m)$ for run (b) DDP (d) GHD and (f) CDD respectively. 
The density ratio is the most important index for double-diffusive activity. It controls the intensity of double diffusion and ultimately the large scale thermohaline circulation and water mass formation (see next). For both double-diffusive runs, we found that the distribution of density ratio in the upper kilometer at $24^{\circ} \mathrm{N}$ was in better agreement with the hydrographic data for the Atlantic as reported by Schmitt (1990) than the constant diffusivity run. However, the improvement was small, since the relaxation boundary conditions play a dominant role in setting upper ocean T-S structure. We find that the addition of double diffusion causes the density ratio to become closer to one, which is somewhat counter-intuitive (Figure 4.5). This means that double-diffusive processes and large scale circulation interact in a complicated way. Double diffusion in an one-dimensional (vertical) situation cannot be self-enhancing because of the weakening of the destabilizing gradient, which would shift $R_{\rho}$ away from one (Schmitt, 1981). However, in a two or three-dimensional model, double-diffusive activity appears able to reinforce itself. The double-diffusive lateral intrusions described by Turner (1978), Ruddick and Turner (1979) and Ruddick (1992) are a clear example of such self-reinforcing flows. That is, the maintenance and intensification of the double-diffusively favorable density ratio is due to the action of vertical shear on isopycnal gradients. The shear arises from horizontal pressure gradients developed when the double-diffusive fluxes begin affecting the vertical density profile. Whereas thermohaline intrusions have vertical scales of $100 \mathrm{~m}$ or less, it appears that such effects occur with much larger vertical scale within our model as well, based on the comparison of the DDP and CDD runs. As there are 1000-m vertical scale water masses in the ocean with similarly low density ratios, it would be interesting to determine how much of it is due to self-enhancing effects versus the juxtaposition of shear and isopycnal gradients due to the wind and buoyancy driven circulations.

Another speculation to explain the decrease of the density ratio with the implementation of double diffusion is the differences in $K_{T}$ and $K_{S}$. If we integrate the tracer equations over an isopycnal surface, the major steady-state balance is $w T_{z}=K_{T} T_{z z}$. Thus we have a solution of $T=T_{1}+T_{0} e^{\alpha_{T} z}$, and similarly $S=S_{1}+S_{0} e^{\alpha_{S} z}$, where $\alpha_{T}=w / K_{T}$, and $\alpha_{S}=w / K_{S}$. Assuming both $\alpha_{T}$ and $\alpha_{S}$ are constants, the density ratio is

$$
R_{\rho}=\frac{\alpha T_{0}}{\beta S_{0}} \frac{K_{S}}{K_{T}} e^{\left(\alpha_{T}-\alpha_{S}\right) z}
$$


For the conventional diffusivity case, $K_{T}=K_{S}$, thus $R_{\rho}=\frac{\alpha T_{0}}{\beta S_{0}}$. When we consider the effects of salt fingering, even though $K_{S} / K_{T}>1, e^{\left(\alpha_{T}-\alpha_{S}\right) z}<1$ at main thermocline depths and thus $R_{\rho}<\frac{\alpha T_{0}}{\beta S_{0}}$ for a wide range of depths. Therefore the density ratio in the main thermocline becomes smaller with salt fingering parameterization. When we consider the more accurate parameterization of salt fingering in Chapter 3 , the reduction in the density ratio is found to be larger than in the GHD runs.

\subsubsection{Temperature and Salinity Distribution}

The double-diffusive parameterization is also found to affect the basin-wide water mass properties. Changes in the temperature and salinity distributions due to DDP can be seen from the horizontally averaged temperature and salinity profiles (Figure 4.6). Compared to run CDD, the basin-averaged temperature in run DDP and GHD is lower in the uppermost 200 meters while it is higher below 200 meters. On the other hand, the horizontally-averaged salinity in both DDP and GHD is higher for the whole water column. The difference from run CDD is similar for run GHD and DDP, where both are most significantly different in the main thermocline. On the other hand, the difference from CDD seems twice as large in run DDP than in run GHD for the whole depth. The basin averaged temperature is $6.069^{\circ} \mathrm{C}$ for run $\mathrm{DDP}, 5.973^{\circ} \mathrm{C}$ for run GHD and $5.937^{\circ} \mathrm{C}$ for run CDD, and the salinity is $33.749 \mathrm{psu}, 33.723 \mathrm{psu}$ and $33.711 \mathrm{psu}$ respectively; thus, water in run DDP is $0.13^{\circ} \mathrm{C}$ warmer and $0.04 \mathrm{psu}$ saltier than in run CDD, while it is $0.04^{\circ} \mathrm{C}$ warmer and $0.01 \mathrm{psu}$ saltier in run GHD compared to run CDD.

Tracer distributions are controlled by advection and diffusion. While the wind-driven horizontal advection plays a prominent role in setting up the tracer distribution in the upper ocean, tracer distributions below the main thermocline are dominated by vertical advection and vertical diffusion with the horizontal advection playing a minor role only.

Under the relaxation boundary conditions for both temperature and salinity, surface temperature and salinity are nearly fixed to their climatological mean values. Since the salinity diffusivity in run DDP and GHD is larger than in run CDD, salinity is 
(a)

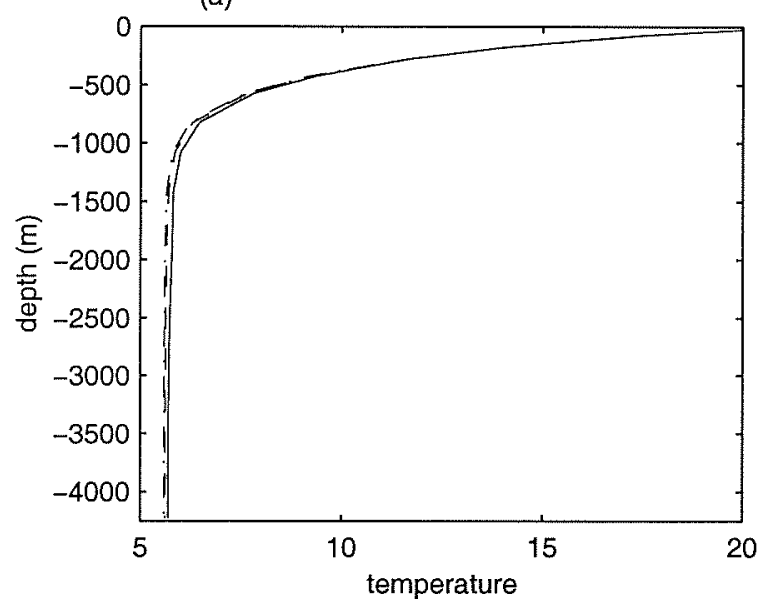

(c)

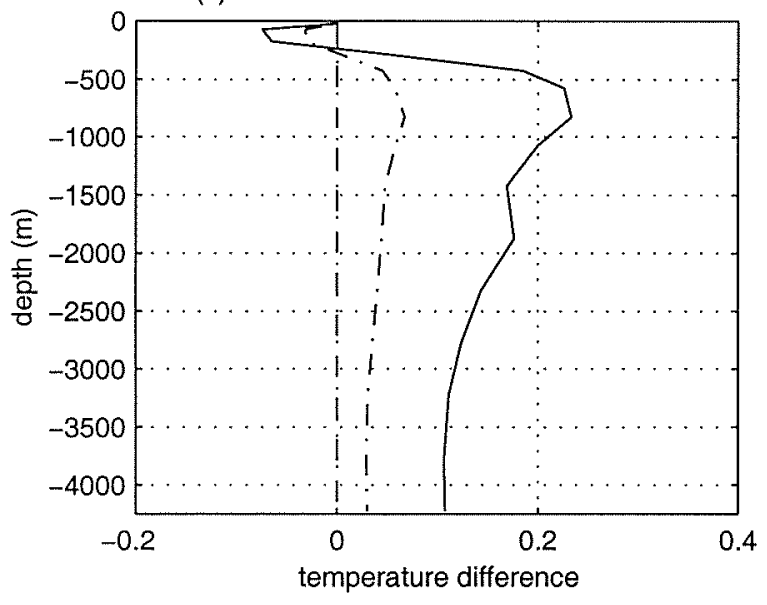

(b)

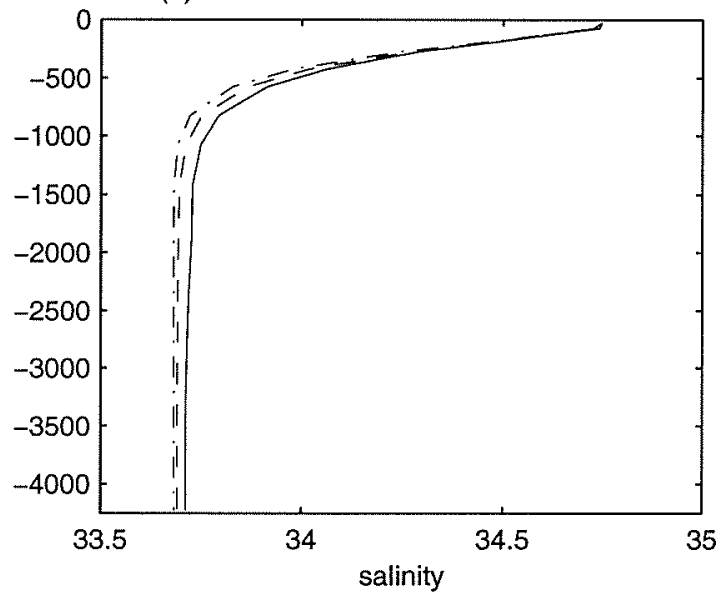

(d)

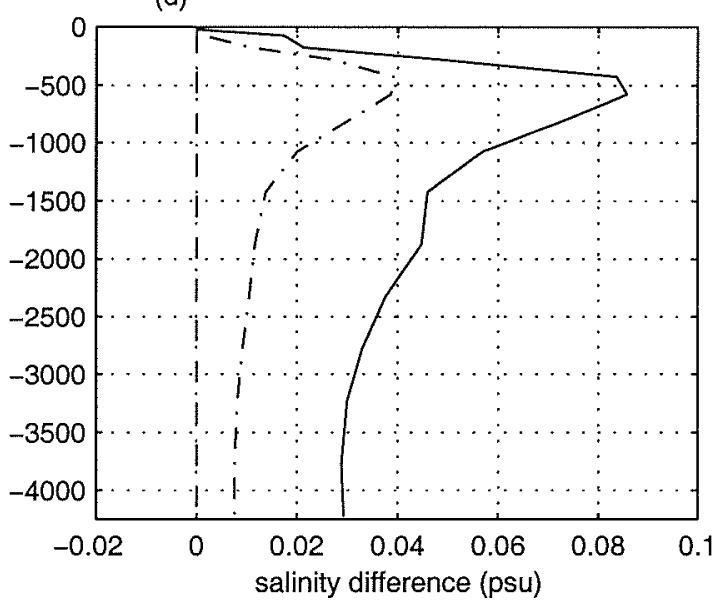

Figure 4.6: Vertical profiles of horizontally averaged (a) temperature and (b) salinity respectively, where the solid line for run DDP, the dotted broken line for run GHD and the broken line for run CDD. The difference from run CDD of (c) temperature and (d) salinity respectively. 
higher due to stronger downward salt diffusion in both DDP and GHD, compared with CDD, as shown in Figure 4.6.

Changes in the temperature profile can be explained by a simple one-dimensional advection-diffusion balance, neglecting the lateral advection and diffusion terms. Thus, the tracer distribution is controlled by the following one-dimensional balance $w T_{z}=\kappa T_{z z}$, where $z$ the distance along the path connecting the cold source and the hot source. Assuming both $w$ and $\kappa$ are constant along the path, the solution of this one-dimensional equation is in the form of $T=T_{1}+C\left(T_{2}-T_{1}\right)\left(e^{\alpha z}-1\right)$, where $C=\left(e^{\alpha D}-1\right), D$ is the distance between the cold and hot source, and $\alpha=w / \kappa$ is the inverse of the characteristic scale of this advection-diffusion problem. Small $\alpha$ means a diffusion-dominated case, with an almost linear profile between the cold and hot source, while a large $\alpha$ indicates a sharp front, like the main thermocline.

Salt mixing (circulation) is stronger (weaker) in run DDP and GHD, compared with CDD. Thus, the salinity profile in these two cases should have a broad halocline and high salinity in the abyssal water. From this solution, it is clear that strong diffusion and weak circulation give rise to a broad main thermocline, and warm and salty abyssal water, as shown in Figure 4.6.

The temperature profile is slightly more complicated to explain. In run DDP, strong mixing above the main thermocline transports more heat downward, so temperature is higher in the main thermocline, as shown in Figure 4.6. Warm water in the main thermocline makes the water in the whole basin warmer than in run CDD, even though thermal diffusion below the main thermocline in run DDP is weaker than in run CDD. The shallow cold temperature anomaly in run DDP reflects the fact that thermal diffusion near the surface is lower than average.

The temperature profile in run GHD is the result of a delicate balance between a weakened circulation and weakened diffusion. Compared with run CDD, the circulation in run GHD is $10 \%$ weaker, and diffusion is $5 \%$ weaker. Thus, the characteristic scale $1 / \alpha$ is reduced, and diffusion becomes more important than advection. As a result, the main thermocline is slightly broader, and thus the warm anomaly compared with run CDD, as shown in Figure 4.6. 


\subsection{Sensitivity Experiments}

Although the parameterization of double diffusion used here is consistent with existing theoretical, laboratory and observational results, the parameterization involves several free variables. Therefore, it is important to test the sensitivity of the model runs to these variables as well as other specifics of the numerical model, such as the vertical resolution. Note that all variables in these experiments are the same as in run DDP unless stated otherwise.

\subsubsection{Parameter Sensitivity}

There are four variables in the parameterization given in Chapter 3 for salt fingering: $K^{*}, K^{\infty}, R_{c}, n$. One special feature of this parameterization is that the diapycnal mixing consists of two parts: one part accounts for the double-diffusive processes, and the other part, $K^{\infty}$, represents the conventional, non-double-diffusive mixing due to turbulence (from internal wave breaking). The magnitude of both $K^{*}$ and $K^{\infty}$ are not firmly established and it is worthwhile to examine the model sensitivity to the ratio of $K^{*} / K^{\infty}$. For simplicity, we fix $K^{\infty}=0.3 \mathrm{~cm}^{2} \mathrm{~s}^{-1}$ and change the value of $K^{*}$.

This background diffusivity is higher than the value inferred from the tracer release experiment of Ledwell et al. (1993), which suggested a diffusivity of $0.15 \mathrm{~cm}^{2} \mathrm{~s}^{-1}$ in the main thermocline (which may be partially explained by salt fingers). As Yin and Fung (1991) have shown, unphysical vertical mixing in models with uneven vertical grids may occur, if the diffusivity is low, and this contaminates the numerical results. In order to avoid such numerical problems in our low resolution experiments, we have used a slightly high value of $0.3 \mathrm{~cm}^{2} \mathrm{~s}^{-1}$, which represent a compromise among the affordable vertical resolution, isopycnal mixing rate, and the observations.

Therefore we only need to test three variables, and the experiments and results are summarized in Table 4.1. Also, these results have been included in Figure 3.2, which are consistent with the scaling analysis.

From Table 4.1 we can see that different variable values lead to very different results. When we increase $K^{*}$, i.e, the contribution from double-diffusion, both $\bar{K}_{T}$ and 
Table 4.1: Sensitivity Experiments and Results .

\begin{tabular}{c|c|c||c|c|c}
\hline \hline$K^{*}\left(\mathrm{~cm}^{2} \mathrm{~s}^{-1}\right)$ & $R_{c}$ & $n$ & THC (Sv) & $\overline{K_{T}\left(\mathrm{~cm}^{2} \mathrm{~s}^{-1}\right)}$ & $\overline{K_{S}\left(\mathrm{~cm}^{2} \mathrm{~s}^{-1}\right)}$ \\
\hline 1.0 & 1.6 & 6 & 4.793 & 0.326 & 0.366 \\
\hline \hline 0.0 & 1.6 & 6 & 5.578 & 0.300 & 0.300 \\
\hline 0.2 & 1.6 & 6 & 5.505 & 0.304 & 0.310 \\
\hline 0.5 & 1.6 & 6 & 5.137 & 0.311 & 0.329 \\
\hline 2.0 & 1.6 & 6 & 4.156 & 0.350 & 0.426 \\
\hline \hline 5.0 & 1.6 & 6 & 3.475 & 0.379 & 0.506 \\
\hline 1.0 & 1.2 & 6 & 5.245 & 0.305 & 0.313 \\
\hline 1.0 & 2.0 & 6 & 3.935 & 0.356 & 0.441 \\
\hline \hline 1.0 & 1.6 & 2 & 3.759 & 0.353 & 0.436 \\
\hline 1.0 & 1.6 & 12 & 5.252 & 0.310 & 0.323 \\
\hline 1.0 & 1.6 & 32 & 5.310 & 0.306 & 0.313 \\
\hline 2.0 & 1.2 & 6 & 5.176 & 0.327 & 0.351 \\
\hline 0.5 & 2.0 & 6 & 4.650 & 0.329 & 0.375 \\
\hline \hline
\end{tabular}

$\bar{K}_{S}$ increase in a quasi-linear fashion, and the increase of double-diffusive processes decreases the diapycnal diffusion of density. Thus the magnitude of the meridional overturning is reduced. Similarly when we increase $R_{c}$ or decrease $n$, the mean diffusivity for heat and for salt increases, and the thermohaline circulation weakens.

\subsubsection{Sensitivity to Restraint $T_{z, c}$}

Experiments and results with different $T_{z, c}$ are summarized in Table 4.2.

The magnitude of $T_{z, c}$ can make a significant difference in the basin averaged diffusivities for salt and heat, while the magnitude of the meridional overturning remains nearly the same. The reason is that the effect of the constraint $T_{z, c}$ works like a filter, it only affects the part of ocean below the main thermocline where the vertical gradient of temperature and salinity is weak. In the main thermocline, however, $T_{z}$ is much larger than the $T_{z, c}$ given in the above table and thus the double diffusion in 
Table 4.2: $T_{z, c}$ Sensitivity Experiments and Results

\begin{tabular}{c||c|c|c}
\hline \hline$T_{z, c}\left(10^{-6}{ }^{\circ} \mathrm{Cm}^{-1}\right)$ & THC $(\mathrm{Sv})$ & $\bar{K}_{T}\left(\mathrm{~cm}^{2} \mathrm{~s}^{-1}\right)$ & $\bar{K}_{S}\left(\mathrm{~cm}^{2} \mathrm{~s}^{-1}\right)$ \\
\hline 0.0 & 4.733 & 0.386 & 0.476 \\
\hline 1.0 & 4.749 & 0.337 & 0.389 \\
\hline 2.5 & 4.811 & 0.326 & 0.366 \\
\hline 5.0 & 4.861 & 0.323 & 0.360 \\
\hline 10.0 & 4.944 & 0.318 & 0.348 \\
\hline \hline
\end{tabular}

the main thermocline remains almost the same. As found by Cummins et al. (1990), an increase of diffusivity in the deep ocean (they use a diffusivity which is inversely dependent on the local buoyancy frequency) increases the vertical gradient of $T, S$ in the deep ocean, but the magnitude of the thermohaline circulation is barely changed.

The criterion of a minimum $T_{z, c}$ for double diffusion to occur is speculative. We viewed it as a way to avoid spuriously large double-diffusion in the weakly stratified abyss, where there is little evidence for its importance, and noise in the computation of vertical gradients might be a problem.

Even though the double-diffusive activity in the deep ocean does not affect the magnitude of thermohaline circulation much, it can make a significant difference in the distribution of temperature and salinity. Compare run $T_{z, c}=0.0$ to a run with $K_{T}=K_{S}=0.43 \mathrm{~cm}^{2} \mathrm{~s}^{-1}$, i.e., the mean diffusivity in run $T_{z, c}=0.0$, the temperature is lower in the main thermocline $(0-500 \mathrm{~m})$ and warmer below $500 \mathrm{~m}$ depth, and the salinity is higher for the whole water depth. These changes in water mass properties caused by double diffusion are of interest for climate studies. We do not intend to compare results from our idealized runs with observations. However, we hope the underlying dynamics will apply to the more practical and complicated climate models. Danabasoglu and McWilliams (1995, DM hereafter) have tested the sensitivity of the global ocean circulation model to parameterizations of mesoscale isopycnal tracer transports, and they showed substantial improvement in several climatically important aspects of the ocean circulation, compared with the conventional horizontal/vertical eddy diffusion parameterization. However, the horizontally averaged temperature is warmer in the main thermocline and colder in the deep ocean compared with the Levitus climatology (Levitus, 1982) in their model (refer to Figure 8 in DM). The salinity is fresher throughout the water column, especially in the deep 
ocean (refer to Figure 16 in DM). These discrepancies are just the opposite of the changes we see in our $T_{z, c}=0$ run. Thus, our results suggest that the vertical temperature and salinity distributions could be improved in such global climate models if a parameterization of double diffusion were to be applied in the deep ocean. In this regard we note that McDougall and Whitehead (1984) invoked some salt fingering to explain the evolution of water mass properties in the Antarctic Bottom Water in the Atlantic.

\subsubsection{Salt Fingering and Diffusive Layering Alone}

To study the different roles played by salt fingering and diffusive layering in run DDP, we ran two experiments in which these two processes are separated with only one of them active in each experiment. For example, in the salt fingering alone run, the salt fingering parameterization (3.1-3.2) is activated, but diffusive layering is shut off, that is, a constant $K_{T}=K_{S}=K^{\infty}=0.3 \mathrm{~cm}^{2} \mathrm{~s}^{-1}$ is applied in places with no salt finger activity. The experiment for diffusive layering only is designed similarly.

In the salt fingering alone experiment, the meridional overturning rate is $4.80 \mathrm{~Sv}$, and the globally averaged diffusivities are: $\bar{K}_{T}=0.325 \mathrm{~cm}^{2} \mathrm{~s}^{-1}, \bar{K}_{T}=0.366 \mathrm{~cm}^{2} \mathrm{~s}^{-1}$, which is very close to run DDP. In contrast, in the diffusive layering alone experiment, the meridional overturning rate is $5.58 \mathrm{~Sv}$, and $\bar{K}_{T}$ and $\bar{K}_{S}$ are no more than $0.0003 \mathrm{~cm}^{2} \mathrm{~s}^{-1}$ higher than $K^{\infty}$. The global influence of diffusive layering activity is barely noticeable. Even though changes in $\bar{K}_{T}$ and $\bar{K}_{S}$ are tiny in this run, the meridional overturning rate is in fact enhanced, compared to the overturning rate of $5.575 S v$ for the case with $K^{*}=0$, as shown in Table 4.1. This result implies that diffusive layering may tend to intensify the thermohaline circulation. This might occur because the process can release heat to the upper ocean in the absence of deep convection, thereby converting a warm, salty water mass to a cold, salty water mass which more readily sinks. McDougall (1983) invokes such a mechanism for Greenland Sea Bottom Water formation. However, for our model, the effect is small, possibly because of the lack of an Arctic Ocean, where most of the diffusive layering is found (Padman and Dillon, 1988). 
Both experiments show that under restoring boundary conditions, salt fingering mixing occurs much more widely than diffusive layering. The additional constraint on $T_{z, c}$ could eliminate some places where diffusive layering is possible due to weak $T_{z}$, but the fingers predominate largely because of the restoring boundary conditions and the deep water formation at high latitudes, which produces cold, fresh deep waters. Thus, under such upper boundary conditions, diffusive mixing plays a small role in the diapycnal diffusion. In this case, the unphysical representation of diffusive layering by a simple $d=K_{S} / K_{T}>1$ in experiment GHD does not affect the result too much.

However, if we use a virtual salt flux condition or natural boundary condition (Huang, 1993), the situation should be different. Under the so-called "mixed boundary conditions", multiple equilibria exist. Convection could happen near the equator rather than in the polar region in the hemisphere model, or sinking near one pole while upwelling near another pole in a global model, thus the conditions favorable for diffusive layering are not affected in places where no convection occurs, and then it may be essential for the diapycnal mixing processes.

\subsubsection{Sensitivity to the Vertical Resolution}

The intensity of double-diffusive activity depends on the density ratio, $R_{\rho}=\alpha T_{z} / \beta S_{z}$, which involves the vertical gradient of temperature and salinity. Vertical temperature and salinity gradients may be poorly simulated in low-vertical resolution models. In addition, the observed vertical layer scale of staircases is smaller than the vertical grid scale (which produces the necessity for a parameterization). Thus, a natural question is how sensitive is the parameterization to the vertical resolution.

We ran an experiment with a doubled vertical resolution (compared to run DDP) and kept all other variables the same. The meridional overturning cell in this new experiment is $5.02 \mathrm{~Sv}$, and the basin-averaged diffusivities are $\bar{K}_{T}=0.337 \mathrm{~cm}^{2} \mathrm{~s}^{-1}, \bar{K}_{S}=$ $0.388 \mathrm{~cm}^{2} \mathrm{~s}^{-1}$. Compared to run DDP, it is clear that both the thermohaline circulation and basin-average mixing rates $\bar{K}_{T}, \bar{K}_{S}$ intensified.

The increase of the strength of the thermohaline circulation can be attributed to two aspects: (a) The increase of vertical resolution alone can increase the magnitude of 
the thermohaline circulation. We ran two experiments with constant $K_{T}=K_{S}=$ $0.45 \mathrm{~cm}^{2} \mathrm{~s}^{-1}$, one with 15 vertical levels and another with 30 levels. The meridional overturning rate was $7.47 \mathrm{~Sv}$ and $7.64 \mathrm{~Sv}$ respectively, a small but positive difference of 0.17Sv. We can expect a similar effect in the DDP experiments. (b) Even though the averaged $K_{T}, K_{S}$ are larger for the case with the higher vertical resolution, the strength of double diffusion in the main thermocline was actually slightly lowered. Since the increase in vertical resolution can increase the estimated vertical gradients of temperature and salinity (Weaver and Sarachik, 1990), especially in the deeper parts of the ocean, double diffusion can occur in this experiment at places where $T_{z}$ is lower than $T_{z, c}$ in the standard DDP run. Examination of the vertical distribution of diffusivities reveals that double diffusion reaches deeper in the experiment with higher vertical resolution than in the standard run. Thus, an increase in vertical resolution can improve the distribution of water masses, but the meridional overturning rate remains almost unchanged.

\subsection{Summary}

An investigation of the effects of double-diffusive mixing on the general circulation has been carried out using a more accurate parameterization than that used by Gargett and Holloway (1992), who applied a universal nonunity ratio between the vertical diffusivities of heat and salt.

Three experiments have been carried out using the GFDL MOM2 code based on standard relaxation boundary conditions for the surface temperature and salinity in a single-hemisphere ocean basin. The first run applied the above parameterization (DDP), the second run used the GH92 parameterization (GHD) and the third run used a constant diapycnal diffusivity (CDD). Compared to run CDD, the meridional overturning rate in run DDP is reduced by $22 \%$, and the poleward heat transport is reduced by $8 \%$. In comparison, the corresponding decrease in run GHD is $10 \%$ and $4 \%$ respectively. The density ratio distribution in run DDP and GHD is more favorable for double diffusion than in run CDD, indicating that changes in buoyancy fluxes affected the pressure field in a way which enhances the differential advection of heat and salt. Note that changes in density ratio distribution in run DDP are larger 
than in run GHD. Finally, deep temperatures and salinities become higher in run DDP and GHD than run CDD, and changes in run DDP are more than twice those in run GHD.

Results showed that the western boundary region is the most favorable place for double diffusion, due to the differential advection of water masses by strong surface and deep western boundary currents. This is consistent with the known occurrence of salt fingers in the western tropical Atlantic (Schmitt et al., 1987) and their inferred role in water mass changes in the Caribbean sea and Gulf of Mexico (Schmitz et al., 1993). Note however, given the simplicity of the model configuration, the distribution of density ratio and T-S properties is not comparable directly with the real oceans. Nevertheless, we believe that the dynamics of the interaction of vertically sheared advection and double diffusion applies to the real oceans.

Sensitivity experiments show that the values of $K^{*}, R_{c}, n$ can affect the ratio of the mixing due to double diffusion over the non-double-diffusive background mixing. When $K^{*}$ or $R_{c}$ are larger or $n$ is smaller, mixing due to double diffusion intensifies. As a result, diapycnal diffusion of buoyancy is reduced, and the thermohaline circulation rate declines. The additional constraint of $T_{z, c}$ on $T_{z}$ can affect the activity of double diffusion in the deep ocean and subpolar region, but it has no significant effect on the meridional overturning rate. Experiments with salt fingering only and diffusive layering only indicate that for a single-hemisphere basin under relaxation conditions for both temperature and salinity, salt fingering is dominant while diffusive layering activity is barely noticeable. However, diffusive layering could play a more important role under "mixed" type upper boundary conditions, and in models which include a polar ocean. Higher vertical resolution appeared to improve the distribution of water masses, but the strength of the thermohaline circulation remained about the same. From the limited observational data available, the parameterization proposed in Chapter 3 seems a reasonable first step to test the effects of double diffusion. Although we consider our parameterization to be rather conservative, its implementation led to significant changes in the circulation.

One of the more surprising results of this work was the finding that the introduction of double diffusion led to even more favorable conditions for its occurrence. Whereas in a one-dimensional simulation (Schmitt, 1981) double diffusion operates to drive $R_{\rho}$ away from one, its application in this general circulation model tended to generate even 
more areas with low density ratio. This is due to the double-diffusive redistribution of buoyancy, which produces pressure gradients that drive vertical shears that act on the isopycnal temperature and salinity gradients (Schmitt, 1990). Such self-enhancing effects are well recognized in finescale thermohaline intrusions, but our results indicate that they operate on larger vertical scales as well.

These experiments with the "standard" relaxation boundary conditions are intended to presage an examination of double-diffusive effects in circulation models with mixed boundary conditions. However, even the present results, which reveal a reduction in the meridional overturning circulation, should motivate work toward an improved parameterization of the double-diffusive mixing processes. 


\section{Chapter 5}

\section{Impacts of Double-diffusive}

\section{Processes on Thermohaline}

\section{Circulation under Mixed Boundary}

\section{Conditions}

\subsection{Introduction}

In Chapter 2, we have discussed the significance of the upper boundary conditions on the thermohaline circulation with the conventional uniform vertical mixing. Relaxation boundary conditions have been widely used in climate simulations, which force sea surface temperatures and salinities very close to observations. However, it is not physically justified to relax the sea surface salinity to the observations. Instead, a virtual salt flux boundary condition or freshwater boundary condition (Huang, 1993) for salinity should be used. Also, with mixed (or natural) boundary conditions, the thermohaline circulation displays multiple equilibria and variability on different time scales (Stommel 1961; Weaver and Hughes, 1992). One example of climate variability that occurred during the transition from the last glacial to the present inter-glacial 
period is the Younger Dryas cold event (about 11,000 to 10,000 years before present). Keigwin et al. (1991) documented that during the Younger Dryas, NADW production was substantially reduced or even eliminated, tending to support the hypothesis of Broecker et al. (1985) regarding the multiple equilibria of the "conveyor belt" circulation. In this chapter, the impacts of double-diffusive processes on the thermohaline circulation are investigated under mixed boundary conditions, i.e., a virtual salt flux condition is applied for salinity while temperature is relaxed to prescribed values.

Double-diffusive processes have a significant impact on the thermohaline circulation under relaxation boundary conditions (Zhang et al., 1998a). In Chapter 2, we found that under mixed boundary conditions, the stability, variability, and the existence of the multiple equilibria depends on diapycnal mixing process. Since the implementation of double-diffusive parameterization affects the diapycnal mixing (Chapter 3), we expect that the behavior of the thermohaline circulation will be different if we implement double-diffusive processes. Previous work by Gargett and Ferron (1996) investigated the effects of differential vertical diffusion of $T$ and $S$ in a box model of thermohaline circulation. They found that when $K_{T} \neq K_{S}$, the model exhibits extended ranges of multiple equilibria, a different mode transition near present-day values of freshwater forcing magnitude, and the possibility of quasi-periodic oscillatory states.

The organization of this Chapter is as follows. Model configuration and numerical experiments are introduced in Section 5.2. In Section 5.3, we investigate the response of the thermohaline circulation to changing freshwater forcing through quasi-equilibrium experiments. Sensitivity to variables in the parameterization of salt fingering is also investigated. In Section 5.4, individual experiments with fixed freshwater forcing are implemented, in different freshwater forcing regimes. A summary follows in Section 5.5 .

\subsection{Model Configuration}

In this Chapter, we use the $2 \beta$ version of GFDL Modular Ocean Model, in contrast with the $2 \alpha$ version used in previous chapters. In our experiments the only difference is the inclusion of the new implementation of Gent and McWilliams (1990, GM90) 
isopycnal mixing scheme proposed by Griffies et al. (1998), in which they showed that the original GM90 parameterization code is numerically unstable in some regions. They refined the GM90 parameterization to satisfy the following two constraints: (a) down-gradient orientation of the diffusive fluxes along the neutral surface direction (b) zero isoneutral diffusive flux of local potential density.

Temperature is relaxed to the following linear profile:

$$
T=T_{0}\left(1-\phi / \phi_{N}\right)
$$

where $T_{0}=25^{\circ} \mathrm{C}, \phi$ is latitude and $\phi_{N}$ is the most northern grid latitude. A virtual salt flux condition is used for salinity. The profile of the freshwater forcing will be discussed next. To be consistent with the scaling analysis in previous chapters and to focus on the buoyancy-driven ocean circulation, wind stress is not present.

$e-p$ profile

Even though (2.107) gives a simple profile of $e-p$, it has more evaporation than precipitation in the equatorial regions, which is contrary to observations. Here we use a new profile that is more realistic compared with the North Atlantic climatology:

$$
e-p=-W_{0} \cos (5 \phi) / \cos \phi
$$

This $e-p$ profile is plotted in Figure 5.1, together with the zonally averaged $e-p$ data from Schmitt et al. (1989). Note that the $e-p$ from Schmitt et al. (1989) is modified so that the total $e-p$ in the model region $0^{\circ} N-60^{\circ} \mathrm{N}$ is zero. We can see that the fit function given in (5.2) with $W_{0}=0.6 \mathrm{~m}_{\text {year }}{ }^{-1}$ roughly follows the meridional variation of the climatological data.

For comparison with the scaling analysis, in which the strength of freshwater forcing needs to be specified, we use

$$
E=1.5 W_{0}
$$

similar to relation (2.110), to represent the average of the maximum evaporation in the subtropical region and the maximum precipitation in the polar region. 


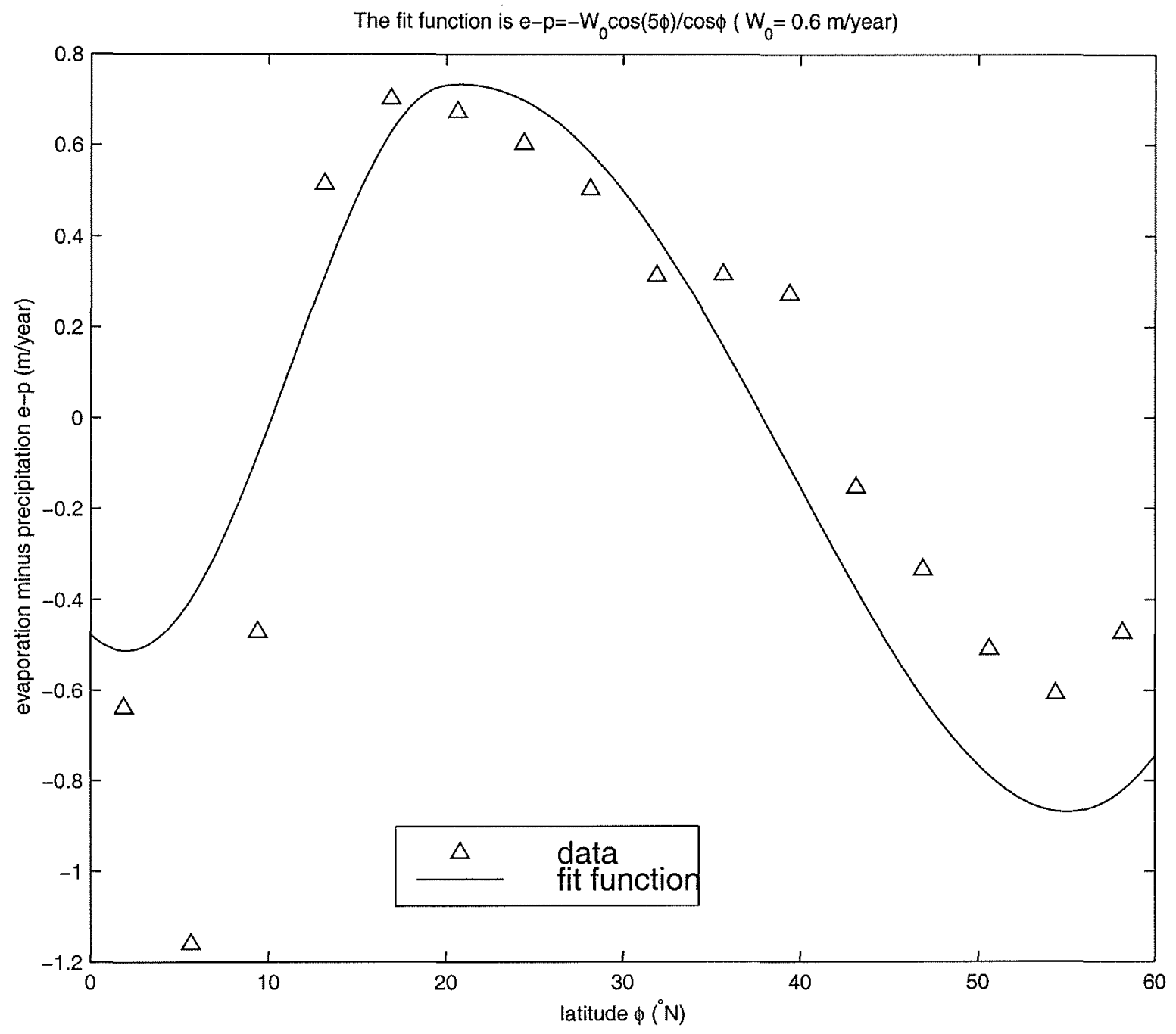

Figure 5.1: Fit function and climatology of the zonally averaged $e-p$ distribution in the North Atlantic. 


\section{Numerical Experiments}

Two types of experiments are performed under "mixed" boundary conditions: one type with constant diapycnal diffusivity (CDD):

$$
K_{T}=K_{S}=0.5 \mathrm{~cm}^{2} \mathrm{~s}^{-1},
$$

and another type with the double-diffusive parameterization (DDP) $(3.1-3.2)$, implemented with variables given as follows:

$$
\begin{aligned}
K^{*} & =2.0 \mathrm{~cm}^{2} \mathrm{~s}^{-1}, K^{\infty}=0.5 \mathrm{~cm}^{2} \mathrm{~s}^{-1}, \\
R_{c} & =1.6, n=6, T_{z, c}=2.5 \times 10^{-4}{ }^{\circ} \mathrm{Cm}^{-1}
\end{aligned}
$$

Note that the values of $K^{*}, K^{\infty}$ are slightly larger than (3.17), in order to reach steady state more efficiently. These values are still comparable with observations or the common values used in OGCMs. In addition, sensitivity to the above variables will be investigated.

\subsection{Quasi-Equilibrium Experiments}

One very interesting problem with mixed boundary conditions is that the existence of the multiple equilibria of the thermohaline circulation depends on the relative contribution of the freshwater forcing and diapycnal diffusivity. Since the doublediffusive processes can change the diapycnal diffusivity, we expect that the critical freshwater forcing will be affected. Here we adopt the quasi-equilibrium approach used in Chapter 2.

\subsubsection{Two Control Experiments}

Two experiments are performed, with the diapycnal diffusivities given in (5.4) and (5.5) respectively. Similar to section 2.4.4, we first run the model to an equilibrium with no freshwater forcing ( $W_{0}=0 \mathrm{~m}$ year $^{-1}$ ). From there we increased $W_{0}$ very slowly (0.05 $\mathrm{m}_{\text {year }}{ }^{-1}$ per thousand years). Due to the slow rate of change of the forcing, the model remains in quasi-equilibrium and we can track the quasi-equilibrium 
response of the model to the different freshwater forcings, with the thermal forcing basically fixed. The results are plotted in Figure 5.2. In comparison, we also included the scaling results in this figure, with variables as given in (2.63) except that $\alpha=1.5 \times 10^{-4} K^{-1}$.

From Figure 5.2, we can see that with the implementation of double-diffusive parameterization, the critical freshwater forcing that destabilizes the thermal mode decreases, consistent with the results from scaling analysis (heavy lines). This implies that the size of freshwater flux that leads to the cessation of the thermal mode is smaller with double-diffusive processes acting than in the conventional mixing case. When the freshwater forcing is relatively weak $\left(E<0.2 \mathrm{~m} \mathrm{year}^{-1}\right)$, the two experiments are almost identical. This is confirmed by the scaling analysis, which demonstrates that the density ratio in the ocean depends on the strength of the freshwater forcing. When the freshwater flux is weak, the density ratio is relatively high. The double-diffusive fluxes only become significant when the density ratio is small. As a result, the mixing due to double-diffusion can be neglected for weak freshwater forcing and the circulation is nearly identical to run CDD. In the quasi-equilibrium experiments when $E>0.2 \mathrm{~m}_{\text {year }}{ }^{-1}$, the meridional overturning in run DDP is smaller than run CDD, and the difference increases with larger freshwater forcing. At about $E=0.33$ mear $^{-1}$, the thermal mode cannot survive in run DDP. In the CDD run, the thermal mode circulation rate drops dramatically at about $E=0.40 \mathrm{~m} \mathrm{year}^{-1}$. The scaling predicted critical values of about $E=0.3$ and $0.42 \mathrm{~m}_{\text {year }}{ }^{-1}$ for the above two experiments. The two numerical experiments are consistent with the scaling analysis results. It is clear that the impacts of double-diffusive processes depend on the strength of the freshwater forcing. 


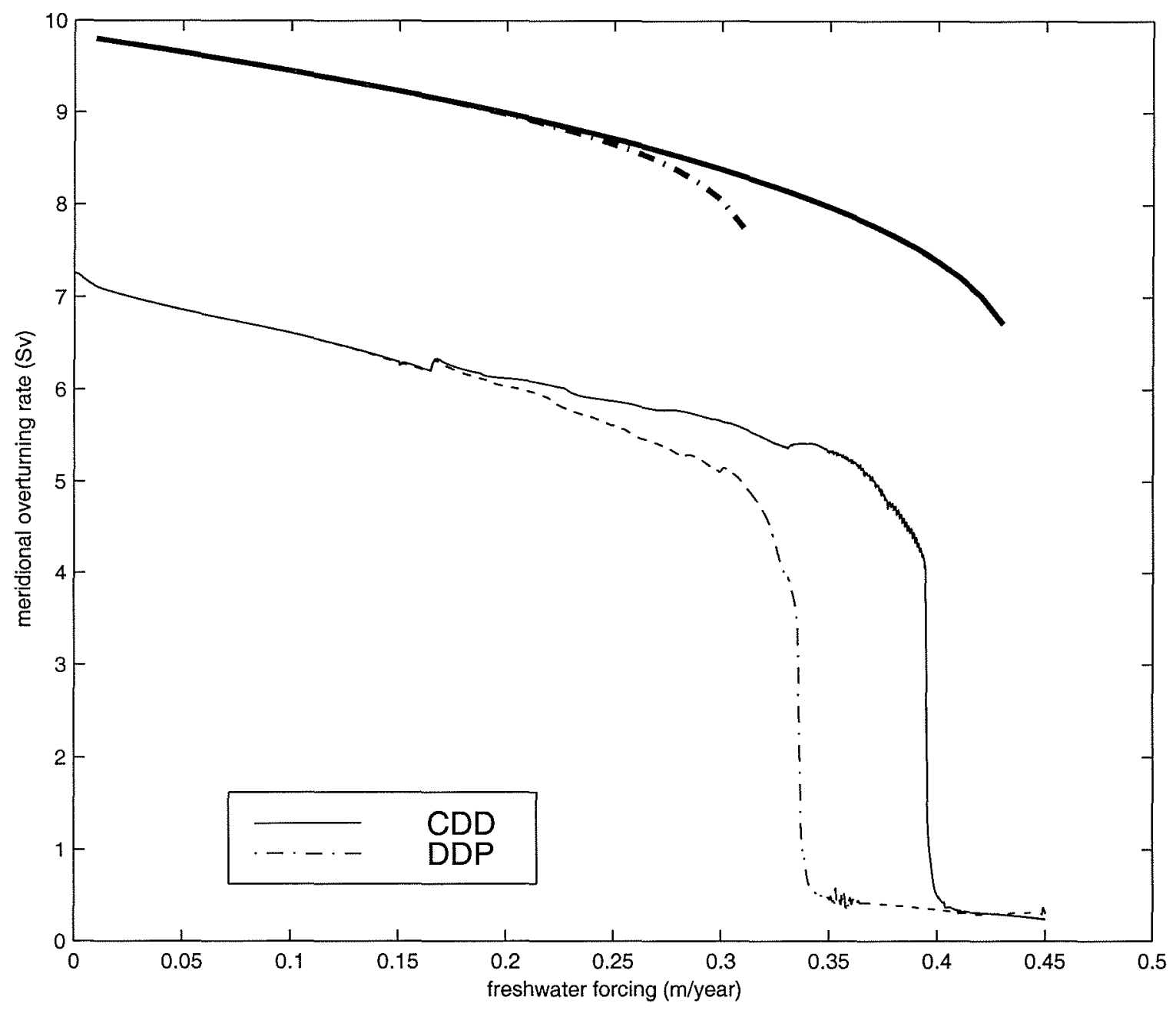

Figure 5.2: Change of the stable thermal mode with the freshwater forcing, where the heavy lines represent the result from scaling analysis and the thin lines for the quasi-equilibrium numerical experiments. 


\subsubsection{Parameter Sensitivity Experiments}

Given the uncertainties in the salt fingering parameterization, it is important to examine how sensitive the model is to the parameterization variables. In Chapter 3 , the parameter sensitivity was examined through scaling analysis; here we will investigate it through quasi-equilibrium numerical experiments.

Three experiments are implemented to compare with run DDP in last subsection (here we name it DDP_CN: the control experiment). The experiments and the corresponding variables are listed in Table 5.1. From this table, we can see that each experiment differs from DDP_CN only in one variable.

Table 5.1: Parameter Sensitivity Experiments

\begin{tabular}{c|c|c|c|c}
\hline \hline Experiment & $K^{*}\left(\mathrm{~cm}^{2} \mathrm{~s}^{-1}\right)$ & $R_{c}$ & $n$ & $K^{\infty}\left(\mathrm{cm}^{2} \mathrm{~s}^{-1}\right)$ \\
\hline DDP_CN & 2.0 & 1.6 & 6 & 0.5 \\
\hline DDP_K1 & 1.0 & 1.6 & 6 & 0.5 \\
\hline DDP_R2 & 2.0 & 2.0 & 6 & 0.5 \\
\hline DDP_n2 & 2.0 & 1.6 & 2 & 0.5 \\
\hline \hline
\end{tabular}

The numerical experiment results are plotted in Figure 5.3. For comparison, the results from scaling analysis are also plotted in Figure 5.3 (heavy lines). Again, the quasi-equilibrium numerical experiments are consistent with the scaling analysis. In run DDP K1, with the maximum diapycnal diffusivity due to salt fingers set to be half of that in run DDP_CN, the impact of salt fingers is substantially reduced. The critical freshwater forcing that stops the thermal mode is increased (though it is still larger than that in run CDD of last subsection). In run DDP_R2 and DDP_n2, the critical density ratio and exponent index are increased and reduced respectively, compared to run DDP_CN. Salt fingering in these runs has a larger effect for the same density ratio distribution, which can explain the reduction in the critical freshwater forcing in the above two experiments, as indicated by the scaling analysis.

The importance of the double-diffusive processes on the thermohaline circulation and the sensitivity to the uncertain variables in the parameterization of double-diffusion requires a better knowledge of these processes in the real ocean. 


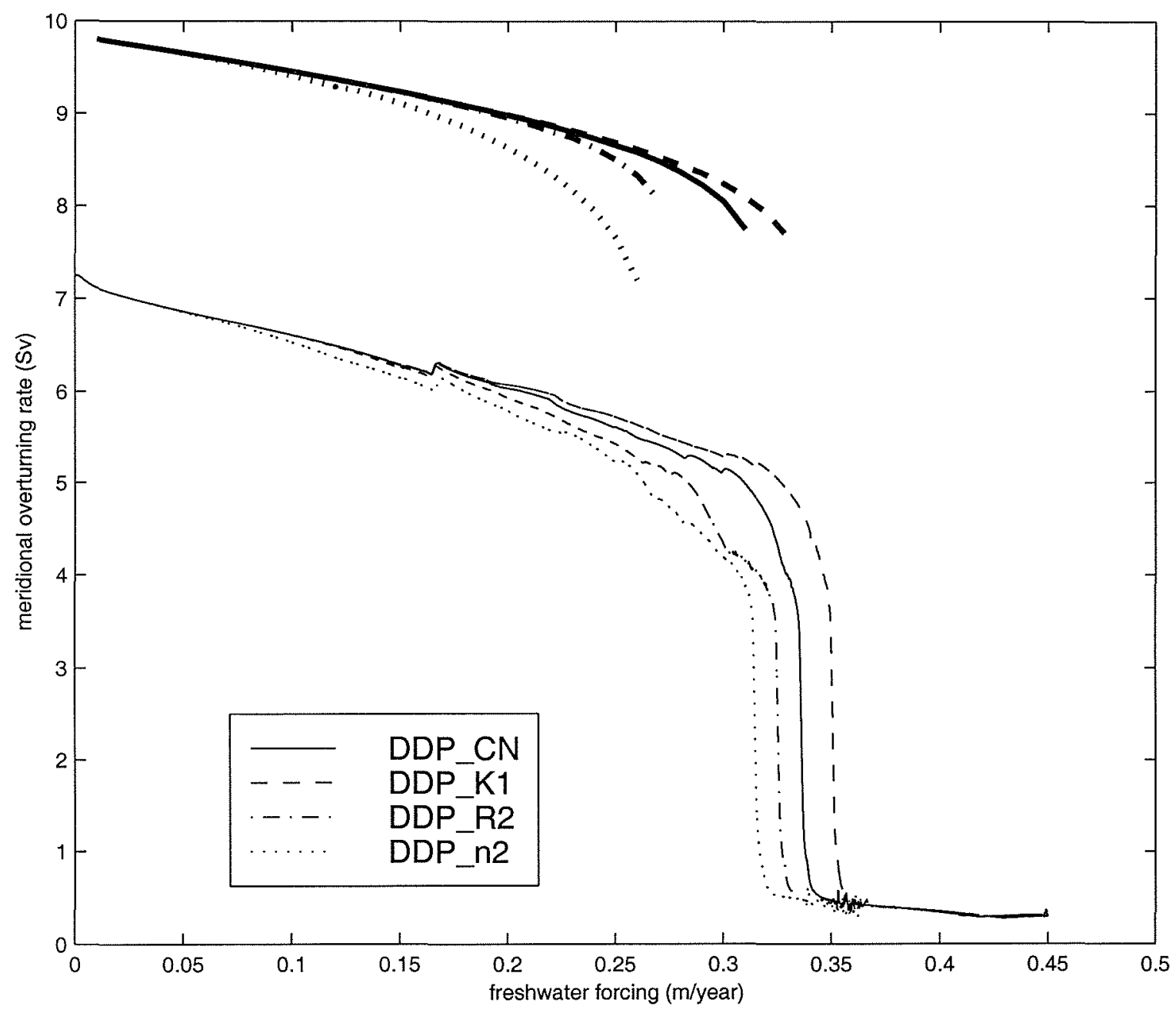

Figure 5.3: Sensitivity of quasi-equilibrium experiments to the variables in the parameterization of salt fingering. The heavy lines are results from scaling analysis and thin lines for numerical experiments. 


\subsection{Individual Experiments}

From the quasi-equilibrium experiments in last section, we found that the impact of double diffusion on the thermohaline circulation depends on the strength of the freshwater forcing. Here we will investigate the corresponding effects in different freshwater parameter regimes with freshwater forcing fixed for each experiment:

(a) weak freshwater forcing regime. Here "weak" means that the freshwater forcing is much less than the critical freshwater forcing. The thermohaline circulation lies in the thermal mode in this regime. We use $W_{0}=0.10 \mathrm{~m}_{\text {year }}{ }^{-1}$ (equivalent to $E=0.15 \mathrm{~m} \mathrm{year}^{-1}$ ) in the following experiments.

(b) intermediate freshwater forcing regime. Here "intermediate" means that the freshwater forcing is close to the critical value. We choose $W_{0}=0.20,0.25,0.30 \mathrm{~m}_{\text {year }}{ }^{-1}$ (equivalent to $E=0.30,0.375,0.45 \mathrm{~m}_{\text {year }}{ }^{-1}$ respectively).

(c) strong freshwater forcing regime. Here "strong" means that the freshwater flux is so large that the thermal mode cannot be sustained for both types of experiments and the thermohaline circulation has the characteristics of the haline mode. We choose $W_{0}=0.50,0.80 \mathrm{~m}_{\text {year }}{ }^{-1}$ (equivalent to $E=0.75,1.20 \mathrm{~m}_{\text {year }}{ }^{-1}$ respectively).

The numerical experiments for the above three regimes will be discussed separately.

\subsubsection{Weak Freshwater Forcing}

With $W_{0}=0.1 \mathrm{~m}_{\text {year }}{ }^{-1}$, two experiments are performed, one CDD and another DDP. After 5,000 years of integration, both experiments reached steady thermal mode solution.

\section{Meridional Overturning}

The meridional overturning cells for CDD and DDP are nearly identical, both with a maximum of $6.2 \mathrm{~Sv}$. The structure of the meridional overturning cell for run CDD is shown in Figure 5.4. 
There are similarities and differences in the structure of the thermohaline circulation between Figure 5.4 and that under relaxation boundary conditions (RBC, for example, Figure 4.2). Deep water is formed in the polar region and convected downward under both types of boundary conditions, and moves equatorward and upwells throughout the whole basin. Compared to that under $\mathrm{RBC}$, the maximum meridional overturning rate lies at a lower latitude (about $44^{\circ} \mathrm{N}$ compared with $50^{\circ} \mathrm{N}$ under RBC) and shallower depth (about $700 \mathrm{~m}$ deep rather than $2000 \mathrm{~m}$ deep under RBC).

\section{$R_{\rho}, K_{S}$ Distribution in run DDP}

With $W_{0}=0.1 m$ year $^{-1}$, the basin averaged $K_{T}$ and $K_{S}$ in run DDP are:

$$
\bar{K}_{T}=0.5036 \mathrm{~cm}^{2} \mathrm{~s}^{-1}, \quad \bar{K}_{S}=0.5136 \mathrm{~cm}^{2} \mathrm{~s}^{-1} .
$$

We see that $\bar{K}_{S}>\bar{K}_{T}$, indicates salt fingering is the dominant double-diffusive processes. Double diffusion appears to change $\bar{K}_{S}$ by less than $3 \%$ from the background value. It is not surprising that we don't see much difference in the magnitude and structure of the meridional overturning cell in run CDD and DDP.

The spatial variability of $R_{\rho}$ and $K_{S}$ in run DDP is illustrated in Figure 5.5. In the deep ocean, double diffusion is not activated since the vertical gradient of temperature and salinity is too weak. Thus we plot only the distribution in the upper 1500 meters. There are two major features:

(a) Similar to RBC, the density ratio in the western boundary current (WBC) region is smaller than in the interior, as a result, the eddy diffusivity of salinity is stronger in the WBC than in the interior. Therefore, the WBC is more favorable to salt fingers than the interior under mixed boundary conditions.

(b) There is a vertical variation in $R_{\rho}$, and thus in $K_{S}$ as well. The density ratio in the main thermocline is very high, and much smaller at the bottom of the main thermocline. This is because the density ratio in the main thermocline is set up by the meridional gradient of sea surface temperature and sea surface salinity. Since the later is proportional to the strength of the freshwater forcing, the weak freshwater forcing leads to a small meridional gradient of sea surface salinity, and therefore a 


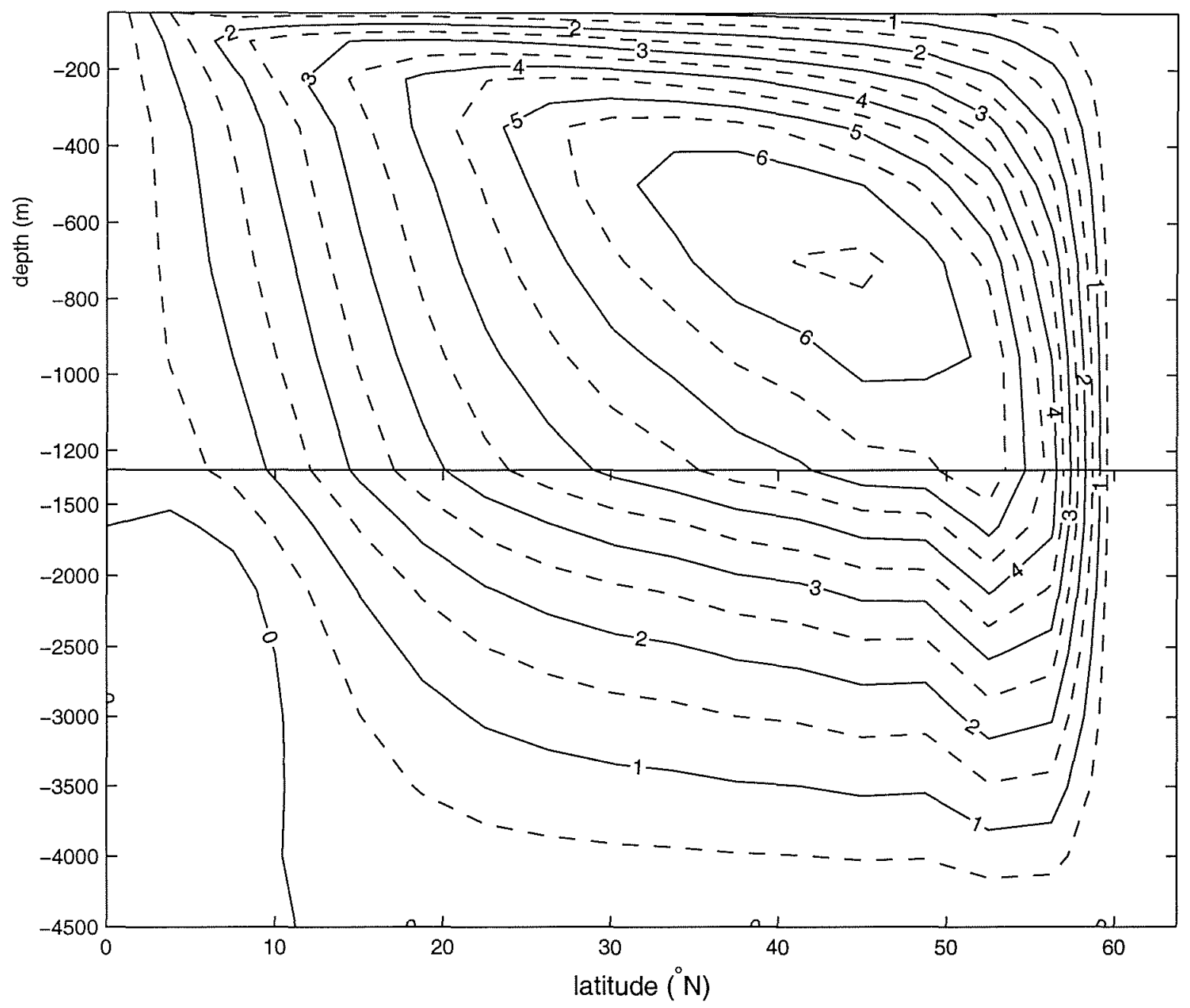

Figure 5.4: The meridional overturning with $W_{0}=0.1 \mathrm{~m}_{\text {year }}{ }^{-1}$ for CDD. The contour interval is $0.5 \mathrm{~Sv}$. 

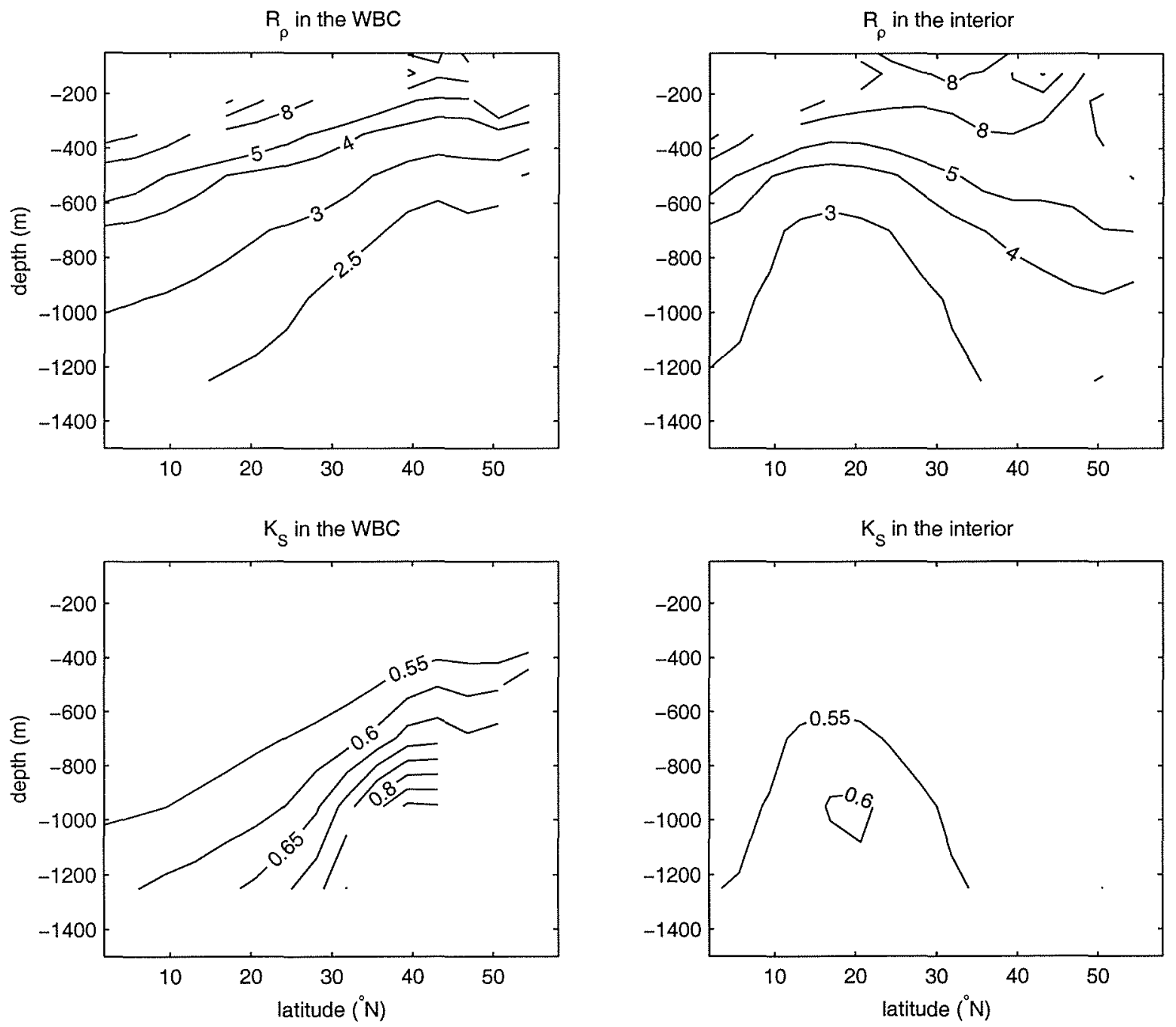

Figure 5.5: The distribution of $R_{\rho}$ (upper panels) and $K_{S}$ (lower panels) in the region of western boundary current (WBC) (left panels) and interior (right panels) respectively, in run DDP with $W_{0}=0.1 \mathrm{~m}_{\text {year }}{ }^{-1}$. Note that unequal contour intervals have been used for $R_{\rho}$, and the contour interval for $K_{S}$ is $0.05 \mathrm{~cm}^{2} \mathrm{~s}^{-1}$. 
large density ratio. In contrast, the density ratio below the main thermocline is more the result of the water mass formed in the polar region.

In the scaling analysis, we used the horizontal density ratio at the sea surface to represent the magnitude of the density ratio in the interior ocean. This scale works for the main thermocline but not for the abyss. However, since the magnitude of the thermohaline circulation is much more sensitive to the vertical mixing processes in the main thermocline than in the deep ocean (Cummins et al., 1990), we can still use the surface horizontal density ratio to measure the intensity of double diffusion as far as the strength and mode of the thermohaline circulation is concerned.

\section{Water Mass Structure}

Even though the double diffusion in run DDP is rather weak, making hardly any difference in the magnitude and structure of the thermohaline circulation, it does make some difference in the T-S structure (Figure 5.6). Compared to run CDD, the water in run DDP is colder and fresher in the thermocline, and warmer and saltier in the deep ocean. This is because the salt fingering is most intense at the bottom of the main thermocline (between $600 \mathrm{~m}-1200 \mathrm{~m}$ in Figure 5.5), which diffuses more heat and salt downward. On the other hand, the results here confirm those of Cummins et al. (1990) in that the increased diffusivity below the thermocline hardly affects the magnitude of the thermohaline circulation.

In general, however, the differences in the water mass properties is small between runs CDD and DDP, since the freshwater forcing is weak, and the intensity of salt fingers remains low, even at the bottom of the main thermocline where salt fingers were found to be most active. 

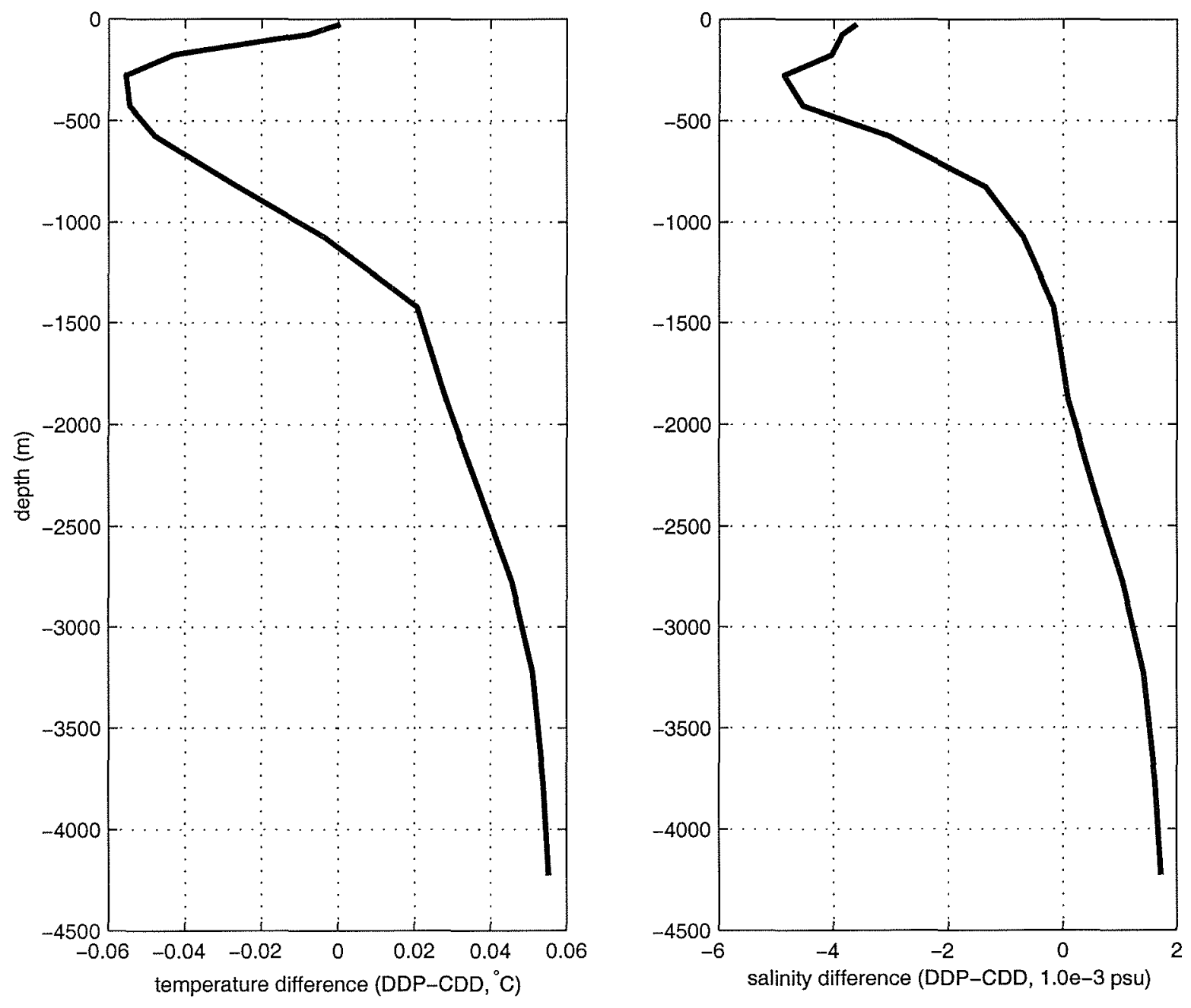

Figure 5.6: The temperature and salinity difference of run DDP from run CDD, with $W_{0}=0.1$ mear $^{-1}$. 


\subsubsection{Intermediate Freshwater Forcing}

\section{Meridional Overturning}

When $W_{0}=0.2$ mear $^{-1}$, the CDD and DDP experiments achieved a steady thermal mode after 4,000 years of integration. The meridional overturning cells for both runs are plotted in Figure 5.7. For this freshwater forcing, we see that implementation of the double-diffusive parameterization reduces the magnitude of the meridional overturning rate. The maximum streamfunction value in run CDD is $6.09 \mathrm{~Sv}$ whereas the maximum in run DDP is $5.64 \mathrm{~Sv}$, nearly an $8 \%$ reduction. The structure of the meridional overturning cell for runs CDD and DDP are similar.

Compared to the weak freshwater forcing case $\left(W_{0}=0.1 \mathrm{~m}\right.$ year $\left.{ }^{-1}\right)$, the magnitude of the thermohaline circulation is reduced in both types of experiments, indicating that under mixed boundary conditions the increased freshwater forcing leads to a stronger north-south surface salinity difference. North-south salinity difference is the "braking" force for the thermal mode, and therefore a weaker overturning rate. Also in contrast with the $W_{0}=0.1 \mathrm{~m}$ year $^{-1}$ case, the difference in the magnitude of the thermohaline circulation for run CDD and DDP is much larger, consistent with the scaling analysis and quasi-equilibrium numerical experiments (Figure 5.2).

When $W_{0}=0.25 \mathrm{~m}_{\text {year }}{ }^{-1}$, there is a large difference between runs CDD and DDP (the transient behavior of the meridional overturning rate is plotted in Figure 5.8 (a)). For run CDD, a steady thermal mode is reached. For run DDP, the thermal mode cannot be maintained and instead, convection occurs at about $20^{\circ} \mathrm{N}$ where the maximum in $e-p$ is located: characteristics of the so-called haline mode. For haline mode, "flushing" (Marotzke 1990; Huang 1994) occurs at about 3,700 years in run DDP.

When $W_{0}=0.3$ m year $^{-1}$, neither CDD nor DDP reach steady state (Figure $5.8(\mathrm{~b})$ ). Instead, both experiments entered the haline mode, which is associated with strong variability in the thermohaline circulation (Figure 5.8). Run CDD (solid heavy line) shows a regular flushing phenomenon, with a period of about 2500 years. Run DDP shows the flushing in a more chaotic manner. 
(a) $\mathrm{CDD}$

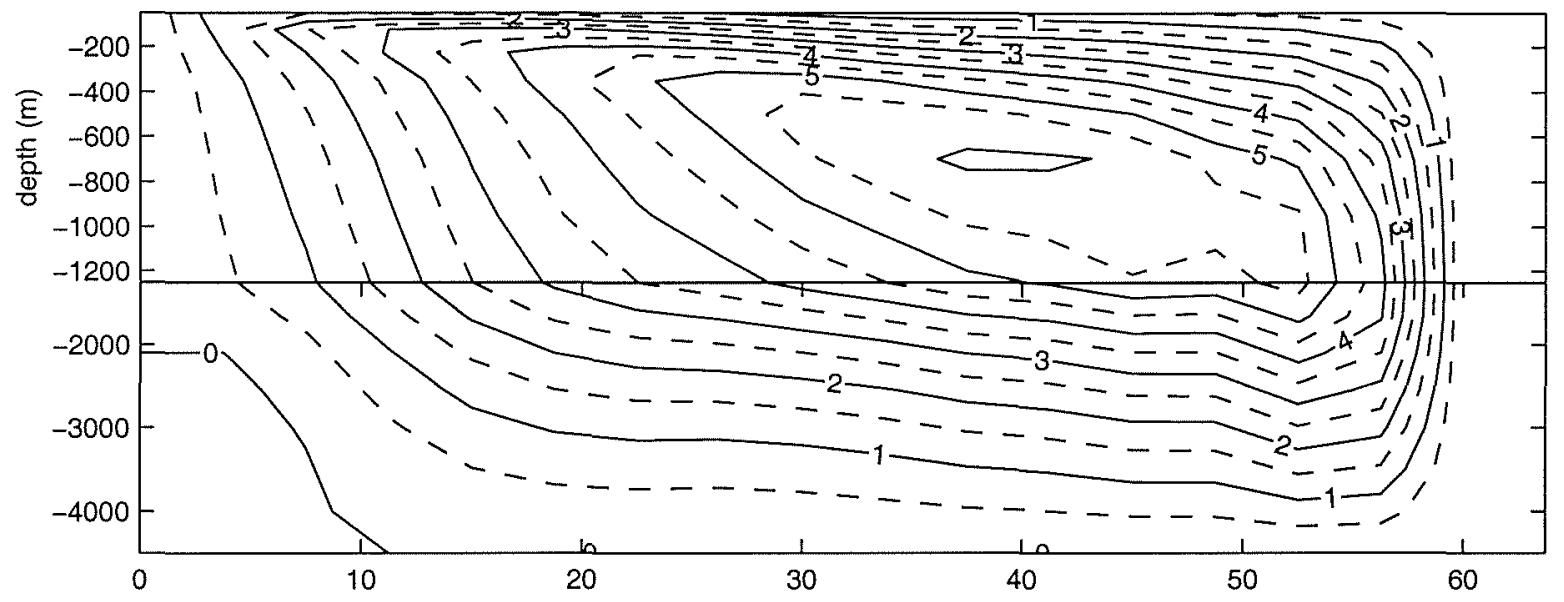

(b) DDP

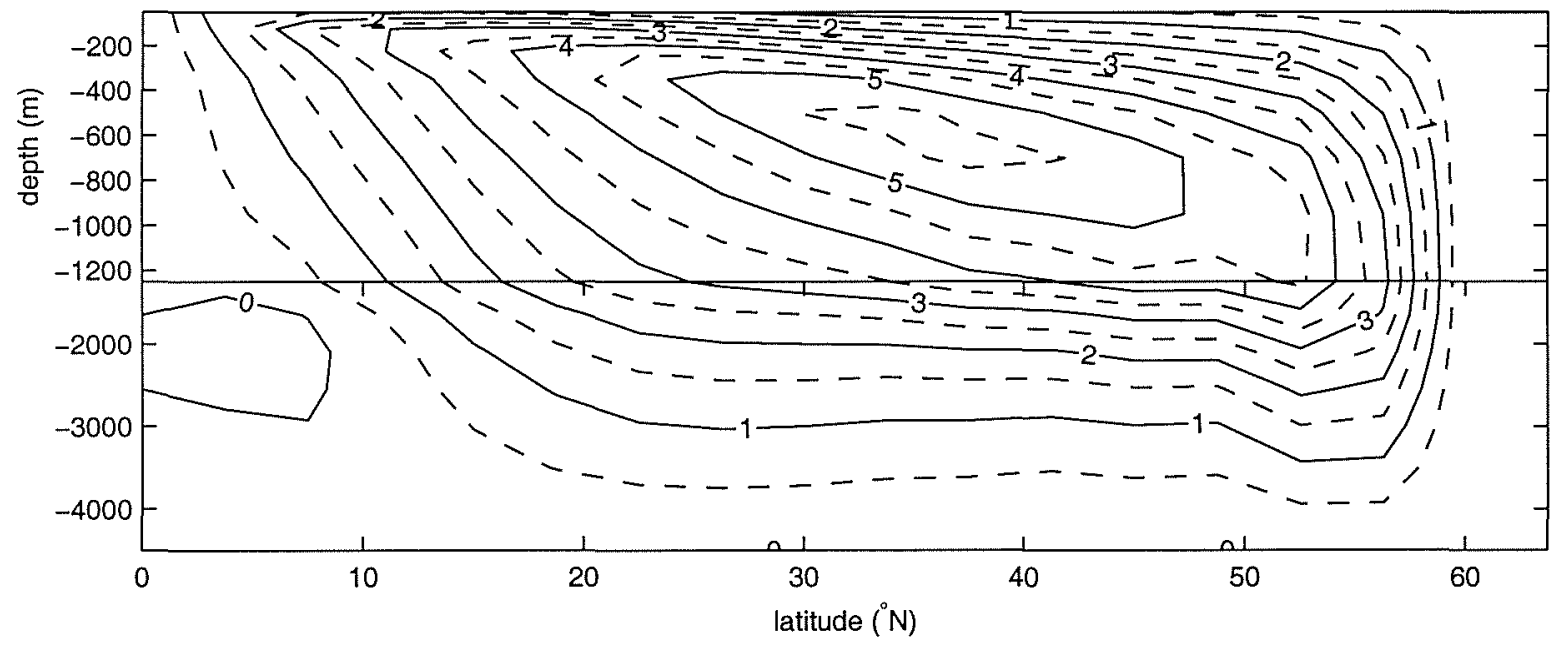

Figure 5.7: The meridional overturning with $W_{0}=0.2 m$ year ${ }^{-1}$. The upper panel is for CDD and the lower one for DDP. 
(a) $W_{0}=0.25 \mathrm{~m} /$ year

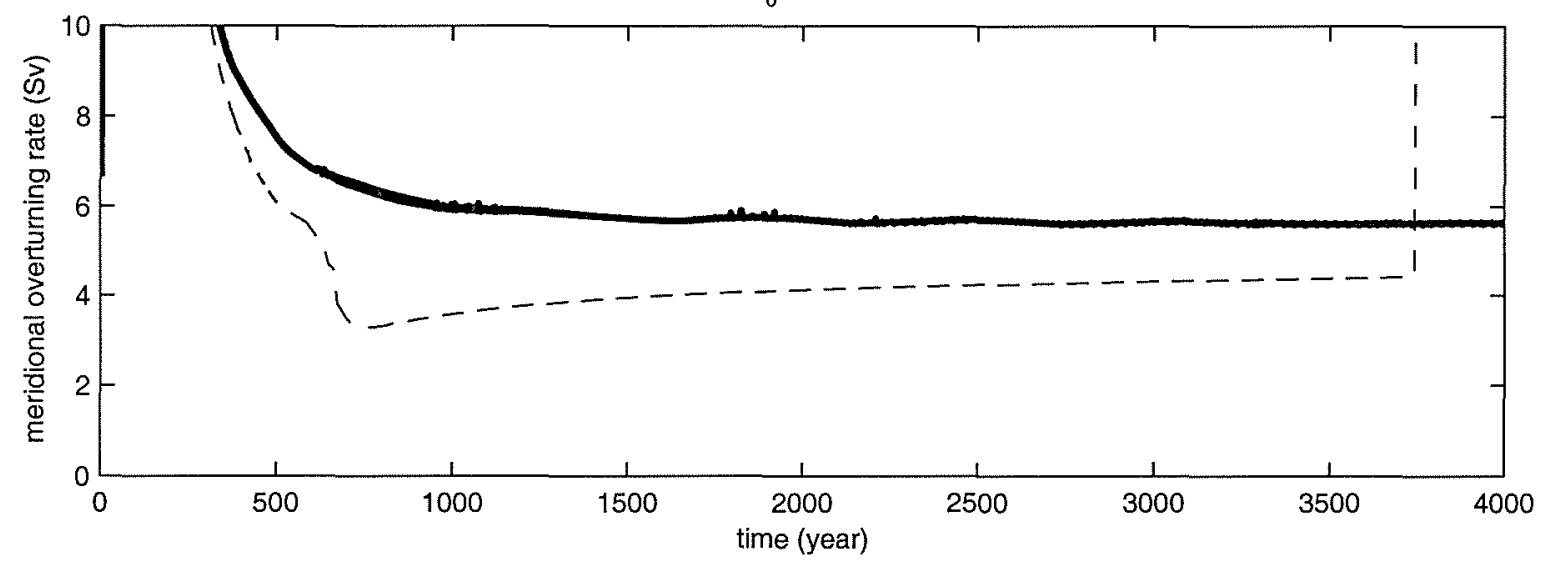

(b) $\mathrm{W}_{0}=0.3 \mathrm{~m} /$ year

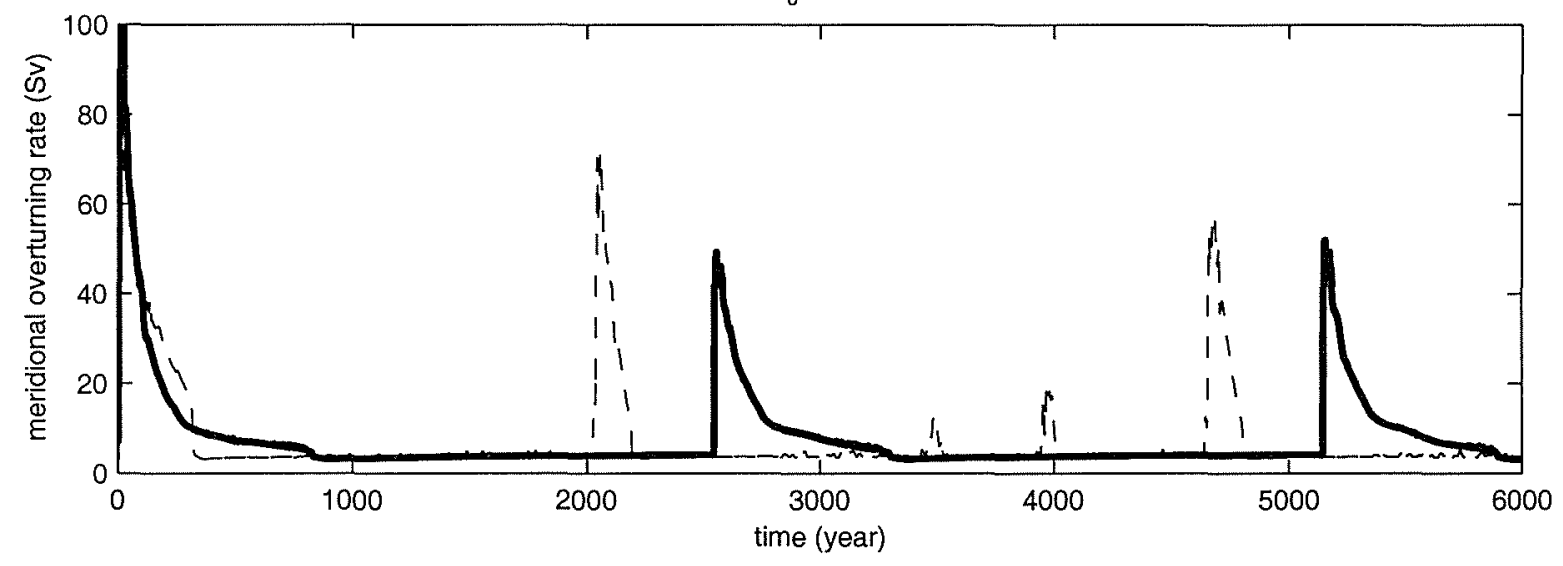

Figure 5.8: The time series of the meridional overturning rate with (a) $W_{0}=$ $0.25 \mathrm{~m}_{\text {year }}{ }^{-1}$ and (b) $W_{0}=0.3 \mathrm{~m}_{\text {year }}{ }^{-1}$. The heavy solid line represents the result for experiment CDD and the thin broken line for DDP. 
Distribution of $R_{\rho}, K_{S}$ in run DDP $\left(W_{0}=0.2 \mathrm{~m} \mathrm{year}^{-1}\right)$

With $W_{0}=0.2$ year $^{-1}$, the basin averaged $K_{T}, K_{S}$ in run DDP are:

$$
\bar{K}_{T}=0.575 \mathrm{~cm}^{2} \mathrm{~s}^{-1}, \quad \bar{K}_{S}=0.674 \mathrm{~cm}^{2} \mathrm{~s}^{-1} .
$$

Again we have $\bar{K}_{S}>\bar{K}_{T}$, indicating that salt fingering is still the dominant doublediffusive processes. In addition, the contribution of double diffusion to $\bar{K}_{S}$ is about $30 \%$, a significant increase compared to the case $W_{0}=0.1 \mathrm{~m}_{\text {year }}{ }^{-1}$. The intensity of the double-diffusive processes increases with larger freshwater forcing, which in turn leads to a larger percent of reduction in the magnitude of the thermohaline circulation.

The spatial variability of $R_{\rho}$ and $K_{S}$ in run DDP is plotted in Figure 5.9. We plot the top 1500 meters only, since in the deep ocean double diffusion is very weak and noisy. The density ratio becomes smaller in the main thermocline and also at the bottom of the thermocline. Similar to the case with $W_{0}=0.1$ m year $^{-1}$, the density ratio is small at the bottom of thermocline than in the shallower depths, and the western boundary is more salt fingering favorable than the interior.

We see that the intensity of double diffusion (as indicated by the density ratio) depends on the magnitude of the freshwater forcing; the density ratio decreases when the freshwater forcing increases. From Figure $3.1, K_{T}, K_{S}, K_{\rho}$, given by the parameterization for double-diffusive processes, increase substantially when the density ratio is close to 1. Thus, the impact of double diffusion on the thermohaline circulation depends highly on the strength of freshwater forcing.

However, the density ratio in the main thermocline remains high even for $W_{0}=$ $0.2 \mathrm{~m}$ year $^{-1}$, a forcing that is close to the critical value. This is in contrast to the real ocean, where much of the main thermocline of the Subtropical gyres has a relatively low density ratio (Schmitt, 1990). It remains a challenge to obtain a realistic density ratio distribution in the thermocline under mixed boundary conditions. The density ratio is relatively high for freshwater forcing below than the critical value, but the thermohaline circulation enters the haline mode if the freshwater forcing is increased. 

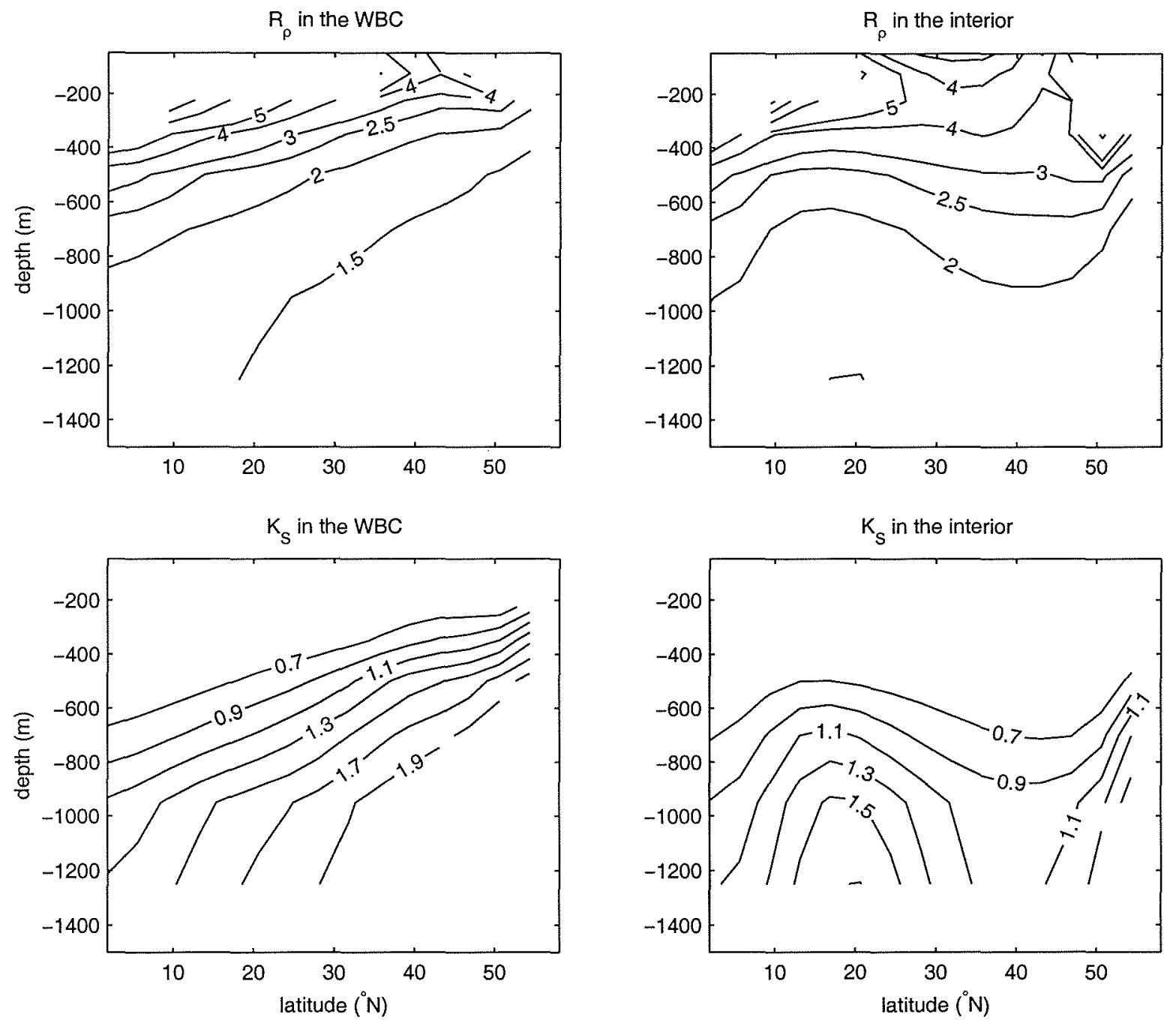

Figure 5.9: The distribution of $R_{\rho}$ (upper panels) and $K_{S}$ (lower panels) in the region of western boundary current (WBC) (left panels) and interior (right panels) respectively, in run DDP with $W_{0}=0.2 \mathrm{~m}_{\text {year }}{ }^{-1}$. Note that unequal contour intervals have been used for $R_{\rho}$, and the contour interval for $K_{S}$ is $0.2 \mathrm{~cm}^{2} \mathrm{~s}^{-1}$. 
Water Mass Structure $\left(W_{0}=0.2\right.$ mear $\left.^{-1}\right)$

Given the increased intensity of double diffusion with $W_{0}=0.2 \mathrm{~m}$ year ${ }^{-1}$ compared with $W_{0}=0.1 \mathrm{~m}_{\text {year }}{ }^{-1}$, it is not surprising that a larger difference in the water mass properties is obtained, as plotted in Figure 5.10.

Compared to run CDD, the water in run DDP becomes warmer throughout the column, but fresher in the upper 2,000 meters and saltier below. The difference is much larger than the case with $W_{0}=0.1 \mathrm{~m}_{\text {year }}{ }^{-1}$. For temperature, the increase is attributable to the intense double-diffusion in the thermocline. For salinity, there are two factors: (1) the double diffusion (mostly salt fingers) increases $K_{S}$ substantially compared to run CDD, and diffuses more salt downward. (2) since the net freshwater forcing at the surface is zero, total salt in the ocean is conserved. Increased $K_{S}$ in the main thermocline leads to the increase of salinity in the deep ocean. But since the total salt is conserved, the upper ocean has to become fresher to compensate the increased salinity in the deep ocean.

\subsubsection{Strong Freshwater Forcing}

Both runs enter the haline mode when $W_{0}=0.30 \mathrm{~m} \mathrm{year}^{-1}$ (Figure 5.8). Here we further increase the freshwater forcing to $W_{0}=0.50\left(0.80 \mathrm{~m} \mathrm{year}^{-1}\right)$ and run the model for 4,000 $(6,000)$ years. The results are plotted in Figure 5.11.

The CDD and DDP runs both show some chaotic variation over time, with characteristic "flushing", i.e., after the model stays in the haline mode for a few hundred to a few thousand years, then enters a very strong thermal mode (with the maximum meridional overturning reaching 10-70 Sv). After a relatively short period, the model enters the haline mode again.

The overturning cell in the thermal state ( at 2,100 years) and haline state (at 2,300 years) in run DDP $\left(W_{0}=0.50\right.$ m year $\left.^{-1}\right)$ are plotted in Figure 5.12. The meridional overturning cell at 2,100 years (Figure $5.12(\mathrm{a})$ ) is a very strong "thermal" cell, with a maximum of more than $40 \mathrm{~Sv}$ convected downward in the polar region. On the other hand, Figure 5.12 (b) shows the "haline" cell picture, with a dipole cell structure. The maximum evaporation minus precipitation lies at about $20^{\circ} \mathrm{N}$, and we expect the 

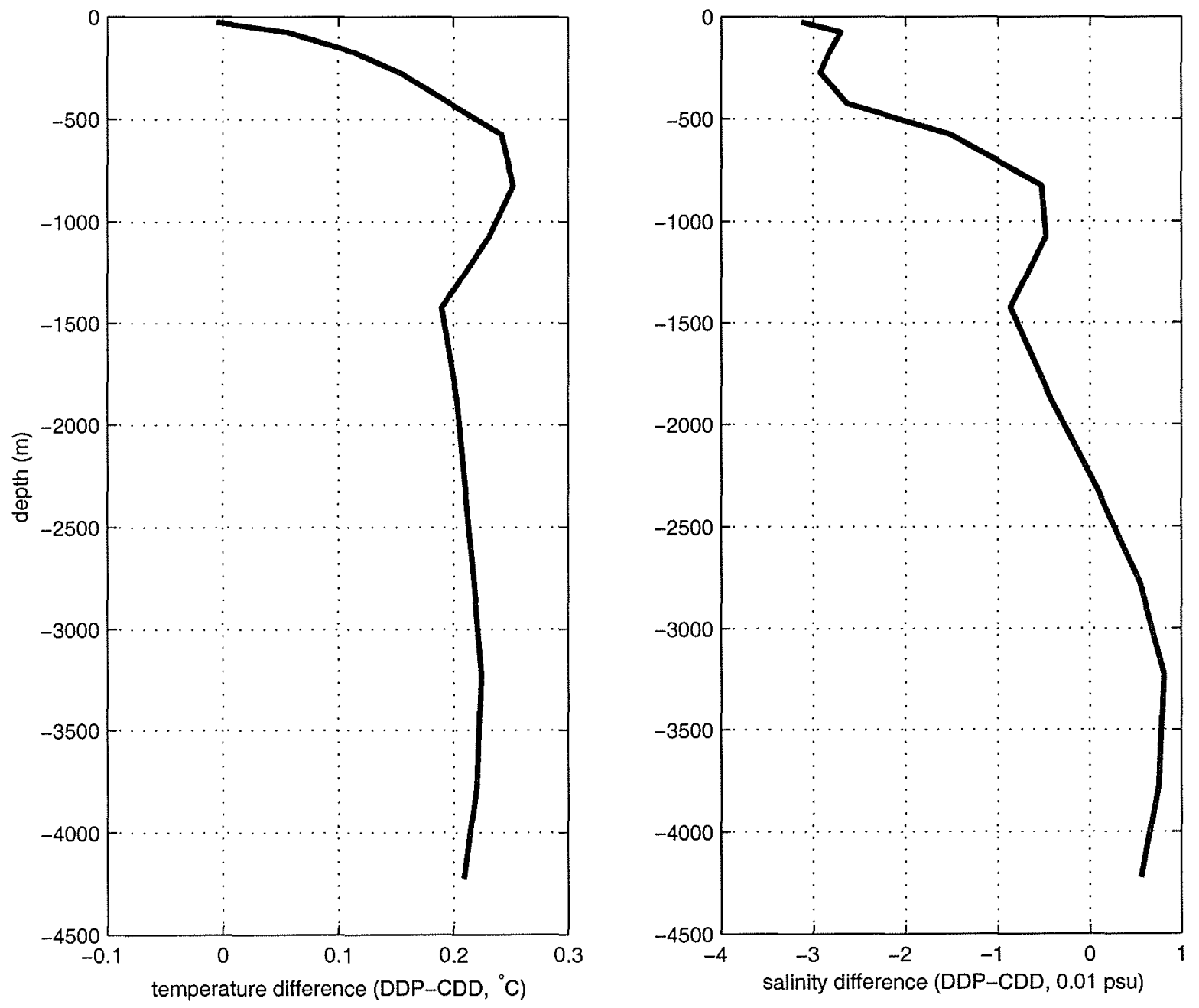

Figure 5.10: The temperature and salinity difference of run DDP from run CDD, with $W_{0}=0.2$ m year $^{-1}$. 
(a) $W_{0}=0.50 \mathrm{~m} / \mathrm{year}$

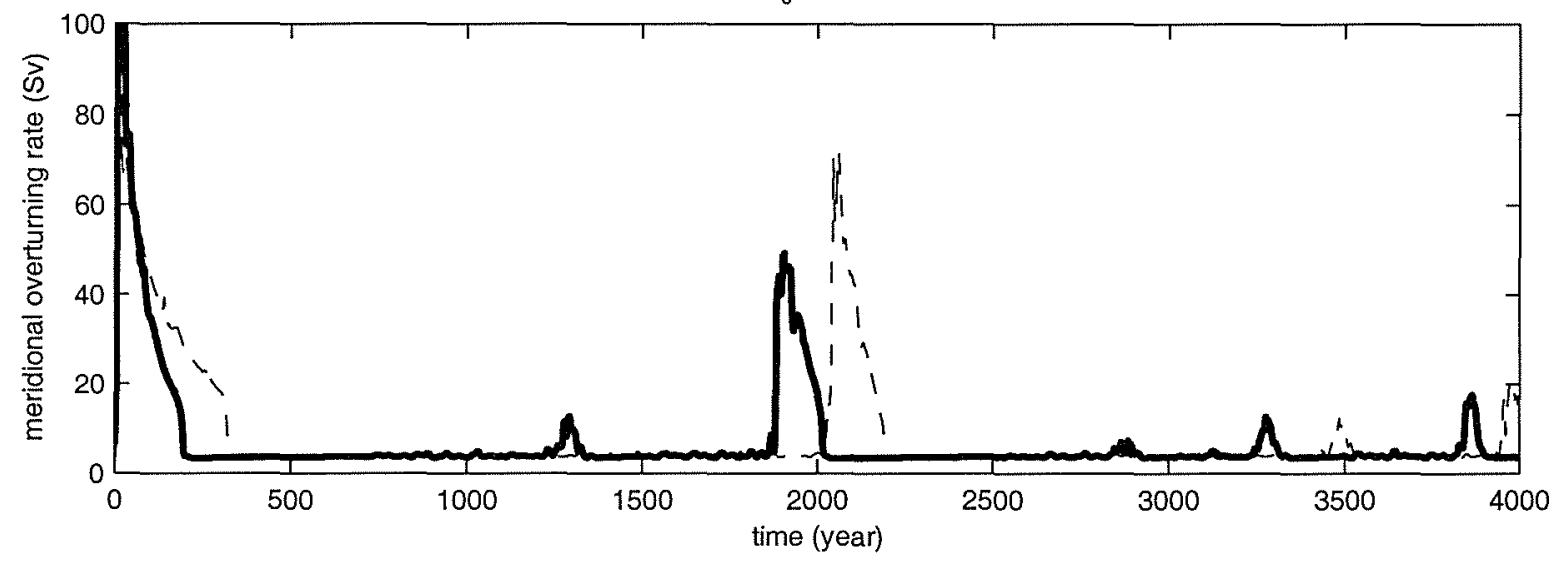

(b) $W_{0}=0.80 \mathrm{~m} /$ year

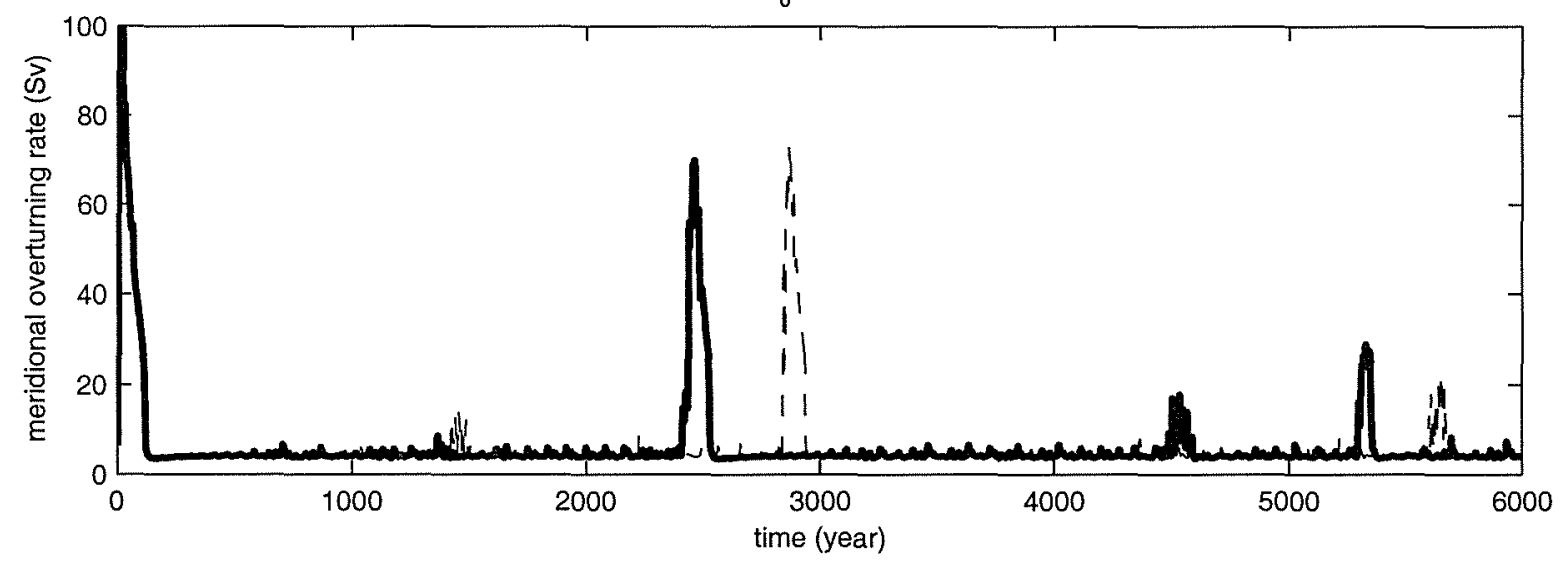

Figure 5.11: The time series of the meridional overturning rate with (a) $W_{0}=$ $0.50 \mathrm{~m}_{\text {year }}{ }^{-1}$ and (b) $W_{0}=0.80 \mathrm{~m}_{\text {year }}{ }^{-1}$. The heavy solid line represents the result for experiment CDD and the thin broken line for DDP. 
(a) meridional overturning cell at 2100 years

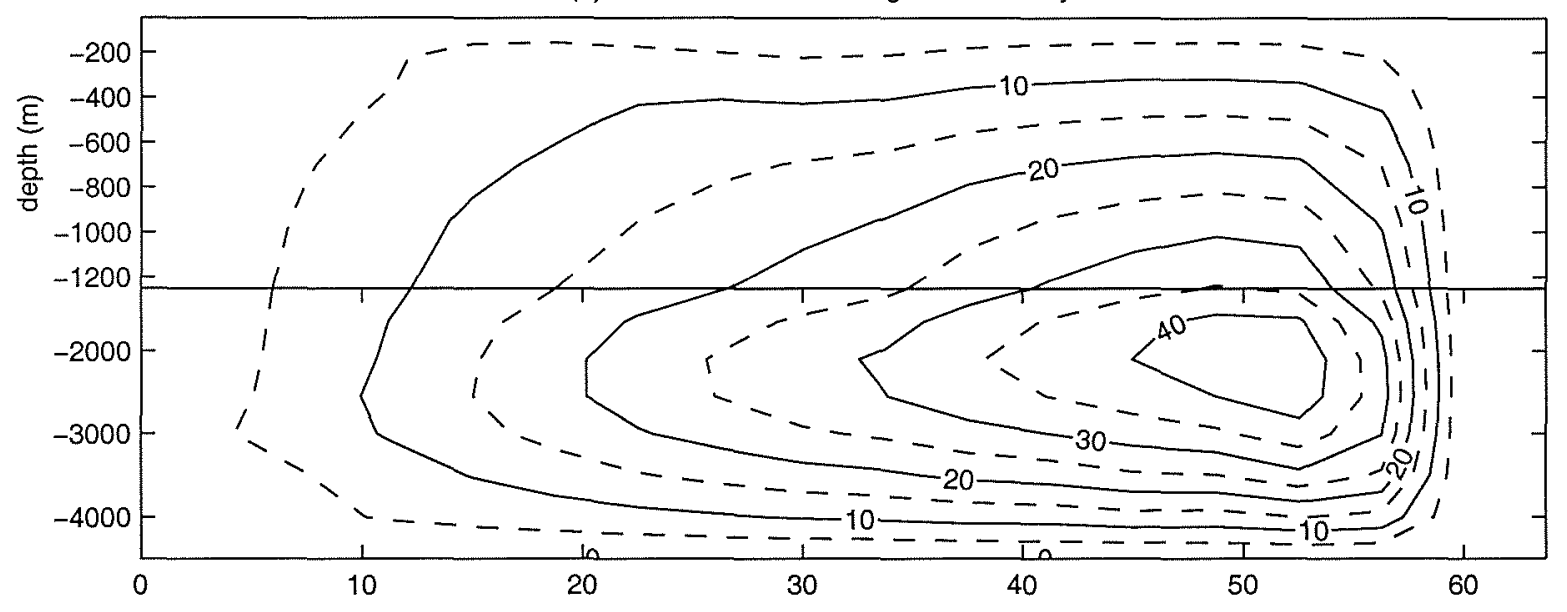

(b) meridional overturning cell at 2300 years

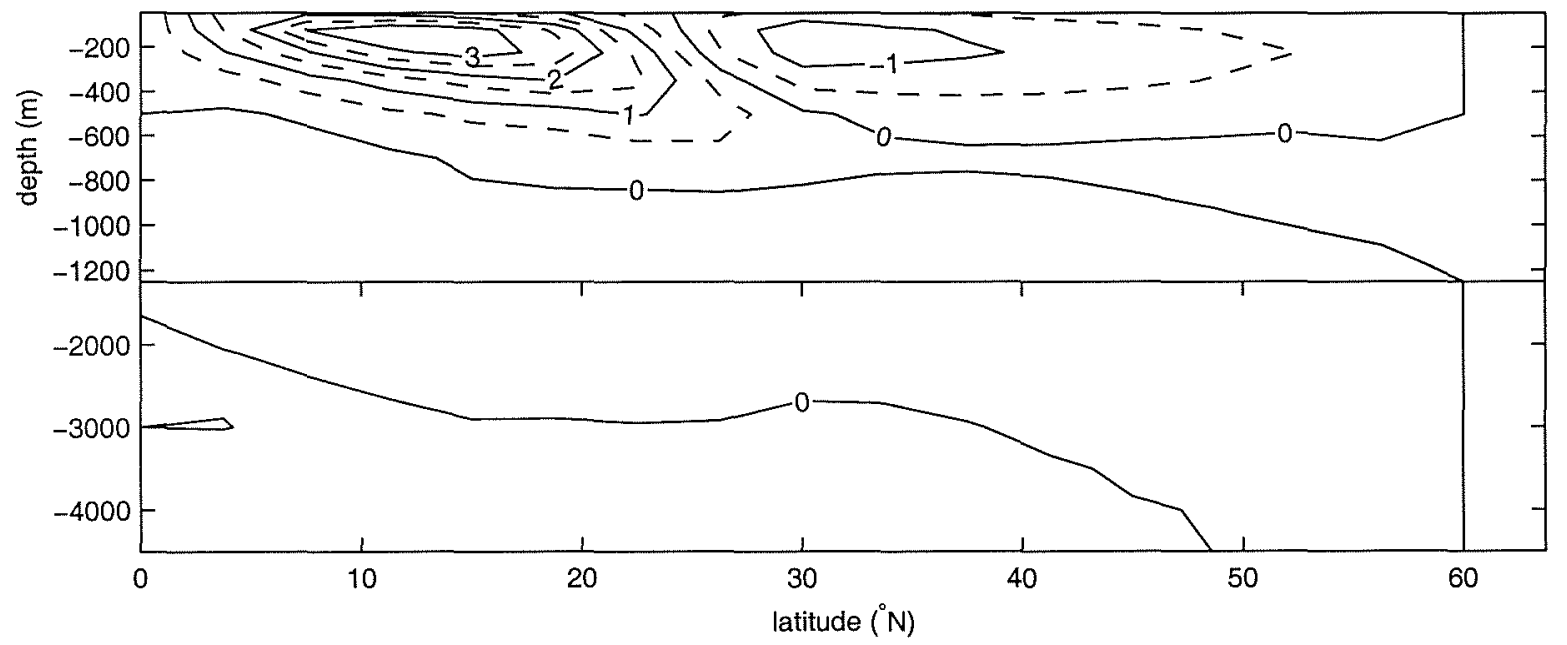

Figure 5.12: The meridional overturning cell with $W_{0}=0.50 m$ year ${ }^{-1}$ for run DDP at (a) 2,100 years and (b) 2,300 years of integration. Note that the contour interval for the upper panel is $5 \mathrm{~Sv}$ and $0.5 \mathrm{~Sv}$ for the lower panel. 
saltiest water to be formed there, and when the salt dominates the surface density difference (typical of the haline mode), the dense water is formed at $20^{\circ} \mathrm{N}$ drives convection there and forms the dipole meridional overturning cell.

The dynamics of the "haline" mode thermohaline circulation have been discussed in Marotzke (1990) and Huang (1994). When the freshwater forcing is strong enough, the convection in the polar region disappears, and the densest water is formed at lower latitudes (Figure 5.12 (b)). Convection in the "haline" mode carries large amounts of heat downward which then diffuses poleward. After hundreds or thousands of years, the deep water in the polar region becomes so warm that the water column becomes unstable, and very strong convection occurs there (Figure 5.12 (a)). The powerful convection and circulation efficiently releases the heat accumulated by the haline mode to the atmosphere, filling the deeps with cold water again. The model enters the low latitude convection state again, and this process repeats itself regularly or chaotically on a long time scale, as depicted in Figure 5.11.

\subsection{Summary}

Under mixed boundary conditions, where SST temperature is relaxed to prescribed values and a virtual salt flux is used for salinity, quasi-equilibrium experiments (with changing freshwater forcing) and individual experiments (with fixed freshwater forcing) are performed to investigate the impacts of double-diffusive processes on the stability and variability of the thermohaline circulation.

Quasi-Equilibrium experiments show that implementation of a double-diffusive parameterization alters the critical freshwater flux that destabilizes the stable thermal mode becomes smaller, consistent with the scaling analysis in Chapter 3. For weak freshwater forcing regimes, the magnitude of the thermohaline circulation is nearly identical for both run CDD and run DDP. Only when the freshwater forcing is close to the critical value, does the magnitude of the thermohaline circulation in run DDP significantly smaller than in run CDD.

Individual experiments explore some of the freshwater forcing regimes in the quasiequilibrium experiments. In the weak freshwater forcing regime $\left(W_{0}=0.1 \mathrm{~m} \mathrm{year}^{-1}\right)$, 
the magnitude and structure of the thermohaline circulation in run DDP and CDD are very similar. The distribution of density ratio in run DDP showed that the conditions for salt fingers in the main thermocline are very weak, but much stronger at the bottom of thermocline. Also, similar to the runs with the relaxation boundary conditions, western boundary regimes are more salt fingering favorable than the interior. In run DDP, the temperature and salinity become smaller in the main thermocline but larger below the thermocline, compared to run CDD. When the freshwater forcing increases to $W_{0}=0.2 \mathrm{~m}$ year $^{-1}$, a forcing in an "intermediate" regime, impact of double diffusion becomes much larger. The magnitude of the thermohaline circulation is reduced in run DDP, and the western boundary and the bottom of the main thermocline are the most double-diffusively favorable places. When $W_{0}=0.25 \mathrm{~m}_{\text {year }}{ }^{-1}$, run CDD remains in the thermal mode while run DDP enters the "haline" mode. For $W_{0}=0.30$ m year $^{-1}$, both run CDD and DDP enter the "haline" mode. When we increase the freshwater to even larger values $W_{0}=0.50,0.80 \mathrm{~m}_{\text {year }}{ }^{-1}$, the model shows the characteristics of "haline" mode circulation: after the model stays in the sluggish circulation (with convection at low latitudes) for a few hundred to a few thousand years, there will be a very strong circulation driven by convection in the polar region for several decades.

Under mixed boundary conditions, the strength of freshwater forcing, as well as the double-diffusive processes, are both crucial for the stability and variability of the thermohaline circulation. 


\section{Chapter 6}

\section{Conclusions}

\subsection{Summary}

In this thesis, the effects of diapycnal diffusivity and freshwater forcing on the thermohaline circulation are investigated through scaling analysis and numerical models. Parameterizations of double-diffusive mixing processes are discussed and implemented in the GFDL MOM2 code, and the interaction with the thermohaline circulation and underlying dynamics are investigated.

Scaling analysis provides insights to the thermohaline circulation under three types of upper ocean boundary conditions. Under "relaxation" boundary conditions and "flux" boundary conditions, there is only one equilibrium. The magnitude of the meridional overturning cell varies as the $2 / 3$ and $1 / 2$ power of the diapycnal diffusivity respectively. Under "mixed" boundary conditions, multiple equilibria are possible. The circulation states under mixed boundary conditions can be distinguished by a simple relation between the diapycnal diffusivity and the hydrologic forcing for a given thermal forcing. When $K>C E^{3 / 2}$, there are two thermal modes and one haline mode. When $K<C E^{3 / 2}$, there is only one haline mode. In addition, through stability analysis, we find that of the two thermal modes arising when $K>C E^{3 / 2}$, only one is a stable solution. The haline mode solution is always stable. Numerical experiments are implemented to test the above scaling arguments. Consistent results have been 
obtained under these three classes of boundary conditions. The relationships derived here focus attention on the need to better understand both the diapycnal mixing in the ocean and the strength of the hydrologic forcing at its surface.

The double-diffusive processes are parameterized with a scheme in which the diapycnal eddy diffusivities for heat and salt are different and depend on the local density ratio, $R_{\rho}=\alpha T_{z} / \beta S_{z}$. A background diffusivity is also applied to represent turbulent mixing in the stratified environment. The parameterization has three distinct features compared with the conventional constant eddy diffusivity assumption: (a) The vertical eddy diffusivities of temperature $\left(K_{T}\right)$ and salinity $\left(K_{S}\right)$ are different. For salt fingering mixing, $K_{S}>K_{T}$, and vice versa for diffusive layering. (b) Both $K_{T}$ and $K_{S}$ increase when the local density ratio $R_{\rho}=\alpha T_{z} / \beta S_{z}$ is close to 1 , and there is a cut off in the $R_{\rho}$ dependence. (c) A constant diapycnal diffusivity applies for the non-double-diffusive turbulent mixing. When $R_{\rho}>3.0$ or $R_{\rho}<0.3$, the diapycnal diffusivity is dominated by turbulent mixing rather than double diffusion. (d) When the vertical temperature gradient becomes less than a critical value, no double diffusion is applied. This has the effect of eliminating double diffusion in the deep ocean, where there is little evidence for its importance. The implementation of this double-diffusive parameterization leads in general to a decrease in the downward diffusion of buoyancy.

With the numerical model forced by relaxation boundary conditions on both temperature and salinity at the upper surface, three control experiments have been carried out, one with the double-diffusive parameterization determined by the local density ratio (DDP), one with constant but different diffusivities for heat and salt (GHD), and the other with a constant diapycnal eddy diffusivity for both tracers (CDD). The meridional overturning and maximum poleward heat transport in run DDP are $22 \%$ and $8 \%$ less than run CDD respectively, and only $10 \%$ and $4 \%$ decrease respectively comparing run GHD to run CDD. The temperature and salinity in run DDP and GHD are higher than in run CDD, with the change in run DDP more than twice that in run GHD. In addition, in DDP and GHD, the density ratio distribution becomes closer to unity than in run CDD, and the change in run DDP is greater than in run GHD. Interestingly, the double diffusion is stronger in the western boundary current regions than the interior, implying a close relationship between vertical shear and double-diffusive activity. Sensitivity experiments show how the variables in the pa- 
rameterization can change the relative amount of mixing due to double diffusion and background diffusion, and how this affects the thermohaline circulation. The limit on the vertical gradient of temperature operates much more in the deep ocean than in the thermocline, and the thermohaline circulation remains almost unchanged since gradients are weak at depth. Also, we found that the parameterization proposed in Chapter 3 is not very sensitive to the vertical resolution. Recent observational data suggests that the parameterization proposed for these experiments is a reasonable approximation (St. Laurent and Schmitt, 1998).

Under mixed boundary conditions, quasi-quilibrium experiments with varying freshwater forcing and individual experiments with fixed freshwater forcing are investigated. The numerical experiments are consistent with the scaling analysis: with the introduction of double diffusion, the critical freshwater forcing for the existence of the stable thermal mode becomes smaller, and the extent of reduction depends on the variables in the parameterization of double-diffusive processes. The impact of double diffusion on the thermohaline circulation increases with freshwater forcing before the critical value is reached. Individual experiments indicate that the western boundary region and the bottom of main thermocline are the places most favorable for double diffusion.

\subsection{Future Work}

Due to the limited computer resources and a desire to isolate the effects of double diffusion, the model in this thesis is limited to a one-hemisphere, flat-bottom sector. The role of double diffusion could be more throughly explored in a global model with topography, realistic coastline, a seasonal cycle, and forced and initiated from the most updated climatology. In a global model, the sensitivity of the world ocean circulation to double-diffusive processes could be tested and examined.

Another very interesting problem is the structure and tightness of the T-S relation in the main thermocline. From ventilated thermocline theory, the T-S properties can be traced back to the ocean surface, where water in contact with the atmosphere changes its properties before being subducted into the thermocline. The complicated

dynamics of subduction allows water in late winter to enter the main thermocline, 
and we would expect the observed sea surface variability in $\mathrm{T}$ and $\mathrm{S}$ to be present in the subducted water. However, this variability is barely observed in the real ocean. Double diffusion, with the diffusivity depending on the local density ratio, should be very effective in eliminating T-S anomalies, compared to the conventional constant diffusivity. This mechanism may be responsible for the tight T-S relation in the main thermocline. This issue can be addressed in a high resolution model with the inclusion of wind forcing under mixed boundary conditions, by imposing perturbations at the sea surface and then testing how double diffusion affects the T-S structure of the subducted water. 


\section{Appendix A}

\section{Solution of the equation}

$$
R^{4}=F|R-1|
$$

Here we solve the above equation in the form of (2.56) and (2.57) respectively, i.e., to get the thermal mode(s) and haline mode(s) solution respectively. To simplify the solution, we introduce the following new parameters,

$$
\begin{aligned}
x & =\frac{1}{R}, \\
F_{r} & =\frac{1}{F}
\end{aligned}
$$

then the above equation becomes

$$
\left|x^{4}-x^{3}\right|=F_{r}
$$

when $x<1$, we obtain a thermal mode and when $x>1$ we have a haline mode.

\section{A.1 Thermal Mode(s) Solution}

The fourth-order equation

$$
x^{4}-x^{3}+F_{r}=0
$$


can be reduced to solving the following two quadratic equations:

$$
\begin{aligned}
& x^{2}+(-1+\sqrt{8 y+1}) \frac{x}{2}+y\left(1-\frac{1}{\sqrt{8 y+1}}\right)=0 \\
& x^{2}+(-1-\sqrt{8 y+1}) \frac{x}{2}+y\left(1+\frac{1}{\sqrt{8 y+1}}\right)=0 .
\end{aligned}
$$

where $y$ is the arbitrary root of the following cubic equation:

$$
y^{3}-F_{r} y-\frac{F_{r}}{8}=0
$$

The two criteria for equation $(A .5)$ and equation $(A .6)$ are

$$
\begin{aligned}
\Delta_{T H 1} & =\frac{1}{2}-2 y-\frac{1}{2 \sqrt{8 y+1}}, \\
\Delta_{T H 2} & =\frac{1}{2}-2 y+\frac{1}{2 \sqrt{8 y+1}}
\end{aligned}
$$

respectively, which determines when there are real roots for equation $(A .5)$ and equation $(A .6)$.

In order for $x$ to be a real root, we take the real root of the above cubic equation, which is

$$
y=\sqrt[3]{F_{r} / 16+\sqrt{\Delta_{T}}}+\sqrt[3]{F_{r} / 16-\sqrt{\Delta_{T}}}
$$

where

$$
\begin{aligned}
\Delta_{T} & =\frac{F_{r}^{2}}{256}-\frac{F_{r}^{3}}{27} \\
& =\frac{F_{r}^{2}}{27}\left(\frac{27}{256}-F_{r}\right)
\end{aligned}
$$

Next we discuss the solutions according to the sign of $\Delta_{T}$.

\section{$\underline{\underline{\Delta_{T}>0}}$}

When $\Delta_{T}>0$, i.e.,

$$
F_{r}<27 / 256
$$

$\sqrt{\Delta_{T}}$ is real, then

$$
y=\frac{\left(F_{r} / 16+\sqrt{\Delta_{T}}\right)+\left(F_{r} / 16-\sqrt{\Delta_{T}}\right)}{\sqrt[3]{\left(F_{r} / 16+\sqrt{\Delta_{T}}\right)^{2}}+\sqrt[3]{\left(F_{r} / 16-\sqrt{\Delta_{T}}\right)^{2}}-\sqrt[3]{\left(F_{r} / 16\right)^{2}-\Delta_{T}}}
$$




$$
\begin{aligned}
& =\frac{F_{r} / 8}{\sqrt[3]{\left(F_{r} / 16+\sqrt{\Delta_{T}}\right)^{2}}+\sqrt[3]{\left(F_{r} / 16-\sqrt{\Delta_{T}}\right)^{2}}-F_{r} / 3} \\
& <\frac{F_{r} / 8}{2 F_{r} / 3-F_{r} / 3}=\frac{3}{8}
\end{aligned}
$$

where the unequal equation

$$
\begin{aligned}
\sqrt[3]{\left(F_{r} / 16+\sqrt{\Delta_{T}}\right)^{2}}+\sqrt[3]{\left(F_{r} / 16-\sqrt{\Delta_{T}}\right)^{2}} & >2 \sqrt[3]{\left(F_{r} / 16+\sqrt{\Delta_{T}}\right)\left(F_{r} / 16-\sqrt{\Delta_{T}}\right)} \\
& =\frac{2 F_{r}}{3}
\end{aligned}
$$

has been used.

With

$$
y<\frac{3}{8}
$$

it is straitforward to show that

$$
\frac{1}{2 \sqrt{8 y+1}}>\left|\frac{1}{2}-2 y\right| .
$$

Thus we have

$$
\begin{aligned}
& \Delta_{T H 1}<0 \\
& \Delta_{T H 2}>0
\end{aligned}
$$

i.e, there is no real root for equation (A.5) and two real roots for equation (A.6). The two real roots can be written as

$$
x_{T H}^{1,2}=\frac{1+\sqrt{8 y+1} \pm \sqrt{4 \Delta_{T H 2}}}{4}
$$

Now we check whether the above two roots satisfy thermal modes condition $x<1$. First of all,

$$
\begin{aligned}
x_{T H}^{2} & =\frac{1+\sqrt{8 y+1}-\sqrt{4 \Delta_{T H 2}}}{4} \\
& <\frac{1+\sqrt{8 y+1}}{4}<\frac{3}{4}
\end{aligned}
$$

and it is a thermal mode solution. In addition, the solution $x_{T H}^{1}$ is a singular function of $y$, for $0<y<3 / 8$, we have

$$
\frac{3}{4}<x_{T H}^{1}<1
$$


which is also a possible thermal mode.

\section{$\underline{\underline{\Delta_{T}<0}}$}

When $\Delta_{T}<0$, i.e.,

$$
F_{r}>27 / 256
$$

$\sqrt{\Delta_{T}}$ is imaginary. Write

$$
\sqrt{\Delta_{T}}=i b
$$

where

$$
b=\sqrt{F_{r}^{3} / 27-F_{r}^{2} / 256} .
$$

Then

$$
\begin{aligned}
y & =\sqrt[3]{F_{r} / 16+i b}+\sqrt[3]{F_{r} / 16-i b} \\
& =2 A
\end{aligned}
$$

where $A$ is the real part of $\sqrt[3]{F_{r} / 16+i b}$.

Since

$$
F_{r} / 16+i b=r e^{i \theta}
$$

where

$$
\begin{aligned}
r^{2} & =\left(F_{r} / 16\right)^{2}+b^{2}=F_{r}^{3} / 27, \\
\cos \theta & =F_{r} / 16 r=\frac{9}{16 \sqrt{3 F_{r}}} .
\end{aligned}
$$

Then we have

$$
y=2 \sqrt[3]{r} \cos \frac{\theta}{3}=\frac{2 \sqrt{3 F_{r}}}{3} \cos \frac{\theta}{3}
$$

Given the condition (A.22), and combining with (A.28),we have

$$
0<\cos \theta<1
$$

i.e.,

$$
|\theta|<\frac{\pi}{2}
$$


thus we obtain

$$
\cos \frac{\theta}{3}>\cos \theta
$$

As a result, we have

$$
\begin{aligned}
y & =\frac{2 \sqrt{3 F_{r}}}{3} \cos \frac{\theta}{3} \\
& >\frac{2 \sqrt{3 F_{r}}}{3} \cos \theta \\
& =\frac{2 \sqrt{3 F_{r}}}{3} \frac{9}{16 \sqrt{3 F_{r}}} \\
& =\frac{3}{8} .
\end{aligned}
$$

Then

$$
\begin{aligned}
\Delta_{T H 2} & =\frac{1}{2}-2 y+\frac{1}{2 \sqrt{8 y+1}} \\
& <\frac{1}{2}-2 \times \frac{3}{8}+\frac{1}{2 \times 2} \\
& =0 .
\end{aligned}
$$

Since

$$
\Delta_{T H 1}<\Delta_{T H 2}
$$

Therefore both $\Delta_{T H 1}$ and $\Delta_{T H 2}$ are negative, and there is no real solution for equation (2.44) when condition $(A .22)$ is satisfied.

$\underline{\underline{\Delta_{T}}=0}$

When $\Delta_{T}=0$, i.e., $F_{r}=27 / 256$, the two thermal mode solutions are equal, i.e, we have:

$$
x_{T H}^{1}=x_{T H}^{2}=\frac{3}{4}
$$




\section{A.2 Haline Mode(s) Solution}

The fourth-order equation

$$
x^{4}-x^{3}-F_{r}=0
$$

can also be reduced to the form of equation $(A .5)$ and $(A .6)$, while $y$ is determined by a new cubic equation

$$
y^{3}+F_{r} y+\frac{F_{r}}{8}=0
$$

One of the roots of the above cubic equation is

$$
\begin{aligned}
y & =\sqrt[3]{-F_{r} / 16+\sqrt{\Delta_{S}}}+\sqrt[3]{-F_{r} / 16-\sqrt{\Delta_{S}}} \\
& =\sqrt[3]{\sqrt{\Delta_{S}}-F_{r} / 16}-\sqrt[3]{\sqrt{\Delta_{S}}+F_{r} / 16}
\end{aligned}
$$

where

$$
\Delta_{S}=F_{r}^{2} / 256+F_{r}^{3} / 27>0
$$

Thus it is obvious that $y<0$. On the other hand,

$$
\begin{aligned}
y & =\frac{\left(\sqrt{\Delta_{S}}-F_{r} / 16\right)-\left(F_{r} / 16+\sqrt{\Delta_{S}}\right)}{\sqrt[3]{\left(\sqrt{\Delta_{S}}-F_{r} / 16\right)^{2}}+\sqrt[3]{\left(\sqrt{\Delta_{S}}+F_{r} / 16\right)^{2}}+\sqrt[3]{\Delta_{S}-\left(F_{r} / 16\right)^{2}}} \\
& >-\frac{1}{8}
\end{aligned}
$$

For $-1 / 8<y<0$, the inequality $(A .16)$ still holds. Thus we have

$$
\begin{gathered}
\Delta_{H A 1}<0 \\
\Delta_{H A 2}>0 .
\end{gathered}
$$

Therefore only the second quadratic equation has real roots

$$
x_{H A}^{1,2}=\frac{1+\sqrt{8 y+1} \pm \sqrt{4 \Delta_{H A 2}}}{4} .
$$

Now we check whether the haline mode requirement $x>1$ is satisfied. Since

$$
\begin{aligned}
x_{H A}^{2} & =\frac{1+\sqrt{8 y+1}-\sqrt{4 \Delta_{H A 2}}}{4} \\
& <\frac{1+\sqrt{8 y+1}}{4}<\frac{1}{2},
\end{aligned}
$$


thus $x_{H A}^{2}$ is not a haline mode solution. On the other hand, $x_{H A}^{1}$ is a singular function of $y$ and for $-1 / 8<y<0$ we have

$$
x_{H A}^{1}>1
$$

and thus it is a real haline mode solution.

In summary, we can transform the solution back to original parameters, when $F>$ $256 / 27$, there are two possible thermal modes; when $F<256 / 27$, no thermal mode is possible. Thus the criterion $F=256 / 27$ determines the existence of thermal modes. In addition, there is always one and only one haline mode solution given any external conditions. 


\section{References}

Broecker, W. S., D. M. Peteet, and D. Rind, 1985. Does the ocean-atmosphere system have more than one stable mode of operation? Nature, 315, 21-26.

Bryan, K., 1969. A numerical method for the study of the circulation of the world ocean. J. Comput. Phys., 4, 347-376.

Bryan, F., 1986. High-latitude salinity effects and interhemispheric thermohaline circulations. Nature, 323, 301-304.

Bryan, F., 1987. On the parameter sensitivity of primitive equation ocean general circulation model. J. Phys. Oceanogr., 17, 970-985.

Bryan, K. and M. Cox, 1972. An approximate equation of state for numerical models of ocean circulation. J. Phys. Oceanogr., 2, 510-514.

Cummins, P. F., G. Holloway, and A. E. Gargett, 1990. Sensitivity of the GFDL ocean general circulation model to a parameterization of vertical diffusion. $J$. Phys. Oceanogr., 20, 817-830.

Danabasoglu, G. and J. C. McWilliams, 1995. Sensitivity of the Global Ocean Circulation Model to Parameterizations of Mesoscale Tracer Transports. $J$. Climate, 8, 2967-2987.

Federov, K. N., 1988. Layer thicknesses and effective diffusivities in "diffusive" thermohaline convection in the ocean. In Nihoul, J. and B. Jamart, editors, Small-Scale Turbulence and Mixing in the Ocean, 46, pages 471-480. Elsevier.

Gargett, A. E. and B. Ferron, 1996. The effects of differential vertical diffusion of T and $\mathrm{S}$ in a box model of thermohaline circulation. J. Marine Res., 54, 827-866.

Gargett, A. E. and G. Holloway, 1992. Sensitivity of the GFDL ocean model to different diffusivities for Heat and salt. J. Phys. Oceanogr., 22, 1158-1177.

Gargett, A. E. and R. W. Schmitt, 1982. Observations of salt fingers in the central waters of the eastern North Pacific. J. Geophys. Res., 87(C10), 8017-8029.

Garrett, C. J. R. and W. H. Munk, 1972. Oceanic mixing by breaking internal waves. Deep-Sea Res., 19, 823-832.

Gent, P. R. and J. C. McWilliams, 1990. Isopycnal mixing in ocean circulation models. J. Phys. Oceanogr., 20, 150-155. 
Gent, P. R., J. Willebrand, T. J. McDougall, and J. C. McWilliams, 1995. Parameterizing eddy-induced tracer transports in ocean circulation models. J. Phys. Oceanogr., 25, 463-474.

Gerdes, R., C. Koberle, A. Beckmann, P. Herrmann, and J. Willebrand, 1998. Mechanisms for spreading of Mediterranean Water in coarse resolution numerical models. J. Phys. Oceanogr. in press.

Gregg, M. C., 1989. Scaling turbulent dissipation in the thermocline. J. Geophys. Res., 94, 9686-9698.

Gregg, M. C. and T. B. Sanford, 1987. Shear and turbulence in the thermohaline staircases. Deep-Sea Res., 34, 1689-1696.

Griffies, S. M., A. Gnanadesikan, R. C. Pacanowski, V. D. Larichev, J. K. Dukowicz, and R. D. Smith, 1998. Isoneutral diffusion in a z-coordinate ocean model. $J$. Phys. Oceanogr., 28(5), 805-830.

Hamilton, J. M., N. S. Oakey, and D. E. Kelley, 1993. Salt finger signatures in microstructure measurements. J. Geophys. Res., 98(C2), 2453-2460.

Haney, R. L., 1971. Surface Thermal Boundary Condition for Ocean Circulation Models. J. Phys. Oceanogr., 1, 241-248.

Hellerman, S. and M. Rosenstein, 1983. Normal monthly wind stress over the world ocean with error estimates. J. Phys. Oceanogr., 13, 1093-1104.

Hogg, N., P. Biscaye, W. Gardner, and W. J. Schmitz, 1982. On the transport and modification of Antarctic Bottom Water in the Vema Channel. J. Marine Res., 40(suppl.), 231-263.

Huang, R. X., 1993. Real freshwater flux as the upper boundary condition for the salinity balance and thermohaline circulation forced by evaporation and precipitation. J. Phys. Oceanogr., 23, 2428-2446.

Huang, R. X., 1994. Thermohaline circulation: Energetics and variability in a single-hemisphere basin model. J. Geophys. Res., 99(C6), 12471-12485.

Huang, R. X., 1995. Advances in Theories of Wind-Driven and Thermohaline Circulation, a lecture series. University of Hawaii at Manoa, SOEST. 213 pp.

Huang, R. X. and R. L. Chou, 1994. Parameter sensitivity study of the saline circulation. Climate Dyn., 9, 391-409.

Huang, R. X. and J. Yang, 1996. Deep-water upwelling in the frictional western boundary layer. J. Phys. Oceanogr., 26, 2243-2250. 
Huang, R. X., J. Luyten, and H. M. Stommel, 1992. Multiple equilibrium states in combined thermal and saline circulation. J. Phys. Oceanogr., 22, 231-246.

Huppert, H. E., 1971. On the stability of a series of double-diffusive layers. DeepSea Res., 18, 1005-1021.

Ingham, M. C., 1966. The salinity extrema of the world ocean. $\mathrm{PhD}$ thesis, Oregon State University.

Keigwin, L. A., G. A. Jones, S. J. Lehman, and E. A. Boyle, 1991. Deglacial meltwater discharge, North Atlantic deep circulation, and abrupt climate change. J. Geophys. Res., 96, 16811-16826.

Kelley, D. E., 1984. Effective diffusivities within oceanic thermohaline staircases. J. Geophys. Res., 89, 10484-10488.

Kelley, D. E., 1990. Fluxes through diffusive staircases: a new formulation. $J$. Geophys. Res., 95, 3365-3371.

Klinger, B. A. and J. Marotzke, 1998. Behavior of double hemisphere thermohaline flows in a single basin. J. Phys. Oceanogr. in press.

Kunze, E., 1990. The evolution of salt fingers in inertial wave shear. J. Marine Res., 48, 471-504.

Ledwell, J. R., A. J. Watson, and C. S. Law, 1993. Evidence for slow mixing across the pycnocline from an open-ocean tracer-release experiment. Nature, 364, 701-703.

Levitus, S., 1982. Climatological Atlas Of The World Ocean. NOAA Professional Paper 13. Govt. Printing Office, Washington, D.C.

Lueck, R., 1987. Microstructure measurements in a thermohaline staircase. DeepSea Res., 34, 1677-1688.

Mack, S. A., 1985. Two-dimensional measurements of ocean microstructure: The role of double diffusion. J. Phys. Oceanogr., 15, 1581-1604.

Mack, S. A., 1989. Towed chain measurement of ocean microstructure. J. Phys. Oceanogr., 19, 1108-1129.

Mack, S. A. and H. C. Schoeberlein, 1993. Discriminating salt fingering from turbulence-induced microstructure: analysis of towed temperature-conductivity chain data. J. Phys. Oceanogr., 23, 2073-2106. 
Marmorino, G. O., 1987. Observations of small-scale mixing processes in the seasonal thermocline, Pt. 1, Salt fingering. J. Phys. Oceanogr., 17(9), 1339-1347.

Marmorino, G. O., W. K. Brown, and W. D. Morris, 1987. Two-dimensional temperature structure in the C-SALT thermohaline staircase. Deep-Sea Res., 23, $1667-1675$.

Marotzke, J., 1990. Instabilities and multiple equilibria of the thermohaline circulation. $\mathrm{PhD}$ thesis, Ber.Inst.Meeresk.Kiel.

Marotzke, J., 1997. Boundary mixing and the dynamics of 3-dimensional thermohaline circulation. J. Phys. Oceanogr., 27, 1713-1728.

Marotzke, J. and B. A. Klinger, 1998. Boundary mixing and equatorially asymmetric thermohaline circulation. J. Phys. Oceanogr. submitted.

Martinson, D. G., 1990. Evolution of the Southern Ocean winter mixed layer and sea ice: open ocean deep water formation and ventilation. J. Geophys. Res., $\mathbf{9 5}, 11,641-11,654$.

McDougall, T. J., 1983. Greenland Sea Bottom Water formation: a balance between advection and double-diffusion. Deep-Sea Res., 30, 1109-1118.

McDougall, T. J. and J. R. Taylor, 1984. Flux measurements across a finger interface at low values of the stability ratio. J. Marine Res., 42, 1-14.

McDougall, T. J. and J. A. Whitehead, 1984. Estimates of the relative roles of diapycnal, isopycnal and double-diffusive mixing in Antarctic Bottom Water in the North Atlantic. J. Geophys. Res., 89, 10,479-10,483.

Muench, R. D., H. J. S. Fernando, and G. R. Stegen, 1990. Temperature and salinity Staircases in the Northwestern Weddell Sea. J. Phys. Oceanogr., 20, 295-306.

Munk, W. H., 1966. Abyssal recipes. Deep-Sea Res., 13, 707-730.

Munk, W. H. and C. Wunsch, 1998. The moon and mixing: abyssal recipes II. Deep-Sea Res. submitted.

Neal, V. T., S. Neshyba, and W. Denner, 1969. Thermal stratification in the Arctic Ocean. Science, 166, 373-374.

Neshyba, S., V. T. Neal, and W. W. Denner, 1971. Temperature and conductivity measurements under Ice Island T-3. J. Geophys. Res., 76, 8107-8120. 
Pacanowski, R. C., 1995. MOM2 Documentation, User's Guide and Reference Manual. Technical Report 3, GFDL Ocean Group Technical Report.

Pacanowski, R., K. Dixon, and A. Rosati, 1993. The G.F.D.L Modular Ocean Model Users Guide. Technical Report 2, GFDL Ocean Group Technical Report.

Padman, L. and T. M. Dillon, 1987. Vertical fluxes through the Beaufort sea thermohaline staircase. J. Geophys. Res., 92, 10,799-10,806.

Padman, L. and T. M. Dillon, 1988. On the horizontal extent of the Canada Basin thermohaline steps. J. Phys. Oceanogr., 18, 1458-1462.

Park, Y.-G. and K. Bryan, 1998. Intercomparison of thermally driven circulations from a depth coordinate model and an isopycnal layer model: Part I. A scaling law - sensitivity to vertical diffusivity. J. Phys. Oceanogr. submitted.

Polzin, K., 1996. Statistics of the Richardson Number: Mixing models and finestructure. J. Phys. Oceanogr., 26, 1409-1425.

Polzin, K., J. M. Toole, and R. W. Schmitt, 1995. Finescale parameterizations of turbulent dissipation. J. Phys. Oceanogr., 25, 306-328.

Polzin, K. L., J. M. Toole, J. R. Ledwell, and R. W. Schmitt, 1997. Spatial variability of turbulent mixing in the abyssal ocean. Science, 276, 93-96.

Rahmstorf, S., 1995. Bifurcations of the Atlantic thermohaline circulation in response to changes in the hydrological cycle. Nature, 378, 145-149.

Redi, M. H., 1982. Oceanic isopycnal mixing by coordinate rotation. J. Phys. Oceanogr., 12, 1154-1158.

Robertson, R., L. Padman, and M. D. Levine, 1995. Finestructure, microstructure, and vertical mixing processes in the upper ocean in the western Weddel Sea. $J$. Geophys. Res., 100, 18,517-18,535.

Ruddick, B., 1983. A practical indicator of the stability of the water column to double-diffusive activity. Deep-Sea Res., 30, 1105-1107.

Ruddick, B., 1992. Intrusive mixing in a Mediterranean salt lens: intrusion slopes and dynamical mechanisms. J. Phys. Oceanogr., 22, 1274-1285.

Ruddick, B. and J. S. Turner, 1979. The vertical length scale of double-diffusive intrusions. Deep-Sea Res., 26A, 23-40.

Ruddick, B. and L. Zhang, 1989. The mythical thermohaline oscillator? J. Marine Res., 47, 717-746. 
Schmitt, R. W., 1979. The growthrate of super-critical salt fingers. Deep-Sea Res., $26 \mathrm{~A}, 23-40$.

Schmitt, R. W., 1981. Form of temperature-salinity relationship in the central water: evidence for double-diffusive mixing. J. Phys. Oceanogr., 11, 10151026.

Schmitt, R. W., 1988. Mixing in a thermohaline staircase. In Nihoul, J. and B. Jamart, editors, Small-Scale Turbulence and Mixing in the ocean, volume 46 of Elsevier Oceanogr. Ser., pages 435-452. Elsevier, New York.

Schmitt, R. W., 1990. On the density ratio balance in the Central Water. J. Phys. Oceanogr., 20, 900-906.

Schmitt, R. W., 1994. Double diffusion in oceanography. Annu. Rev. Fluid Mech., 26, 255-85.

Schmitt, R. W., 1995. The ocean component of the global water cycle. Rev. Geophys., 33 (supplement, pt 2), 1395-1409. U.S National Report to International Union of Geodesy and Geophysics.

Schmitt, R. W., H. Perkins, J. D. Boyd, and M. C. Stalcup, 1987. C-SALT:an investigation of the thermohaline staircase in the western tropical North Atlantic. Deep-Sea Res., 34, 1697-1704.

Schmitt, R. W., P. S. Bogden, and C. E. Dorman, 1989. Evaporation minus precipitation and density fluxes for the North Atlantic. J. Phys. Oceanogr., 19, $1208-1221$.

Schmitz, W. J., J. R. Luyten, and R. W. Schmitt, 1993. On the Florida current T/S Envelope. Bulletin of Marine Science, 53, 1048-1065.

Shen, C. and R. W. Schmitt, 1996. The wavenumber spectrum of salt fingers. In Brandt, A. and H. Fernando, editors, Double-Diffusive Convection, volume 94, pages $305-312$.

Shirtcliffe, T. G. L., 1967. Thermosolutal convection: observation of an overstable mode. Nature, 213, 489-490.

Spall, M. A., 1998. A simple model of the large scale circulation of Mediterranean Water and Labrador Sea Water. Deep-Sea Res. in press.

St. Laurent, L. and R. W. Schmitt, 1998. The contribution of salt fingers to vertical mixing in the North Atlantic tracer release experiment. J. Phys. Oceanogr. in press. 
Stern, M. E., 1960. The "salt fountain" and thermohaline convection. Tellus, 12, $172-175$.

Stern, M. E., 1975. Ocean Circulation Physics. Academic Press.

Stommel, H. M., 1961. Thermohaline convection with two stable regimes of flow. Tellus, 13, 224-230.

Stommel, H. and A. B. Arons, 1960. On the abyssal circulation of the world ocean. Part II: An idealized model of the circulation pattern and amplitude in oceanic basins. Deep-Sea Res., 6, 339-343.

Taylor, J., 1991. Laboratory experiments on the formation of salt fingers after the decay of turbulence. J. Geophys. Res., 96(C7), 12,497-12,510.

Toole, J. M., K. L. Polzin, and R. W. Schmitt, 1994. Estimates of diapycnal mixing in the abyssal ocean. Science, 264, 1120-1123.

Turner, J. S., 1973. Buoyancy Effects in Fluids. Cambridge University Press, Cambridge. 367 pp.

Turner, J. S., 1978. Double-diffusive intrusions into a density gradient. J. Geophys. Res., 83, 2887-2901.

Warren, B. A., 1983. Why is no deep water formed in the North Pacific? J. Marine Res., 41, 327-347.

Weaver, A. J. and Hughes T. M. C., 1992. Stability and variability of the thermohaline circulation and its link to climate. Trends Phys. Oceanogr., 1, 15-70.

Weaver, A. J. and E. S. Sarachik, 1990. On the importance of vertical resolution in certain ocean general circulation models. J. Phys. Oceanogr., 20, 600-609.

Weaver, A. J., J. Marotzke, P. F. Cummins, and E. S. Sarachik, 1993. Stability and variability of the thermohaline circulation. J. Phys. Oceanogr., 23, 39-60.

Weber, S. L., 1998. Parameter sensitivity of a coupled atmosphere-ocean model. Clim. Dyn., 14, 201-212.

Welander, P., 1971. The thermocline problem. Phil. Trans. Roy. Soc. Lond. A., 270, 415-421.

Welander, P., 1986. Thermohaline effects in the ocean circulation and related simple models. In Anderson, D. L. T. and J. Willebrand, editors, Large-Scale Transport Processes in Oceans and Atmosphere, NATO ASI series. Reidel. 
Whitehead, J. A., 1995. Thermohaline ocean processes and models. Annu. Rev. Fluid Mech., 27, 89-113.

Wright, D. and T. F. Stocker, 1992. Sensitivity of a zonally averaged global ocean circulation model. J. Geophys. Res., 97(C8), 12,707-12,730.

Yin, F. L. and I. Y. Fung, 1991. Net Diffusivity in Ocean General Circulation Models With Nonuniform Grids. J. Geophys. Res., 96, 10773-10776.

Zhang, J., R. W. Schmitt, and R. X. Huang, 1998a. Sensitivity of GFDL Modular Ocean Model to the parameterization of double-diffusive processes. J. Phys. Oceanogr., 28, 589-605.

Zhang, J., R. W. Schmitt, and R. X. Huang, 1998b. The relative influence of diapycnal mixing and hydrologic forcing on the stability of the thermohaline circulation. J. Phys. Oceanogr. in press. 